University of Louisville

ThinkIR: The University of Louisville's Institutional Repository

Electronic Theses and Dissertations

$5-2010$

\title{
Chitosan nanoparticles to encapsulate a biofilm degrading enzyme for treatment of CRBSI.
}

Lindsay Nicole Strotman

University of Louisville

Follow this and additional works at: https://ir.library.louisville.edu/etd

\section{Recommended Citation}

Strotman, Lindsay Nicole, "Chitosan nanoparticles to encapsulate a biofilm degrading enzyme for treatment of CRBSI." (2010). Electronic Theses and Dissertations. Paper 1395.

https://doi.org/10.18297/etd/1395

This Master's Thesis is brought to you for free and open access by ThinkIR: The University of Louisville's Institutional Repository. It has been accepted for inclusion in Electronic Theses and Dissertations by an authorized administrator of ThinkIR: The University of Louisville's Institutional Repository. This title appears here courtesy of the author, who has retained all other copyrights. For more information, please contact thinkir@louisville.edu. 


\title{
CHITOSAN NANOPARTICLES TO ENCAPSULATE A BIOFILM DEGRADING ENZYME FOR TREATMENT OF CRBSI
}

\author{
By
}

Lindsay Nicole Strotman

B.S., University of Louisville, 2009

\author{
A Thesis \\ Submitted to the Faculty of the \\ University of Louisville \\ J.B. Speed School of Engineering \\ as Partial Fulfillment of the Requirements \\ for the Professional Degree
}

MASTER OF ENGINEERING

Department of Bioengineering

May 2010 



\section{CHITOSAN NANOPARTICLES TO ENCAPSULATE A BIOFILM DEGRADING ENZYME FOR TREATMENT OF CRBSI}

Submitted by:

Lindsay Nicole Strotman

A Thesis Approved on

$\frac{\text { May } 6,2010}{\text { (May } 6,2010)}$

by the Following Reading and Examination Committee:

Andrea S. Gobin, Thesis Director

André M. Gobin

Gerold A. Willing 


\section{ACKNOWLEDGEMENTS}

I would like to start out with perhaps the most profound person who has not only guided me throughout my thesis but also my career development. That person is my thesis director Andrea Gobin, PhD. Without her constant guidance, support and sage advice I would not be where I am today. So thank you for taking a chance on me after that first meeting and letting me into your lab to work, learn and grow.

Next, I would like to thank my committee members André Gobin, PhD. and Gerold Willing, PhD. for providing helpful suggestions when faced with constant thesis related struggles. Also thank you for letting me into your labs to do research. For without your alls equipment and tools this project would not have been possible.

I would also like to thank the bioengineering faculty and staff, especially Robert Keynton $\mathrm{PhD}$, for providing me with the tools and instruction necessary to complete a thesis. I also want to thank my class for the last five years and their help along the way.

I would also like to thank my parents Don and Debra Strotman for being there to always support me and provide meaningful advice. I would also like to thank my sister, Katie Strotman, for always telling me to relax.

Lastly, I would like to thank my boyfriend, Aaron Dewitt, for listening and always encouraging me in everything I do. Without you as a support system, I would not 
be the person I am today. Thank you for always being there and letting me lean on you no matter the circumstance. 


\begin{abstract}
Catheters represent an essential part of the management of critically and chronically ill patients. However, their use is often plagued by catheter related blood stream infections (CRBSIs), which are associated with increased morbidity, hospital stay and medical costs. Treatment for CRBSIs is often difficult due to the microorganism's development of resistance to the drug being used. Development of the resistance has been directly correlated with the formation of biofilms, caused when bacteria adhere to the surface of the catheters in community-like complexes. In order, to overcome antibiotic resistance, chitosan nanoparticles encapsulating a biofilm degrading enzyme, $\beta-\mathrm{N}$ Acetylglucosaminidase (NAGase) were fabricated through an ionic gelation method. Chitosan nanoparticle parameters; including, size, zeta potential, morphological characteristics, swelling properties, encapsulation efficiencies and release profiles were optimized for use as a biofilm degrading enzyme carrier. We hypothesize that sustained release of NAGase for biofilm degradation can reduce the formation of biofilms and increase the effectiveness of antibiotics to aid in reducing CRBSIs.
\end{abstract}




\section{TABLE OF CONTENTS}

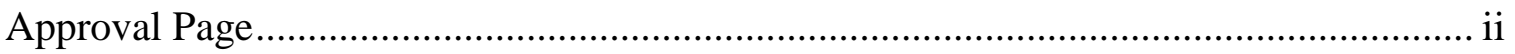

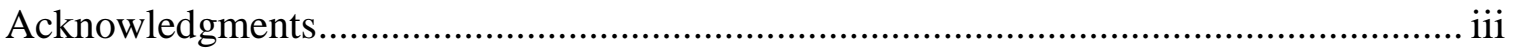

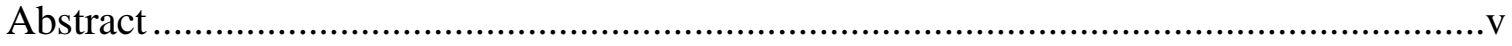

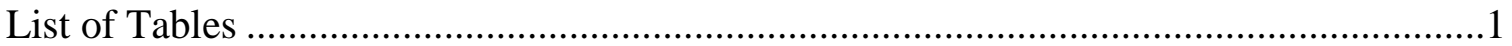

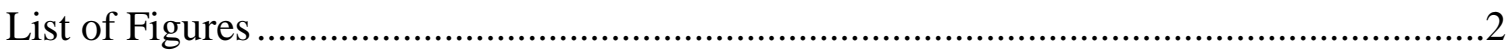

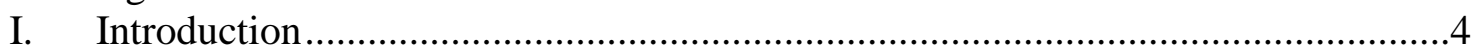

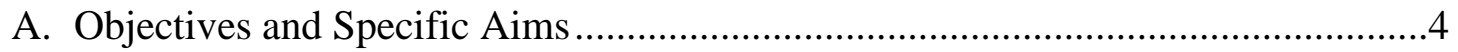

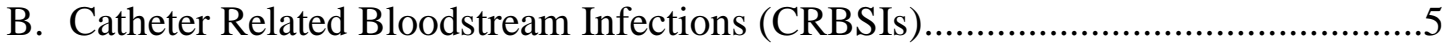

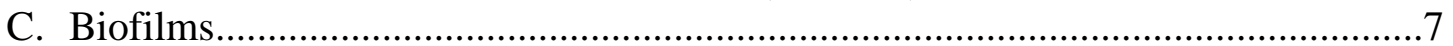

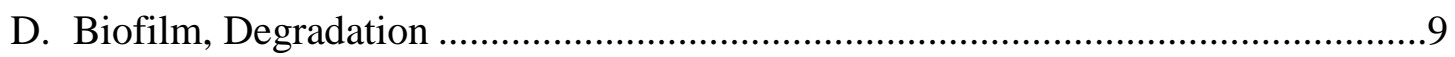

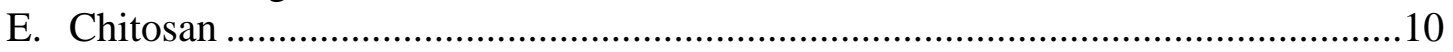

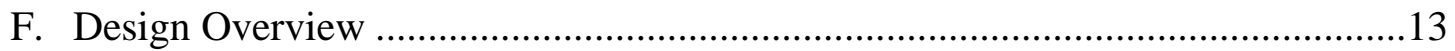

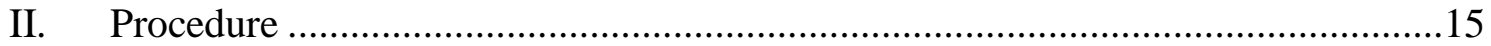

A. Fabrication of Chitosan Particles ................................................................... 16

1. Low Molecular Weight Chitosan Particles ..............................................18

2. High Molecular Weight Chitosan Particles ............................................19

3. Heppe Medical Chitosan Particles ...........................................................19

B. Size and Zeta Potential Measurement of Chitosan Particles ................................19

C. Determination of Chitosan Particle Seperation Method .......................................21

1. Low Molecular Weight Chitosan Particles ...........................................21

2. Heppe Medical Chitosan Particles ............................................................23

D. Determination of Crosslinker Methods for Chitosan Particle ..................................23

1. Trisodium Citrate Crosslinker................................................................24

2. GPTMS Crosslinker.......................................................................25

3. Genipin Crosslinker .....................................................................26

4. Heppe Medical Chitosan Genipin Crosslinker .......................................27

E. Morphological Characterization of Chitosan Particles .....................................29

1. Low Molecular Weight Chitosan Particles ............................................29

2. Heppe Medical Chitosan Particles ..........................................................29

F. Different pH TPP Solution Effect on Chitosan Particle Size ................................30

G. Swelling Effect on Chitosan Particles in Various $\mathrm{pH}$...........................................30

1. Low Molecular Weight Chitosan Particles ...............................................30

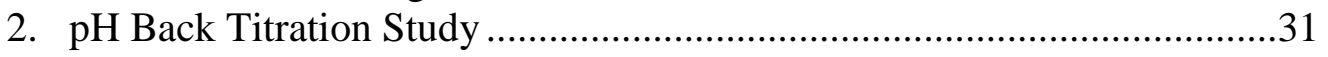

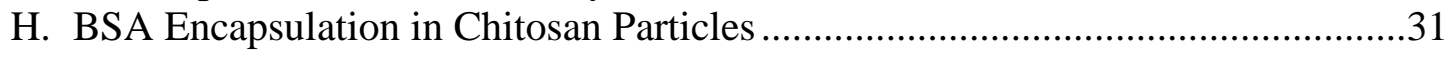

I. BSA Release from Chitosan Particles..............................................................33 
1. Low Molecular Weight Chitosan Particles ..............................................33

2. Heppe Medical Chitosan Particles ...........................................................35

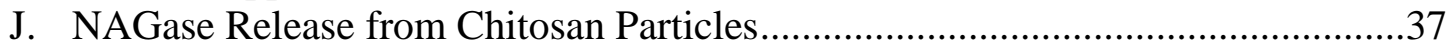

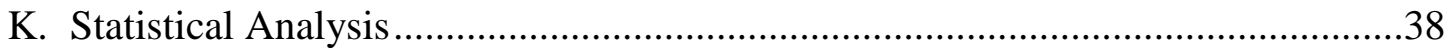

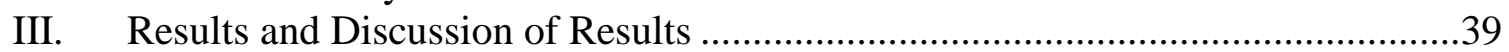

A. Fabrication Size Measurement of Chitosan Particles ......................................40

1. Size Measurements of Chitosan Particles ...............................................40

a. Low Molecular Weight Chitosan Particles ...................................40

b. High Molecular Weight Chitosan Particles .................................43

c. Heppe Medical Chitosan Particles .............................................44

d. Different pH TPP Solutions Effect on Chitosan Particle Size ......47

2. Zeta Potential Measurement of Chitosan Particles ..................................48

a.Low Molecular Weight Chitosan Particles ...................................48

b. Heppe Medical Chitosan Particles .............................................50

3. Determination Chitosan Particles Seperation Method ............................51

a.Low Molecular Weight Chitosan Particles ..................................52

b. Heppe Medical Chitosan Particles ..............................................54

4. Morphological Characterization of Chitosan Particles .............................56

5. $\mathrm{pH}$ Swelling Study Effect on Chitosan Particles ....................................59

a.Low Molecular Weight Chitosan Particles .................................59

b. pH Back Titrate Study ...............................................................61

B. Modulation of Chitosan Particles Via Crosslinking ..........................................62

1. Trisodium Citrate Crosslinker..........................................................62

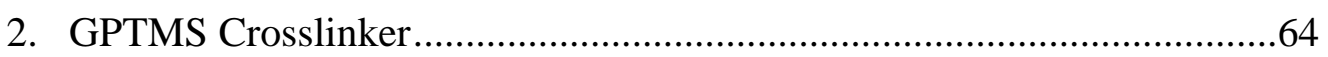

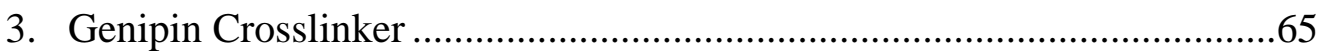

4. Heppe Medical Chitosan Particles Crosslinked With Genipin ..................68

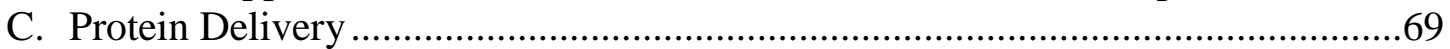

1. BSA Encapsulation in Chitosan Particles ............................................69

2. BSA Release From Chitosan Particles....................................................72

a.Low Molecular Weight Chitosan Particles .................................. 72

b. Heppe Medical Chitosan Particles .............................................77

3. NAGase Release From Chitosan Particles.................................................78

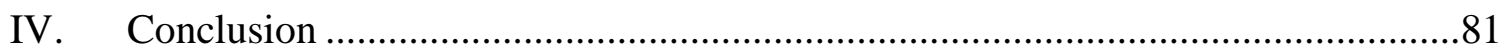

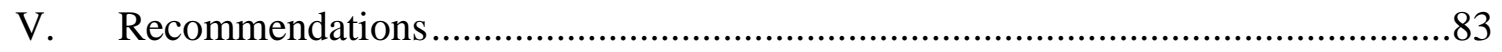

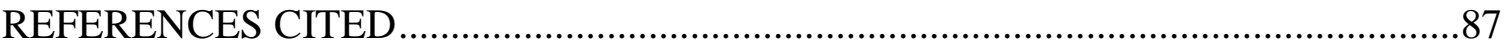

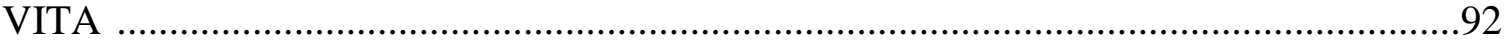




\section{LIST OF TABLES}

Table I: Chitosan Heppe Medical Chitosan Source Information ....................................17

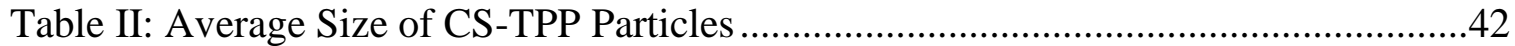

Table III: Comparison of Average Diameter of LMW Chitosan Particles .......................43

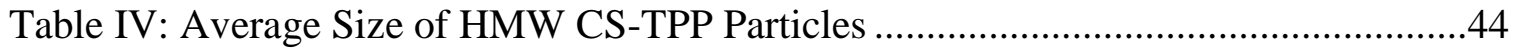

Table V: Average Size for $0.125 \% \mathrm{CS}$ and $0.1 \%$ TPP ...............................................46

Table VI: Zeta Potential (mV) of LMW CS-TPP Particles ...........................................48

Table VII: Average Zeta Potential $(\mathrm{mV})$ of Chitosan Particle Comparison ......................50

Table VIII: Zeta Potential (mV) Over Time ................................................................50

Table IX: Zeta Potential Heppe Medical Chitosan Particles ........................................51

Table X: Rotavap LMW CS Particles Average Diameter (nm) ...................................52

Table XI: Particle Size (nm) $0.5 \%$ CS-0.5 \% TPP for 30 Minutes at $10{ }^{\circ} \mathrm{C}$....................53

Table XII: Centrifugation Study with Genipin .........................................................54

Table XIII: Chitosan Particle Size Comparison Between SEM and DLS Measurements.56

Table XIV: pH Back Tritration Study .....................................................................62

Table XV: Swelling Response of CS Particles Crosslinked with Sodium Citrate ...........63

Table XVI: CS Particle Diameter (nm) Crosslinked with GPTMS ..............................65

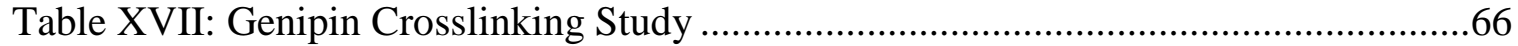

Table XVIII: Genipin Crosslinking Study (TPP Adjusted to $\mathrm{pH} 4.5$ ) ...........................67

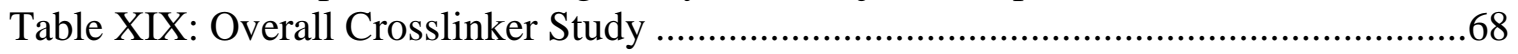




\section{LIST OF FIGURES}

Figure 1: $\beta$-N-Acetylglucosaminyl Linkage in Straphylococcus Epidermis ....................10

Figure 2: Chemical Structure of Chitosan .............................................................. 11

Figure 3: Chemical Structure of Chitin................................................................. 11

Figure 4: Ionic Gelation for Formulation of Chitosan Particles ....................................13

Figure 5: Ionic Gelation Method for Chitosan Particle Formation ..................................18

Figure 6: Chitosan Particle Separation by Centrifugation ...........................................23

Figure 7: Sodium Citrate Crosslinker Method for Chitosan Particles .............................25

Figure 8: GPTMS Crosslinker Method for Chitosan Particles ........................................26

Figure 9: Genipin Crosslinker Method for LMW Chitosan Particles...............................27

Figure 10: Genipin Crosslinker Method for Heppe Medical Chitosan Particles ...............28

Figure 11: BSA Before and After Chitosan Particle Encapsulation Method.....................36

Figure 12: Enlargened SEM Image of Heppe Medical Chitosan Particles........................40

Figure 13: Size of Chitosan Particles Based on Varying Concentration (w/v) of LMW CS and TPP

Figure 14: Size of Chitosan Particles Based on Varying Concentration (w/v) of HMW CS and TPP.

Figure 15: Size of Chitosan Particles Based on Varying Concentration (w/v) of Heppe

Medical CS and TPP

Figure 16: Size of Chitosan Particles Based on Varying Concentration (w/v) of Heppe Medical .125\% CS and TPP.

Figure 17: Average Size of Chitosan Particles Based on Varying pH of TPP Solution....47

Figure 18: Zeta Potential at Varying Concentration of CS and TPP .............................49

Figure 19: Centrifugation Study for Heppe Medical Chitosan .......................................55

Figure 20: SEM Image of Chitosan Particles (0.25 \% TPP-0.25 \% CS) .........................57

Figure 21: SEM Image of Chitosan Particles (0.5 \% TPP-0.5 \% CS) .............................57

Figure 22: SEM Image of Chitosan Particles (1 \% TPP-1 \% CS) ....................................58

Figure 23: SEM Image of Heppe Medical Chitosan Particles ..........................................59

Figure 24: Chitosan Particles Diameter During First 24 Hours......................................60

Figure 25: Chitosan Particles Diameter Over 1 Month ................................................61

Figure 26: Chemical Structure of Trisodium Citrate .................................................63

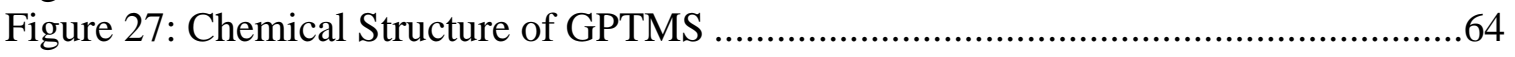

Figure 28: Chemical Structure of Genipin .................................................................65

Figure 29: Heppe Medical chitosan Particles Crosslinked with Genipin Study ...............69

Figure 30: BSA Encapsulation Efficiency Performed Spectroscopically.........................70 
Figure 31: BSA Encapsulation Efficiency Performed Via a BCA Assay

Figure 32: Confocal Image Encapsulating BSA After Chitosan Particle Formation .......72

Figure 33: Confocal Image Encapsulating BSA Before Chitosan Particle Formation .....72

Figure 34: Swelling Study of Chitosan Particles Encapsulating BSA...............................73

Figure 35: Swelling Study of Chitosan Particles Not Encapsulating BSA.........................74

Figure 36: Release Study of Chitosan Particles Measured Spectroscopically....................75

Figure 37: Release Study of Non-Stirred BSA Measured by Flourometer ........................76

Figure 38: Release Study of Stirred BSA Measured by Flourometer................................77

Figure 39: Heppe Medical Chitosan Particle Swelling Study …………….......................78

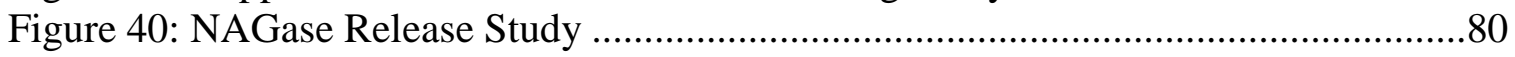




\section{INTRODUCTION}

\section{A. Objectives and Specific Aims}

Catheter related blood stream infections (CRBSIs) result in significant mortality and morbidity in critically ill patients. The Center for Disease Control and Prevention (CDC) has stated that there are approximately 250,000 cases per year (O'Grady M.D., et al., 2002). The precise economic impact is difficult to quantify and estimates have varied from $\$ 4,888$ to $\$ 56,167$ per patient in studies performed over the past decade (Goede M.D. \& Coopersmith M.D., 2009). The infections form when catheters become colonized by organisms from the skin, the environment or the bloodstream. They are often difficult to treat because the disease causing organisms embed themselves in a protective layer, called a biofilm, on the surface of the catheter that results in an increased resistance to antimicrobial agents (Dwyer, 2008). Therefore, in order to overcome the biofilm, a novel nanoparticle composed of chitosan encapsulating a biofilm degrading enzyme, $\beta-\mathrm{N}$ acetylglucosaminidase (NAGase) (Li \& Li, 1970) was developed. It is the hope that this therapy could help to reduce the number of CRBSIs per a year, the cost associated with the illness and provide long term protection against the risk of CRBSIs.

In order for the nanoparticles to become an effective therapy several specific aims must be met. First, the nanoparticles must be characterized and optimized for the 
application. This includes their size, zeta potential and morphological characterization. Second, the nanoparticles must exhibit $\mathrm{pH}$-dependent swelling properties; in order, to provide the needed burst release followed by sustained release. Finally, the actual release kinetics of the NAGase from the chitosan nanoparticles must be optimized.

\section{B. Catheter Related Blood Stream Infections (CRBSIs)}

Catheters represent an essential part of management of critically ill and chronically ill patients. However, their use is often complicated by serious infections, namely catheter related blood stream infections (CRBSIs), which are associated with increased morbidity, hospital stay and medical costs (Dwyer, 2008). Risk factors for CRBSIs include: reason for catheter use, length of catheter implantation, patient location (outpatient, inpatient floor, ICU), type of catheter, number of manipulations per a day, presence of needless connectors (increases risk), placement and whether or not best practices are followed (Goede M.D. \& Coopersmith M.D., 2009).

Current therapies, besides prevention practices; include, microbial impregnated catheters and dressings, silver impregnated catheters, aseptic catheter hubs and antimicrobial/anticoagulant flush solutions (Raad, Costerton, Sabharwal, Sacilowski, Anaissie, \& Bodey, 1993) (Goede M.D. \& Coopersmith M.D., 2009). The first therapy is the use of a sponge impregnated with chlorhexidine gluconate, which is applied at the catheter insertion site. It has been shown to reduce skin colonization and bacterial migration along the external surface of the catheter as compared with just skin disinfection techniques, but only for a few days. Also, there have been reports of local dermatitis. Another technique is the use of a silver impregnated collagen cuff attached to the catheter and left below the skin catheter insertion site. However, the clinical data as to 
its efficacy is conflicting and it was shown to make no difference after 20 days. The 20 day limit was thought to be due to either the collagen degrading or all the silver ions being released within 3-7 days. Lastly, this technique requires an experienced physician for insertion; in order, to limit the protrusion of cuff after insertion (Cicalini, Palmieri, \& Petrosillo, 2004). Other heavily researched therapies; include, catheters impregnated with antimicrobial agents. The first best studied impregnated catheter is one coated with chlorhexidine and silver sulfadiazine. It has been proven to decrease the risk for colonization by at least four fold as compared to non-coated catheters. The main limitation is the poor efficacy documented for long tern use (greater than 2 weeks). Logghe, accounted this to be due to the reduced activities of the antiseptics and lack of protection against external contamination (Logghe, Van Ossel, D'Hoore, Ezzedine, Wauters, \& Haxhe, 1997). As of now, no resistances to the antiseptic components have yet been documented; however, there have been reports of allergic reactions. The second well studied impregnated catheter is coated with minocycline-rifampin. This coated catheter has been shown to have a lower rate of infection than the chlorhexidine/silver sulfadiazine coated catheters. Also this type of coated catheter has been shown to retain antibiotic activity for greater than 2 weeks (Marciante, Veenstra, Lipsky, \& Saint, 2003). Currently, there have been no documented cases of antimicrobial resistances to this type of catheter in-vivo but there have been reports in in-vitro studies. It was suggested that this could be due to antibiotics being repeatedly exposed to the in-vitro bacteria without maintaining minimal therapeutic antibiotic use; therefore, the bacteria might be gaining mechanisms for antibiotic resistance (Cicalini, Palmieri, \& Petrosillo, 2004). Silver impregnated catheters are also widely used but a recently published study of a 
combination of other studies showed no evidence that it reduces catheter colonization (Goede M.D. \& Coopersmith M.D., 2009). Another possible therapy is a catheter hub containing iodinated alcohol solutions; however, this is only for internal catheter protection (Cicalini, Palmieri, \& Petrosillo, 2004). Finally, antimicrobial lock solutions have been explored. In this technique an antimicrobial solution, often containing an anticoagulant, is instilled in the lumen of the catheter and allowed to remain for 6-12 hours before it is removed. But due to the associated risk factors of the anticoagulants, it is not recommended for routine use ( $\operatorname{Raad} \&$ Hanna, 2002).

Several therapies have been shown to significantly decrease the risk of CRBSIs; however, most have only worked for the short term, less than 10 days (Karchmer, 2009). There are several reasons for this including but not limited to: all antimicrobial agents being released within the first few days, degradation or failure of the antimicrobial delivery system, repeated exposure to antibiotics resulting in antibiotic resistance and external contamination. Therefore, it is imperative to develop a safe and effective therapy against CRBSIs for long term catheter use in patients

\section{Biofilms}

The common bacteria involved in catheter related infections are S. epidermidis, $S$. aureus, Candida albicans, Pseudomonas aeruginosa, Klebsiella pneumoniae, and Enterococcus faecalis (Raad and Hanna 2001, Donlan 2001). These bacteria aggregate and anchor to catheter surfaces and form biofilms through substances they secrete. The biofilm then acts as a protective matrix with its growth correlating to antibiotic resistance, one of the main causes of failure for short and long term catheter failure (Dwyer, 2008). 
Biofilms are not necessary for bacteria to adhere to catheters, but they are essential for bacteria to colonize on the catheter surface. The biofilms are composed of water (up to $97 \%)$, polysaccharides $(1-2 \%)$ and proteins $(<1 \%)$, in which bacteria $(2-5 \%)$ encase themselves (Sutherland, 2001). They have channels and fluid filled voids that are approximately $1 \mu \mathrm{m}$ or less (Drury, Stewart, \& Characklis, 1993). These channels and voids are used to facilitate the flow of nutrients, enzymes, metabolites, waste products and other solutes (Sutherland, 2001). They can also lead to local accumulation of waste products due to differences in colony density, which can create microenvironments of differing pH (Stewart \& Costerton, 2001). This creates an architecture that is both heterogeneous and structurally complex. However, it is this architecture that will be exploited for the design of the chitosan nanoparticles. The first exploitation will be the chitosan nanoparticles small size, which will allow for easier entry into the small channels and voids. Secondly, the low pH microenvironments are also important; in order, for the $\mathrm{pH}$-dependent chitosan nanoparticles to provide a burst release of the encapsulated biofilm degrading enzyme, NAGase.

As mentioned previously, biofilm degradation is important to this application since both early and late catheter failure is due to its growth being correlated to antibiotic resistance. The antibiotic resistance is thought to be achieved through three main mechanisms. The first is the slow or incomplete penetration of the drug due to the ability of the biofilm to act as a protective layer (Stewart \& Costerton, 2001). The second is the possibility of a bacterial subpopulation differentiating into a unique phenotype that protects it from the effects of antibiotics (Sutherland, 2001). And third, is the local microenvironments mentioned previously, that have a low $\mathrm{pH}$ due to the bacteria's 
metabolism. These local $\mathrm{pH}$ gradient microenvironments can deactivate the antibiotic or causes the bacteria to enter a non-growing phase. These three mechanisms help the bacteria evade immune responses and antimicrobial therapy; however, when the biofilm is degraded the microorganisms rapidly become susceptible to the antibiotics, since all protection from the biofilm is lost (Stewart \& Costerton, 2001).

\section{Biofilm Degradation}

Biofilm degradation is essential; in order, to deliver therapeutic antibiotics that will not be inhibited. To degrade biofilms, chitosan nanoparticles will act as a drug delivery vehicle for the biofilm degrading enzyme $\beta$-N-acetylglucosaminidase (NAGase). NAGase is a common enzyme found in almost all tissues and organs. It has a molecular weight of $100 \mathrm{kDa}$ and its optimal $\mathrm{pH}$ range for enzymatic activity is 3.5 to $6(\mathrm{Li} \& \mathrm{Li}$, 1970). This enzymatic range is especially important, since the NAGase activity is only wanted and required at the lower $\mathrm{pH}$ ranges of the biofilm microenvironments. It is also important to note that NAGase can degrade the terminal ends of chitosan. But since it has minimal activity at higher $\mathrm{pHs}$ it should not affect the drug delivery vehicle until it reaches the low $\mathrm{pH}$ microenvironments of the biofilm. Once targeted the terminal degradation could be beneficial for a better burst release of the NAGase.

The main reason for using NAGase is its ability to hydrolyze $\beta-\mathrm{N}$ acetylglucosaminyl linkages found in amino-sugar containing compounds such as glycoproteins, glycolipids, blood group substances and bacterial cell walls ( $\mathrm{Li} \& \mathrm{Li}$, 1970). For example, Straphylococcus epidermidis as mentioned earlier represents one of the most frequent causes of CRBSIs. It has a very well characterized polysaccharide, within its biofilm, that consists of 80-85\% N-acetyl-D-glucosaminyl residues and 15-20 
$\%$ non-N-acetylated positively charged $\mathrm{n}$-glucosaminyl residues that are joined by the same $\beta$-N-acetylglucosaminyl linkage (Figure 1) that can be degraded by NAGase (Mack, et al., 1996)

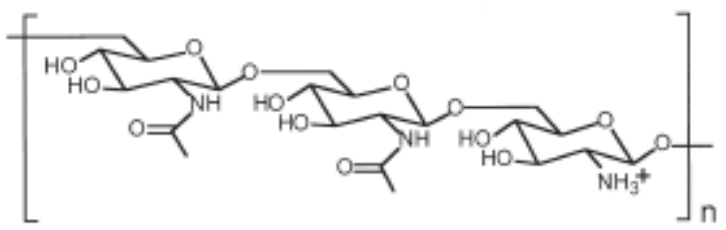

Figure 1 - $\beta$-N-acetylglucosaminyl Linkage in Straphylococcus epidermidis

The ability of NAGase to degrade biofilms has also been proven in other CRBSIs causing bacteria, such as Candida albicans (Al-fattani \& Douglas, 2006). In conclusion, the ability to degrade polysaccharides, one of the main components of biofilms, is essential in its overall degradation.

\section{E. Chitosan}

Chitosan (Figure 2) is made of linear $\beta(1 \rightarrow 4)$-linked glucosamine and $\mathrm{N}$-acetyl glucosamine units. It is obtained from deacetylation of chitin (Figure 3), a naturally occurring polysaccharide found abundantly in murine crustaceans, such as crabs and shrimp (Prabaharan \& Mano, 2005). Due to its abundance, low production cost, and intrinsic properties chitosan has become a choice material for medical and pharmaceutical applications; such as, carriers for drugs, protein carriers and gels for the entrapment of cells or antigens (Ko, Park, Hwang, Park, \& Lee, 2002). However, this introduction will only focus on chitosan nanoparticles as protein carriers. 


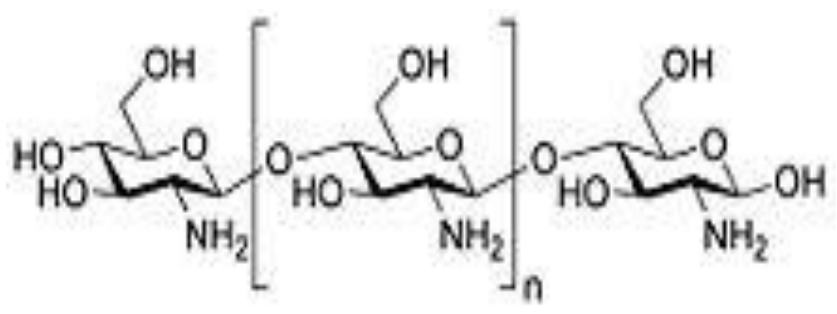

Figure 2: Chemical Structure of Chitosan

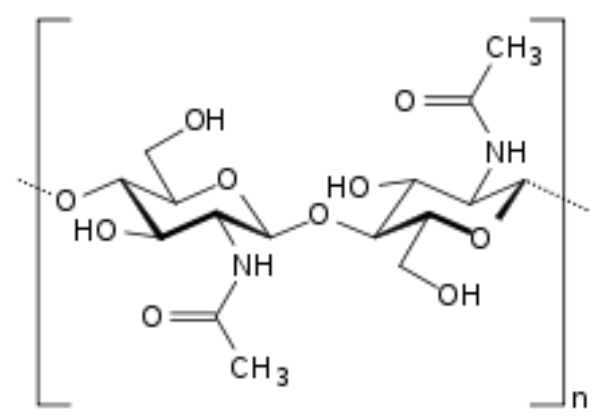

Figure 3 - Chemical Structure of Chitin

Chitosan has been receiving increased attention due to its intrinsic properties. The first important intrinsic property is chitosan's proven biocompatibility, which allows for topical application, injection or implantation Secondly, it is metabolized by a human lysosome, which makes it biodegradable (Berger, Reist, Mayer, Felt, Peppas, \& Gurny, 2004). Thirdly and perhaps most importantly for this application is that chitosan exhibits $\mathrm{pH}$ dependent swelling properties, which is essential to control the NAGase release (Gan, Wang, Cochrane, \& McCarron, 2005). Chitosan is also associated with a positive charge at physiological $\mathrm{pH}$, making it mucoadhesive. This helps increase retention at the site of application, which again is important for this application; in order, to maintain controlled drug release (Xu \& Du, 2003). Finally, chitosan has been proven to promote wound healing and has bateriostatic effects primarily through the inhibition of the transcription of DNA (Liu, Guan, Yang, Li, \& Yao, 2000). This bateriostatic effect could act as an 
additional inhibitor of biofilms making chitosan an even more attractive drug delivery vehicle.

Currently, there are several procedures to create chitosan particles. These techniques can be broken down into covalently or ionically cross-linked particles. The earliest work in chitosan nanostructures predominantly involved chemical cross-linking reactions to the polymer chain. Watzke and Dieschbourg (1994) formed chitosan-silica nanocomposites by reacting tetramethoxysilane (TMOS) to hydroxyl groups on the chitosan monomer. Later, Ohya (1994) and colleagues were the first to present data involving chitosan nanospheres for drug delivery applications using a water-in-oil emulsion method followed by cross-linking of the amino groups with glutaraldehyde. Although, these pioneering studies demonstrated feasibility of synthesizing stable, reproducible nanosized chitosan particles it was later discovered that the cross-linking agent, glutaraldehyde, had negative effects on cell viability and the integrity of macromolecular drugs. Therefore, general interest shifted to less harsh procedures for the synthesis of chitosan particles.

The shift was made to ionically cross-linked particles that exploited the cationic nature of chitosan and its ability to gel on contact with specific polyanions. Bodmeier (1989), first reported ionotropic gelation of chitosan with tripolyphosphate (TPP) for drug encapsulation to create microspheres. Calvo (1997) and colleagues later refined the method for nanoparticle (200-1000 nm) fabrication by the addition of an alkaline phase (pH 7-9) containing TPP with into an acidic phase (pH 5-6) containing chitosan (Figure 4). 


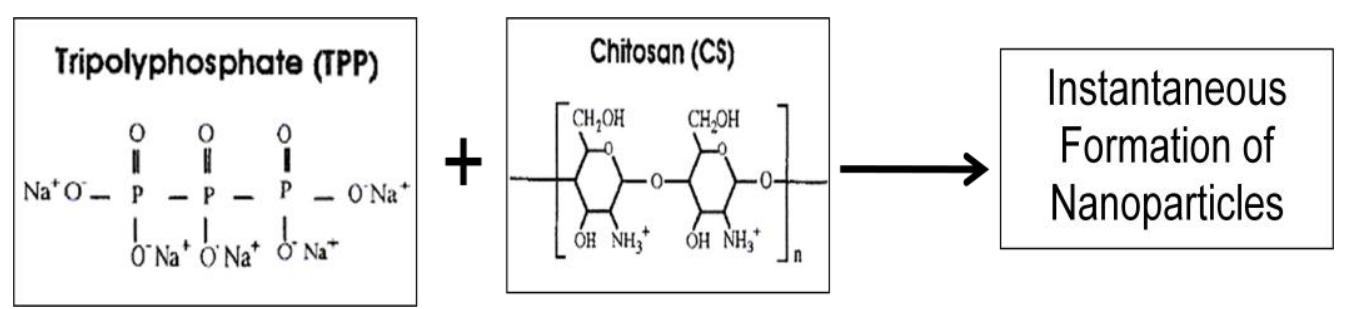

Figure 4 - Ionic Gelation for Formation of Chitosan Particles

For this method the nanoparticles form instantaneously upon mixing of the two solutions through inter and intramolecular linkages created between TPP phosphates and chitosan amino groups. This method presents several attractive features for a drug delivery vehicle because the process is simple and mild, meaning the fabrication process is easier and should not cause any inactivity or structural changes to the enzyme NAGase. Also the nanoparticle solution created is very homogenous and contains nanoparticles whose size and charge can easily be adjusted via concentration of TPP and chitosan; as well as, chitosan molecular weight and chitosan deacetylation degree (Calvo, Remunan, J.L.V., \& Alonso, 1997). Lastly, in addition to Calvo, there are several other published studies confirming the large capacity load for proteins in chitosan-TPP particles reference.

\section{F. Design Overview}

Due to the attractive features of the ionotropic gelation method for chitosan nanoparticles, it was used in the following studies as a possible protein carrier for NAGase. It is important to note that formation of nanoparticles via this method depends upon the purity of the chitosan, the acid needed for dissolution of the chitosan, and the molecular weight of the chitosan. Consequently, these conditions are different for every individual chitosan type; therefore, nanoparticles for this application will need to be optimized due to a difference in chitosan source from other published studies. It is also 
imperative that these particles be optimized for a size less than $1 \mu \mathrm{m}$ (Drury, Stewart, \& Characklis, 1993); in order, for the nanoparticles to diffuse easily into the biofilm. These nanoparticles must also exhibit swelling and degradation in a pH 5 environment, so that the enzyme NAGase will be released and active. Finally, burst release followed by sustained release of the NAGase must be shown for a continued therapeutic effect. Therefore, the following studies are focused on optimizing size, zeta-potential, morphological characteristics, swelling properties, encapsulation efficiencies and release profiles of chitosan-TPP nanoparticles for use as biofilm degrading enzyme carrier. 


\section{PROCEDURE}

Chitosan particles encapsulating NAGase were fabricated to degrade biofilms; in order, to overcome antibiotic resistant barriers in CRBSIs. The following sections represent procedures to fabricate the chitosan particles encapsulating NAGase; as well as, procedures to characterize, optimize and prove their efficacy for this particular application. It will be important to prove that the chitosan particles are nano-sized in to fit within the fluid filled microchannels of the biofilm; in order, to deliver the NAGase to an effective site. This was achieved through digital light microscopy and scanning electron microscopy. The zeta potential was also found to confirm the mucoadhesive properties of the chitosan and to confirm the chemistry of ionic gelation. The best separation procedure of chitosan particles was also determined so that they could be used in additional studies. Within the separation procedures the poor mechanical properties of chitosan particles was confirmed; therefore, additional studies with crosslinkers to improve mechanical strength was completed. It was also important to show is that the chitosan particles exhibited $\mathrm{pH}$ dependent swelling properties so that the NAGase can be released and active in the low pH biofilm microenvironments. Finally, the encapsulation and release of NAGase from the chitosan particles must be shown to prove the validity of the concept. It is also 
important to note that for some encapsulation and release studies BSA was used as a model protein for cost effectiveness reasons.

\section{A. Fabrication of Chitosan Particles}

The chitosan particles were obtained through ionic gelation of chitosan (CS) with tripolyphosphate (TPP). Preliminary experiments were conducted to discover the optimal production zone needed for CS particle formation, which should be less than $1 \mu \mathrm{m}$, with 200-400 $\mathrm{nm}$ being the desired CS particle size. The $1 \mu \mathrm{m}$ limit is based on the max size of the fluid filled channels in biofilms (Drury, Stewart, \& Characklis, 1993); therefore, if CS particles are larger they cannot diffuse into the biofilm for a more targeted NAGase release. The nano-sized CS particles were found by varying the molecular weight and deacetylation of chitosan; as well as, the concentration of the chitosan and TPP solution. The molecular weight is an important optimization parameter because higher molecular weights (>400 kDa) often cause the CS solution to be too viscous; therefore, causing CS to clump rather than form CS particles. However, if the chitosan molecular weight is too low $(<100 \mathrm{kDa})$ than the encapsulation efficiencies of the drug of interest is too low. These properties were expected and are related to linear chain length of the chitosan. Deacetylation, the removal of an acetyl group, is important so that the amino group is exposed. It is critical this group be exposed since ionic gelation depends on the positive charge of the amino group and the negative groups of the TPP. Therefore, the higher deacetylation percentages allow for more crosslinking to occur in the ionic gelation procedure.

These experiments were completed with both low and high molecular weight chitosan. The low (LMW) and high (HMW) molecular weight chitosan was purchased 
from Sigma Aldrich (St. Louis, MI), which was $91.8 \%$ and $75.6 \%$ deactylated with a viscosity of $32 \mathrm{cps}$ and $1406 \mathrm{cps}$. In later experiments, chitosan produced within a narrower deacetylation percentage and molecular weight was obtained from Heppe Medical Chitosan (Germany). The chitosan was approximately $90 \%$ deactylated with three different molecular weights of 150,250 and $350 \mathrm{kDa}$ (Table I). This switch of chitosan manufacturers was critical to produce CS particles that were of constant size and low polydispersity; in order, to increase reproducibility.

\begin{tabular}{|c|c|c|c|}
\hline \multicolumn{4}{|c|}{ TABLE I } \\
\hline CHITOSAN HEPPE MEDICAL CHITOSAN SOURCE INFORMATION \\
\hline & 1 & 2 & 3 \\
\hline Molecular Weight & 150 & 250 & 350 \\
\hline Viscosity (cps) & 36 & 250 & 680 \\
\hline Deacetylation \% & 92.1 & 88.1 & 88.7 \\
\hline
\end{tabular}

It is also important to note that the exact molecular weight of chitosan was not known for the Sigma Aldrich chitosan, as only the viscosities were reported. The method for CS particle fabrication, no matter the type of chitosan used, is shown below in Figure 5. 
\% CS Solution: Dissolve in D.I. water with an acetic acid concentration 1.75 times that of the CS concentration

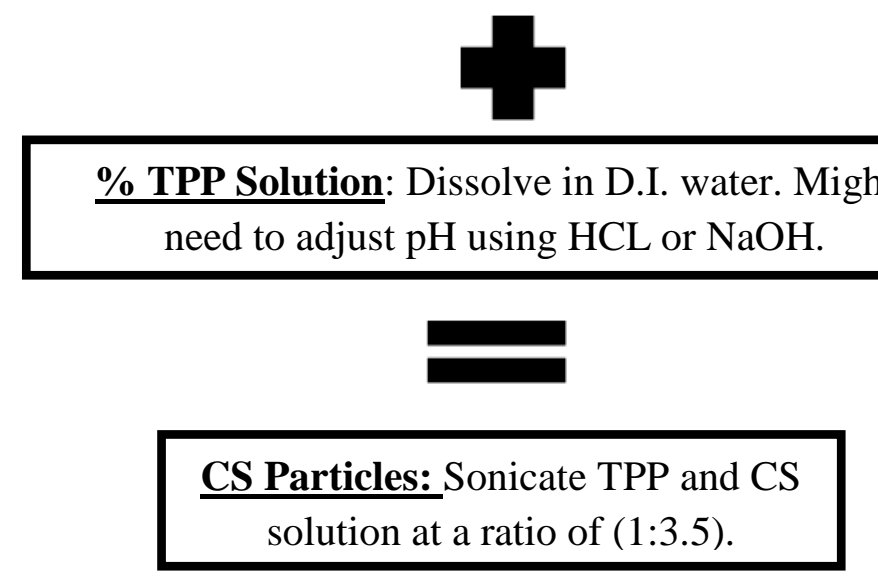

Figure 5 - Ionic Gelation Method for Chitosan Particle Formation

\section{Low Molecular Weight Chitosan Particles}

For LMW chitosan, CS was dissolved in 99.7+\% acetic acid (Sigma Aldrich St. Louis, MI) aqueous solution at various CS concentrations: $0.125 \%, 0.25 \%, 0.5 \%, 1 \%$, and $2 \%(\mathrm{w} / \mathrm{v})$. The concentration of acetic acid was 1.75 times higher than that of CS in all cases, which resulted in a solution $\mathrm{pH}$ of approximately 3.5. Next, TPP (Sigma Aldrich, St. Louis, MI) was dissolved in deionized (D.I.) water at the same concentrations as $\operatorname{CS}(0.125 \%, 0.25 \%, 0.5 \%, 1 \%$, and $2 \%(w / v))$. Lastly, the TPP solution was slowly pipetted into the CS solution for a 1:3.5 (TPP:CS) ratio while being sonicated (Microson XL 2000, Misonix, Farmingdale, NY) at 5 Watts (W) at room temperature $\left(25^{\circ} \mathrm{C}\right)$. Some studies which will be noted later adjusted the $\mathrm{pH}$ of the TPP solution to 4.5 using various concentrations of hydrochloric acid (Fisher Scientific, Fair Lawn, NJ) or sodium hydroxide (Fisher Scientific, Fair Lawn, NJ). 


\section{High Molecular Weight Chitosan Particles}

For HMW chitosan, only 0.25 and $0.5 \%(\mathrm{w} / \mathrm{v})$ concentrations of CS solutions were used. The concentration of acetic acid remained 1.75 times that of the CS \% concentration. The concentrations of TPP solutions were $1.5 \%, 2 \%$ and $2.5 \%(\mathrm{w} / \mathrm{v})$ and the $\mathrm{pH}$ of the solutions were adjusted to $\mathrm{pH} 4.5$ every time, using sodium hydroxide and hydrochloric acid. The changes in percentages of CS solution were needed so that the solution was not too viscous for particle formation. The change in TPP solution was also made based on the assumption there would be more amino groups that needed to be crosslinked for CS particle formation. Lastly, the TPP solution was slowly pippeted to the CS solution at a 1:3.5 (TPP:CS) ratio while being sonicated at $5 \mathrm{~W}$ at room temperature $\left(25^{\circ} \mathrm{C}\right)$.

\section{Heppe Medical Chitosan Particles}

To find the optimal production zone for Heppe Medical Chitosan particles, three factors were varied; including: molecular weight of chitosan (150, 250 \& $350 \mathrm{kDa})$, chitosan $\%$ concentrations $(0.125,0.25,0.5$ and $1 \%)$ and TPP $\%$ concentrations $(1,2.5$, 5, 10 and $15 \mathrm{mg} / \mathrm{mL}$ ). The CS particles were then created using the same procedure as described for HMW chitosan. It is important to note that the acetic acid concentration, and ratio of CS added to TPP remained the same, 1:3.5 (TPP:CS).

\section{B. Size and Zeta Potential Measurement of Chitosan Particles}

Size and zeta-potential measurements of the CS particles were performed using a Zetasizer Nano ZS90 (Malvern Instruments, England). Size and zeta potential measurements were taken for the different types of chitosan used to fabricate CS particles 
as described in the procedure above. Size measurements were also used several times as a critical analysis tool for other studies as described in other sections.

To determine size (diameter), dynamic light scattering capabilities of the ZetaSizer Nano ZS90 were exploited. To use, $1 \mathrm{~mL}$ of CS particles was pipetted into a low volume disposable sizing cuvette “ZEN0112” (Malvern Instruments, England) and placed at a $90^{\circ}$ angle to the detector. The measurement was taken with a preprogrammed standard operating protocol (SOP) file. To program the file, start a manual measurement and set the following parameters.

- Material: Polystyrene latex

○ R.I.: 1.590

○ Absorption: 0.01

- Dispersant: Water (Note: Acetic acid concentration so low does not affect

$$
\begin{aligned}
& \text { measurement value) } \\
& \text { ○ Temperature: } 25^{\circ} \mathrm{C} \\
& \circ \quad \text { Viscosity: } .8872 \mathrm{cps} \\
& \circ \quad \text { R.I. } 1.330
\end{aligned}
$$

To find zeta potential, $1 \mathrm{~mL}$ of CS particles is pipetted into the clear disposable zeta cell cuvette (Malvern Instruments, England). The measurement was taken with a different preprogrammed standard operating protocol (SOP) file. To program the file, start a manual measurement and set the following parameters.

- Material: Chitosan

○ R.I.: 1.528

○ Absorption: 0.00 
- Dispersant: Water (Note: Acetic acid concentration so low does not affect measurement value)

○ Temperature: $25^{\circ} \mathrm{C}$

○ Viscosity: .8872 cps

○ R.I. 1.330

Cuvette can be reused up to ten times. Before each reuse rinse with D.I. Water, Ethanol (Pharmco, Brookfield, CT), D.I. Water and then dry with an air hose.

\section{Determination of Chitosan Particle Separation Method}

Several methods were investigated to determine the best method for CS particle separation. This was essential; in order, for CS particles to be used in additional studies were the CS particles needed to be transferred to different buffers i.e. swelling studies. It is also important to note that the method of separation was difficult due to the poor mechanical properties of the CS particles and their fabrication efficiencies. Methods investigated; included rotary evaporation and high speed centrifugation.

\section{Low Molecular Weight Chitosan Particles}

For these methods, CS particles composed of $0.5 \%$ TPP and $0.5 \%$ LMW CS solutions were fabricated and their size measured as described in sections A and B $(\S \mathrm{A} \&$ $\S \mathrm{B})$. For rotary evaporation, the sample was dialyzed against D.I. water (Spectra/Por Dialysis Membrane MWCO: 6-8,000 kDa; Rancho Dominguez, CA) for 24 hours. The CS particle solution was then transferred to a rotavap (Buchi, New Castle, DE) until the water solution was completely evaporated, leaving only the CS particles. These CS particles were then resuspended in $1 \mathrm{~mL}$ D.I. water and their size measured for comparison $(\S \mathrm{B})$. The second method involved high speed centrifugation (Sorvall RC6, 
Kendro, Asheville, NC). The CS particle sizes were first measured $(\S \mathrm{B})$, then $1 \mathrm{~mL}$ of the CS particle solution was transferred to a $1.5 \mathrm{~mL}$ microcentrifuge tube and centrifuged at different time intervals (15 \& 30 minutes) and speeds (1950, 8230, 12860, 18510, 28930, 39830 \& $51430 \mathrm{x}$ g's). The temperature was always kept at $10^{\circ} \mathrm{C}$. Some CS particle samples were prepared with the addition of $20 \mu \mathrm{L}$ of glycerin (Fisher Scientific, Fair Lawn, NJ) before CS particles were added. After centrifugation, the supernatant was removed and measured $(\S \mathrm{B})$. The CS particles were then resuspended in $1 \mathrm{~mL}$ D.I. water. Finally, the CS particle sizes were again measured $(\S B)$. The experiment was repeated when $0.5 \mathrm{mM}$ genipin was added as a crosslinker and the TPP solution $\mathrm{pH}$ was adjusted to 4.5. However, within the second study no glycerin was added and the time intervals were changed (30 and 60 minutes), while the speeds were also changed (10,000, 20,000, $40,000 \mathrm{x}$ g's). It is important to note for all subsequent LMW CS studies the CS particles will be centrifuged at $40,000 \mathrm{x}$ g's for 30 minutes at $10^{\circ} \mathrm{C}$ unless otherwise noted. Figure 6 shows the procedural steps for this and all subsequent centrifugation studies. 


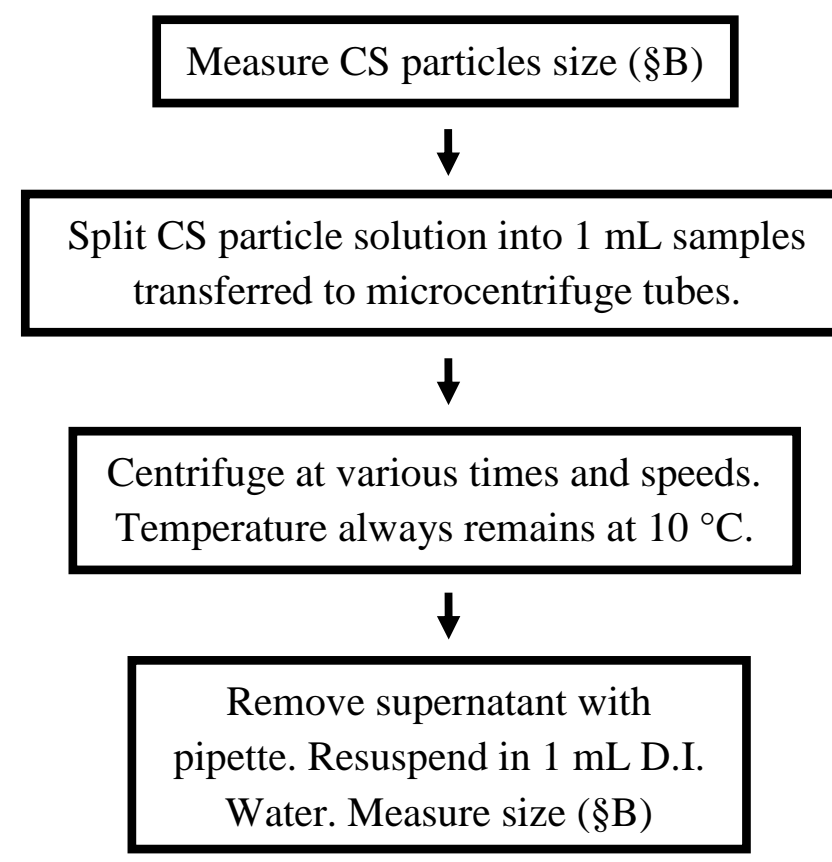

Figure 6 - Chitosan Particle Separation by Centrifugation

\section{Heppe Medical Chitosan Particles}

For the Heppe Medical Chitosan, $1 \mathrm{~mL}$ CS particles composed of $0.1 \%$ TPP at $\mathrm{pH} 4.5$ and $0.125 \% \mathrm{CS}_{250}$ were fabricated as described in section $\mathrm{A}(\S \mathrm{A})$ and then transferred to $1.5 \mathrm{mM}$ microcentrifuge tubes. The CS particle sizes were measured before centrifugation $(\S \mathrm{B})$. Next, the CS particles were centrifuged at different time intervals (5, 30 and 60 minutes) and speeds (12860, 18510, 28930, $39830 \mathrm{x}$ g's). The temperature remained at $10^{\circ} \mathrm{C}$. After centrifugation, the supernatant was removed and measured $(\S \mathrm{B})$. The CS particles were then resuspended in $1 \mathrm{~mL}$ D.I. water. Finally, the CS particle sizes were again measured $(\S \mathrm{B})$. For all subsequent studies involving Heppe Medical CS particles centrifugation will be performed at $39,830 \mathrm{x}$ g's for 60 minutes at $10{ }^{\circ} \mathrm{C}$.

\section{Determination of Crosslinker Methods for Chitosan Particles}

In addition to TPP, three additional non-toxic crosslinkers were explored to increase mechanical strength of the CS particles; including, sodium citrate (Fisher 
Scientific, Fair Lawn, NJ), genipin (Wako, Richmond, VA) and 3-

glycidoxypropyltrimethoxysilane "GPTMS" (Sigma Aldrich, St. Louis, MI). To analyze the crosslinkers effects, a centrifugation size study and swelling study were performed. Meaning, the size before centrifugation was measured $(\S \mathrm{B})$. The CS particles were centrifuged as described in section $\mathrm{C}(\S \mathrm{C})$. Next, after centrifugation, the supernatant was removed and the CS particles were resuspended in $1 \mathrm{~mL}$ D.I. water, phosphate buffered saline "PBS" (pH 7) (Fisher Scientific, Fair Lawn, NJ), sodium citrate buffer (pH 5) and PBS (pH 2). The sizes at hour 0 and 24 were then measured $(\S B)$. The size data collected at hour 0 of CS particles resuspended in D.I. water was also used for the after centrifugation data. It is important to note that all studies were carried out using a $0.5 \%$ TPP with no $\mathrm{pH}$ adjustments and a $0.5 \%$ LMW chitosan solution.

\section{Trisodium Citrate Crosslinker}

For sodium citrate, the CS particles were fabricated in a procedure similar to that described in section A (§A). A solution containing $0.5 \%(\mathrm{w} / \mathrm{v})$ sodium citrate and $0.5 \%$ TPP was mixed. The $0.5 \% \mathrm{TPP} /$ sodium citrate solution was slowly pipette, during sonication, into the $0.5 \%$ LMW CS solution at the same 1:3.5 (TPP/SodiumCitrate:CS) ratio. The process steps are described in Figure 7. 
\% LMW CS Solution: Dissolve in D.I. water with an acetic acid concentration 1.75 times that of the

CS concentration

\% Sodium Citrate/TPP Solution: Dissolve sodium citrate and TPP at a $0.5 \%(\mathrm{w} / \mathrm{v})$ concentration in D.I. water. Mix

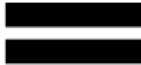

CS Particles: Sonicate Sodium Citrate/TPP and CS solution at a ratio of (1:3.5).

Figure 7 - Sodium Citrate Crosslinker Method for Chitosan Particles

\section{GPTMS Crosslinker}

The GPTMS crosslinker is added in two different methods. For the first method, a solution containing $0.5 \%(\mathrm{v} / \mathrm{v})$ GPTMS and $0.5 \%$ TPP was mixed. The $0.5 \%$

TPP/GPTMS solution was slowly pipetted, during sonication, into the $0.5 \%$ LMW CS at the same 1:3.5 (TPP/GPTMS:CS) ratio during sonication. The resulting solution was continuously mixed on a magnetic stir plate (Barnstead International, Dubuque, IW) for 24 hours. For the second method, GPTMS was added to D.I. water for a final concentration $0.5 \%(\mathrm{v} / \mathrm{v})$. Then equal volumes were mixed with previously fabricated CS particles composed of $0.5 \%$ TPP and $0.5 \%$ LMW CS (1:3.5) solutions on a magnetic stir plate for 24 hours. See Figure 8 for process steps. 


\section{5 \% LMW CS Solution: Dissolve in D.I. water}

with an acetic acid concentration 1.75 times that of the CS concentration

Method 1: Dissolve GPTMS $(\mathrm{v} / \mathrm{v})$ and TPP (w/v) at a $0.5 \%$ concentration in D.I. water. Mix

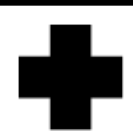

Method 2: Dissolve TPP at a $0.5 \%(\mathrm{w} / \mathrm{v})$ concentration in D.I. water. Mix

CS Particles: Sonicate solutions at a 1:3.5 (TPP/GPTMS:CS) ratio

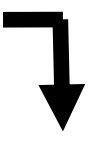

Method 2: Dissolve GPTMS in D.I. Water at a $0.5 \%(\mathrm{v} / \mathrm{v})$ concentration. Add to CS particle solution at a 1:3.5 (GPMTS:TPP/CS) ratio and mix for 24 hours.

Figure 8 - GPTMS Crosslinker Method for Chitosan Particles

\section{Genipin Crosslinker}

Genipin is insoluble in water; therefore, $1.13 \mathrm{mg}$ of genipin was added to $1 \mathrm{~mL}$ of ethanol. After dissolution, the solution was transferred to $9 \mathrm{~mL}$ D.I. water for a final genipin concentration of $0.5 \mathrm{mM}$. For the first method no TPP was added. Instead, the 0.5 mM genipin solution was added to the $0.5 \% \mathrm{CS}$ solution at a ratio of 1:3.5 (genipin:CS) and continuously stirred for 24 hours. The second method added the $0.5 \mathrm{mM}$ genipin solution to the $0.5 \%$ TPP solution at a ratio of 1:1 (genipin:TPP). The genipin/TPP solution is then mixed with the $0.5 \%$ LMW CS solution at a ratio 1:3.5 (TPP/genipin:CS) and continuously stirred for 24 hours. The third method added the 0.5 mM genipin solution after $0.5 \%$ TPP and 0.5 \% LMW CS (1:3.5) particle formation at 
the same ratio of TPP added. The solution was continuously stirred for 24 hours. It is important to note that this study was repeated twice; however, the $\mathrm{pH}$ of the $0.5 \%$ TPP solution was lowered to $\mathrm{pH} 4.5$ using sodium hydroxide. See Figure 9 for process steps.

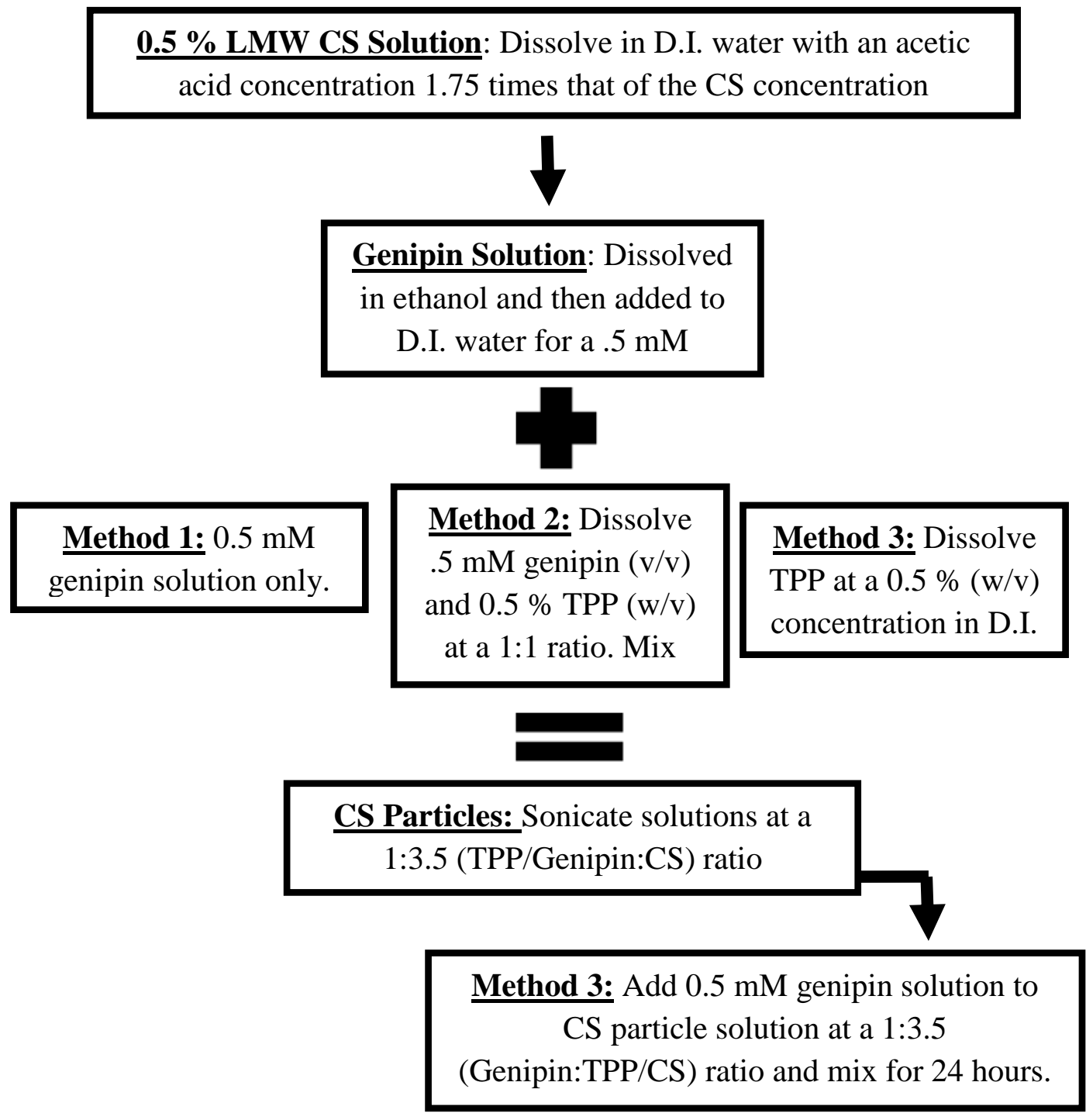

Figure 9 - Genipin Crosslinker Method for LMW Chitosan Particles

\section{Heppe Medical Chitosan Genipin Crosslinker}

For the Heppe Medical Chitosan, the size difference crosslinked with a $1 \mathrm{mM}$ genipin solution was also determined. It is important to note that a concentrated genipin 
solution was created for direct addition to the CS particle solution; instead of first being diluted in water. The genipin solution was created by dissolving $1 \mathrm{~g}$ genipin in $5 \mathrm{~mL}$ of ethanol. From there the genipin solution was added to $7 \mathrm{~mL}$ of a CS particle solution composed of $0.1 \% \mathrm{TPP} \mathrm{pH} 4.5$ and $0.125 \% \mathrm{CS}(\S \mathrm{A})$, for a final genipin concentration of $1 \mathrm{mM}$. The concentration of genipin was increased from $.5 \mathrm{mM}$ in previous studies to $1 \mathrm{mM}$ to determine if it caused a more dramatic effect on size of the CS particles both before and after centrifugation $(\S \mathrm{C})$. The resulting solution was continuously stirred for 24 hours on a rotating shaker. See Figure 10 for process steps.

0.125 \% Heppe Medical CS Solution: Dissolve in

D.I. water with an acetic acid concentration 1.75 times that of the CS concentration $0.5 \%(\mathrm{w} / \mathrm{v})$ concentration in D.I. water. Mix

CS Particles: Sonicate solutions at a 1:3.5 (TPP/:CS) ratio

Genipin dissolved in ethanol at a $20 \%(\mathrm{w} / \mathrm{v})$ concentration
Add genipin solution to CS particle solution for a $1 \mathrm{mM}$ concentration

Figure 10 - Genipin Crosslinker Method for Heppe Medical Chitosan Particles 


\section{E. Morphological Characterization of Chitosan Particles}

\section{Low Molecular Weight Chitosan Particles}

Morphological features were examined via scanning electron microscopy (SEM: Evo 40, Zeiss, Germany). Different CS particle solution composed of either $0.25,0.5$ or $1 \%$ TPP and $0.25,0.5$ or $1 \%$ LMW CS were used for this morphological characterization study. For preparations of samples, a droplet of homogenous CS particle solution was prepared on a copper grid having a mesh of 300 (Election Microscopy Sciences, Hatfield, PA). The CS particles were then allowed to air dry at room temperature for 24 hours. All samples were then coated with gold palladium, at a thickness of approximately $100 \AA$ for SEM (FEI Nova NanoSEM, Hillsboro, OR) observation. It is important to note that all images were taken at a low voltage of $2 \mathrm{kV}$, due to the charging effects causing the samples to bleach out. The CS particle size distribution and morphology were then analyzed using Image $\mathbf{J}(\mathrm{NIH})$.

\section{Heppe Medical Chitosan Particles}

SEM pictures of the Heppe Medical Chitosan particles, composed of $0.1 \%$ TPP $\mathrm{pH} 4.5$ and $0.125 \% \mathrm{CS}_{250}$, were taken. A droplet of homogenous CS particle solution, was prepared on a copper grid. The CS particles then adhered to the surface for 5 minutes before some of the CS particle suspension liquid was removed via fluid absorption with a kimwipe. The CS particles were then allowed to air dry for 24 hours. All preparations were then coated with gold palladium at a thickness of approximately $100 \AA$ for SEM observation. Pictures were taken at $6 \mathrm{kV}$, using a different SEM to determine if imaging difficulties were due to instrumentation. 


\section{F. Different pH TPP Solutions Effect on Chitosan Particle Size}

The following study was created to determine the size effects of a TPP solution with a varying $\mathrm{pH}$. This was done based on previous literature that states when the $\mathrm{pH}$ of the TPP solution is lowered, the diameter of the CS particles decreases (Gan, Wang, Cochrane, \& McCarron, 2005). A $0.5 \%$ TPP solution was created and its $\mathrm{pH}$ adjusted to 4, 4.5 or 5 using sodium hydroxide or hydrochloric acid. CS particles where than fabricated with a $0.5 \%$ LMW CS as described in section A (§A). The $0.5 \%$ TPP solution was sonicated with the $0.5 \%$ LMW CS solution at a 1:3.5 (TPP:CS) ratio. The diameter of the subsequent CS particles was measured $(\S B)$. For the Heppe Medical Chitosan, the size difference for TPP solutions at $\mathrm{pH} 4.5$ and 9 was also determined.

\section{G. Swelling Effect on Chitosan Particles in Various pH}

\section{Low Molecular Weight Chitosan Particles}

CS particles composed of $0.5 \%$ TPP and $0.5 \%$ LMW CS were created in a large batch by maintaining the same ratios of CS to TPP as described in the CS particle preparation section $(\S \mathrm{A}) .1 \mathrm{~mL}$ aliquots where transferred to $1.5 \mathrm{~mL}$ microcentrifuge tubes and separated using high speed centrifugation as per section $\mathrm{C}(\S \mathrm{C})$. The supernatant was discarded and the aliquots were resuspended in either D.I. water, PBS ( $\mathrm{pH} 7)$, sodium citrate buffer ( $\mathrm{pH} 5)$ or PBS ( $\mathrm{pH}$ 2). The CS particle sizes were measured at $\mathrm{t}=0,2,4,8,24,48,96,168,336$ and 672 hours using the same procedure as described in a section $\mathrm{B}(\S \mathrm{B})$. In between time points the samples were stored in an incubator (VWI International, West Chester, PA) set at $37^{\circ} \mathrm{C}$. 


\section{2. $\mathrm{pH}$ Back Titration Study}

A pH back titration study was completed because in previous swelling studies CS particles swelled in both PBS ( $\mathrm{pH} 7)$ and sodium citrate buffer $(\mathrm{pH}$ 5); therefore, it was important to determine if the reaction was reversible or if there was a point when no swelling occurred. In this study, CS particles where fabricated from $0.1 \% \mathrm{TPP} \mathrm{pH} 4.5$ and $0.125 \% \mathrm{CS}_{250}$ solution as described in section $\mathrm{A}(\S \mathrm{A})$. The CS particles where centrifuged (§C). The supernatant was then discarded and replaced with PBS (pH 7), PBS (pH8) or sodium citrate buffer ( $\mathrm{pH} 5)$. Their size was measured at hour $0(\S \mathrm{B})$. For the resuspended CS particles in PBS (pH 7) the $\mathrm{pH}$ of their buffer was adjusted to $\mathrm{pH} 5$ with either $.01 \mathrm{~N}$ HCL or sodium citrate buffer. For the other resuspended CS particles in PBS (pH 7), the $\mathrm{pH}$ of their buffer was adjusted to $\mathrm{pH} 8$ with either $.01 \mathrm{NaOH}$ or PBS (pH 8). The $\mathrm{pH}$ was constantly measured using a pH meter (Denver Instruments, Germany). Finally, after the $\mathrm{pH}$ adjustments the sizes were again measured $(\S \mathrm{B})$.

\section{H. BSA Encapsulation in Chitosan Particles}

To study and optimize the encapsulation of a drug in the CS particles, bovine serum albumin "BSA" (Equitech Bio Inc., Kerrville, TN) was used as a model drug. BSA was used based on its cost effectiveness. BSA was dissolved directly into the $0.5 \%$ LMW CS solution at concentrations of $0.25,0.5,1$ and $2 \mathrm{mg} / \mathrm{mL}$ BSA. CS particles were then fabricated as described in section A $(\S \mathrm{A})$. It is important to note that the $0.5 \% \mathrm{TPP}$ solution was adjusted to $\mathrm{pH}$ 4.5. The crosslinker genipin was also added after CS particle fabrication at a $0.5 \mathrm{mM}$ concentration. For comparison CS particles without BSA were fabricated. The CS particles were separated by centrifugation (§C). $100 \mathrm{uL}$ of the supernatant was pipetted into microcuvettes and measured at a wavelength of $280 \mathrm{~nm}$ by 
spectrophotometer (Beckman Coulter Du 530, Brea, CA), to determine the encapsulation efficiency. BSA concentrations were determined from a standard curve of known BSA concentrations versus absorption. To calculate encapsulation efficiency the following equation (Equation 1) was used.

$$
\text { Encapsulation Efficiency }=\frac{\text { Total Amt. BSA }- \text { Free Amt. BSA }}{\text { Total Amt. BSA }} * 100(\text { Equation } 1)
$$

BSA concentration was also measured using a bicinchoninic "BCA" assay (Sigma Aldrich, St. Louis, MI) to calculate BSA encapsulation efficiency. The same encapsulation efficiency protocol was followed; however, $150 \mu \mathrm{L}$ of the supernatant was transferred to a 96 well plate. The assay protocol was completed as recommended by the vendor. It is important to note that the assay works by correlating protein concentration to a colormetric change produced by BCA binding to $\mathrm{Cu}^{1+}$ under alkaline conditions. $\mathrm{Cu}^{1+}$ is produced by reduction of $\mathrm{Cu}^{2+}$ by cysteine, cystine, tryptophan, tyrosine and peptide bonds of the protein of interest. The 96 well plated was placed in an incubator for 2 hours at $37{ }^{\circ} \mathrm{C}$ before measuring the absorbance at $562 \mathrm{~nm}$ with a plate reader (BioTek ELX800, Winooski, VT).

Encapsulation was also visualized using confocal microscopy. Fluorescent microscopy was not used because the resolution was too low to see CS particles properly. In order, to view CS particles via confocal microscopy they were encapsulated with BSA conjugated to fluorescein isothiocyanate "FITC" (Invitrogen, Eugene, OR). To use the 
BSA conjugated to FITC, $5 \mathrm{mg}$ BSA+FITC was dissolved in $2 \mathrm{~mL}$ D.I. water. HMW CS particles were then fabricated using $0.5 \%$ TPP pH 4.5 and $0.5 \%$ HMW CS solutions, which were then crosslinked with $0.5 \mathrm{mM}$ genipin. It is important to note that the BSA was encapsulated by two different methods. The first method involved mixing the 0.125 $\mathrm{mg} / \mathrm{mL} \mathrm{BSA+FITC}$ with the CS solution before adding the TPP or genipin. The second method involved allowing the $0.125 \mathrm{mg} / \mathrm{mL}$ BSA+FITC to diffuse into the CS particles after fabrication. After the CS particles were fabricated they were allowed to dry on a glass cover slip overnight. The CS particles were also imagined when wet. Imagines were captured during a Zeiss confocal microscopy demonstration.

\section{BSA Release from CS Particles}

\section{Low Molecular Weight Chitosan Particles}

Before BSA release studies were completed, swelling studies of CS particles encapsulating BSA was completed. This was done to help better explain the release kinetics of BSA during release studies. $0.125 \mathrm{mg} / \mathrm{mL}$ BSA was encapsulated into LMW CS particles as described above for encapsulation efficiency studies (§G). For comparison, CS particles without BSA were also made. Next, $1 \mathrm{~mL}$ samples from CS particle solutions were centrifuged $(\S \mathrm{C})$. The supernatant was removed and replaced with either PBS (pH 7) or sodium citrate buffer ( $\mathrm{pH}$ 5). Lastly, their size was measured over time at $\mathrm{t}=0,1$ and 4 hours.

$1 \mathrm{mg} / \mathrm{mL}$ BSA encapsulated particles were prepared as described above for encapsulation efficiency studies $(\S G)$. After the initial $100 \mu$ l of supernatant was removed for encapsulation efficiency studies, the remainder was discarded and the CS particles were resuspended in D.I. water, PBS ( $\mathrm{pH} 7)$ or sodium citrate $(\mathrm{pH}$ 5). At $\mathrm{t}=0,1,2,4$ and 
8 hours, $100 \mu \mathrm{L}$ of the samples were removed and its absorbance at $280 \mathrm{~nm}$ was measured by spectrophotometer. For all suspensions, the blank was appropriately matched to the suspension buffer. In between time points, the samples should be kept in an incubator at $37^{\circ} \mathrm{C}$ and agitated occasionally to prevent the CS particles from settling on the bottom of the microcentrifuge tubes.

The sensitivity of the spectrophotometer is limited and cannot accurately measure the low BSA release concentrations. Therefore, BSA conjugated to FITC was used; instead, of just BSA. A fluorometer (Turner Biosystems, Sunnyvale, CA) was used to measure the amount of BSA release. CS particles were fabricated from $0.5 \% \mathrm{TPP} \mathrm{pH} 4.5$ and $0.5 \%$ CS solutions ( $\S \mathrm{A})$, which were crosslinked with $1 \mathrm{mM}$ genipin. A $100 \mu \mathrm{L}$ of the dissolved BSA-FITC solution was then added to $1 \mathrm{~mL} \mathrm{CS}$ particle solution for a final concentration of $0.25 \mathrm{mg} / \mathrm{mL}$. The CS particles were then continuously mixed overnight on a rotating plate. Next, the CS particles were separated by centrifugation ( $§ \mathrm{C}) .200 \mu \mathrm{L}$ of the supernatant was removed and measured with the fluorometer (460/515-570 wavelengths) to calculate the encapsulation efficiency. The remaining supernatant was discarded and replaced with either PBS ( $\mathrm{pH} 7$ ) or sodium citrate buffer ( $\mathrm{pH} 5)$. Fluorometric readings of $200 \mu \mathrm{l}$ samples (which were replaced with the appropriate buffer) were then taken at $\mathrm{t}=0,1,2,4$ and 8 hours. In between time points sampled were placed in an incubator at $37^{\circ} \mathrm{C}$. Measurements were compared to a standard curve of known concentrations of BSA-FITC.

Release studies of BSA from particles were also performed to determine the effect of continuous mixing. CS particles were created following the protocol described above. $50 \mu \mathrm{L}$ of the BSA-FITC solution was added to a $1 \mathrm{~mL}$ aliquot of CS particles in $1.5 \mathrm{~mL}$ 
centrifuge tubes for a final BSA concentration $0.125 \mathrm{mg} / \mathrm{mL}$. The CS particles were continuously mixed on a rotating plate for 24 hours. The BSA concentration was lowered from 0.25 to $0.125 \mathrm{mg} / \mathrm{mL}$ BSA-FITC based on over-saturation that was occurring in the previous procedure. Next, the CS particles where centrifuged (§C). After centrifugation, $50 \mu \mathrm{L}$ of the supernatant was diluted in $50 \mu \mathrm{L}$ of D.I. water and measured using a fluorometer. The rest of the supernatant was discarded and the CS particles were resuspended in PBS ( $\mathrm{pH} 7$ ) or sodium citrate buffer ( $\mathrm{pH}$ 5). The samples were then transferred to a 48 well plate and mixed on a magnetic stir plate. At $0,1,2,4$ and 8 hours $100 \mu \mathrm{L}$ samples (which were replaced with the appropriate buffer) were removed to be measured using a fluorometer.

\section{Heppe Medical Chitosan Particles}

The swelling studies were again repeated for the Heppe Medical Chitosan. CS particles were fabricated from $0.1 \% \mathrm{TPP} \mathrm{pH} 4.5$ and $0.125 \% \mathrm{CS}(250 \mathrm{kDa})$ solutions. The BSA was encapsulated following two different methods. In the first method, $250 \mu \mathrm{L}$ BSA was added to the CS solution before CS particle formation. In the second method, $250 \mu \mathrm{L}$ BSA was added after CS particle formation. Both resulted in a final BSA concentration of $.125 \mathrm{mg} / \mathrm{mL}$. After formation, both types of BSA encapsulated CS particles were placed on a rotating mixer for 24 hours. The size of the CS particles was measured before centrifugation (§C). The supernatant was then removed and the CS particles were resuspended in either 1mL PBS (pH 7) or sodium citrate buffer ( $\mathrm{pH} 5)$. Size measurements for the supernatant; as well as, the resuspended CS particles at $\mathrm{t}=0,1$ and 4 hours were taken measured $(\S \mathrm{B})$. In between time intervals, CS particles were placed in an incubator. It is also important to note that CS particles were redispersed by 
pipetting up and down several times before every size measurement. For further procedural step clarification see Figure 11.

BSA Solution: Dissolve BSA in D.I. water at a 2.5 $\mathrm{mg} / \mathrm{mL}$ concentration. Used for both methods.

Method 1 (Before): $0.125 \%$ Heppe Medical CS dissolved in D.I. water with an acetic acid concentration 1.75 times that of the CS concentration. Mix 0.125 $\mathrm{mg} / \mathrm{mL}$ BSA into CS solution.
Method 2 (After): $0.125 \%$ Heppe Medical CS dissolved in D.I. water with an acetic acid concentration 1.75 times that of the CS concentration.

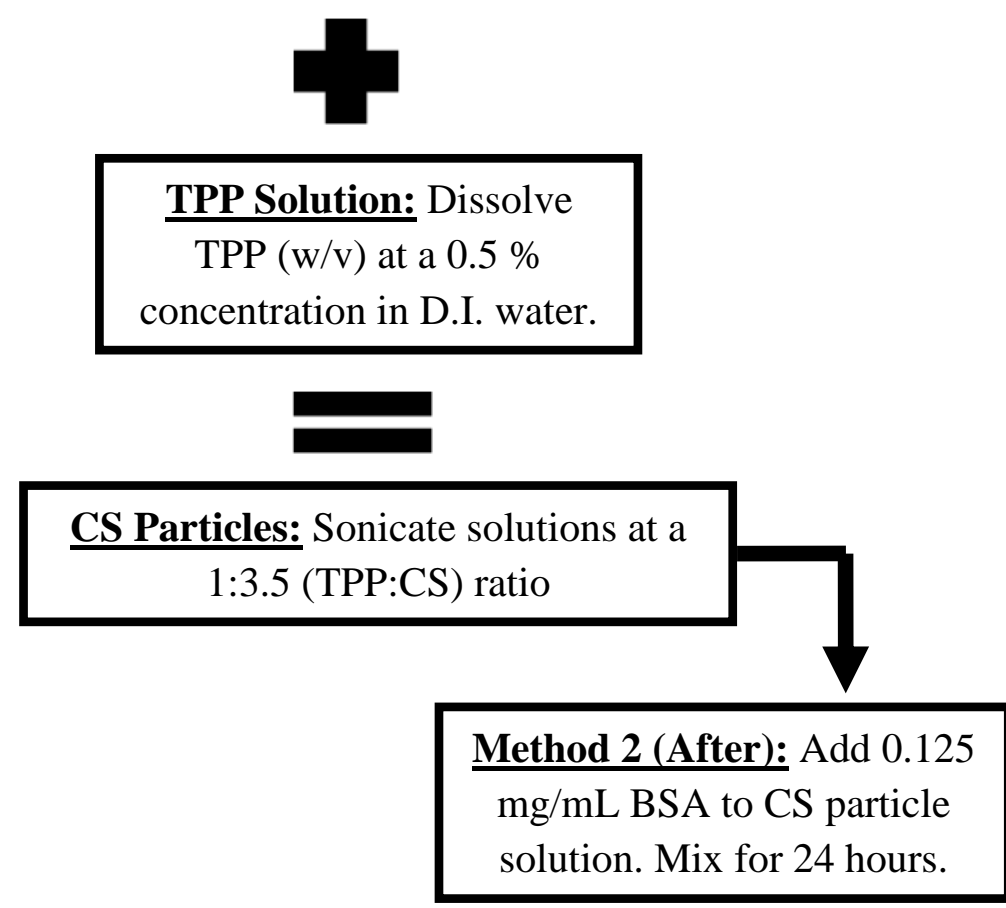

Figure 11 - BSA Before and After Chitosan Particle Encapsulation Methods It is important to note that release of BSA from Heppe Medical Chitosan particles was completed using a BCA assay; however, the sodium citrate interfered with the assay causing erroneous results. Therefore, they will not be presented in this paper even though some release was seen in PBS (pH 7) (data not shown). 


\section{J. NAGase Release From Chitosan Particles}

The release study was done to show the efficacy of CS particles as a protein carrier for NAGase (Sigma Aldrich, St. Louis, MI). $0.390 \mathrm{~mL}$ of NAGase $(1 \mathrm{mg} / \mathrm{mL})$ was dialyzed in D.I. water for 12 hours using a slide-A-Lyzer Mini Dialysis unit with a MWCO cutoff of $3.5 \mathrm{kDa}$ (Thermo Scientific, Rockford, IL). It is important to note that the $0.390 \mathrm{~mL}$ was divided into separate microdialyer wells. Dialysis was important for removing the ammonium sulfate; in which, the NAGase is dissolved in since it causes the chitosan solution to aggregate. In order, to determine the concentration of the amount of NAGase recovered, the dialyzed solution's weight was measured and it was assumed that $1 \mu \mathrm{L}=1 \mathrm{mg}$. From there $0.240 \mathrm{mg}$ of the NAGase recovered was mixed into $5 \mathrm{~mL}$ of the $0.125 \%$ CS Heppe Medical solution. The rest of the NAGase was retained for controls and the standard curve. The CS particles were composed of $0.1 \%$ TPP pH 4.5 and 0.125 $\% \mathrm{CS}_{250}$ solution (§A). CS particles without NAGase were also fabricated as a control. Each CS particle sample was then divided into $1.5 \mathrm{~mL}$ microcentrifuge tubes and centrifuged as described in section $\mathrm{C}(\S \mathrm{C})$. The supernatant was then removed and saved. The CS particles were then resuspended in $1 \mathrm{~mL}$ of PBS (pH 7) or sodium citrate buffer (pH 5). Each sample was than divided into $100 \mu \mathrm{L}$ and placed within a 96 well plate for an $\mathrm{n}=5$ with each well correlating to a time point of $0,1,2,4$ and 8 hours. The concentration of NAGase of the supernatant and at samples at different time points was determined with a NAGase activity kit (Sigma Aldrich, St. Louis MI). The assay protocol was completed as recommended by the manufacturer for a 96 well plate. The NAGase activity kit is based on the hydrolysis of 4-Nitrophenyl N-acetyl- $\beta$-D-glucosaminide (NPGlcNAc) by NAGase. The enzymatic hydrolysis of the substrate releases p-nitrophenol, 
which upon ionization in basic $\mathrm{pH}$ can be measured colorimetrically at $405 \mathrm{~nm}$. It is important to note that studies were done to confirm that the activity of NAGase after dialysis was not changed (data not shown). In order to correlate activity to a NAGase concentration, a standard curve was employed. It is important to note that a standard curve was carried out for NAGase suspended in PBS (pH 7) and sodium citrate (pH 5).

\section{K. Statistical Analysis}

For statistical analysis of the size, zeta potential, centrifugation, swelling, encapsulation and release studies, Microsoft Excel and Minitab were used to find averages and standard deviations. The software packages were also used to create graphs. For comparisons among different parameters and studies t-tests and ANOVA was utilized with a p-value of less than 0.05 being significant. Finally, for imaging studies Image $\mathbf{J}$ (NIH) was utilized to determine size and polydispersity. 


\section{RESULTS AND DISCUSSION}

The following sections detail the results and discussion of the different procedures performed to characterize and optimize the CS particles as a drug delivery system for a biofilm degrading enzyme, NAGase. This is important because biofilms are one of the main causes of antibiotic resistance in CRBSIs, which results in increased mortality and morbidity of critically ill patients. It was proven that CS particles can be fabricated within a nano-sized range through DLS and SEM; therefore, making them optimal to penetrate the biofilm for effective delivery of NAGase. The associated zeta potential was also found to be positive, which confirms the mucoadhesive properties of chitosan. Thereby, potentially helping to increase the residence time of the CS particles within the biofilm. High speed centrifugation was also determined as the best method of CS particle separation for use in other studies. However, the centrifugation procedure did show the poor mechanical properties of the CS particles as described in the literature. Therefore, different crosslinkers, such as sodium citrate, GPTMS and genipin, were experimented with to try to increase the CS particles mechanical strength. It was also later found that reducing the $\mathrm{pH}$ of the TPP solution to 4.5 helped decrease the size and polydispersity of the CS particles; therefore, increasing reproducibility. Next, the $\mathrm{pH}$ dependent swelling properties of the CS particles were confirmed with the lower $\mathrm{pH}$ resulting in faster swell 
rates. Finally, encapsulation and release of BSA and NAGase from the CS particles was confirmed; however, the values were often low. Therefore, more optimization and characterization is needed. Taking all the results into consideration, CS particles exhibit a good potential as a drug delivery system to degrade biofilms for treatment of CRBSIs.

\section{A. Fabrication of Chitosan Particles}

It was found that through the ionic gelation method, CS particles were fabricated instantaneously. This was due to the ionic bonds forming between the amino groups of the chitosan and the negatively charged phosphates of the TPP. Figure 12 below represents a gross imagine of Heppe Medical CS particles taken by SEM. The size and polydispersity will be discussed later.

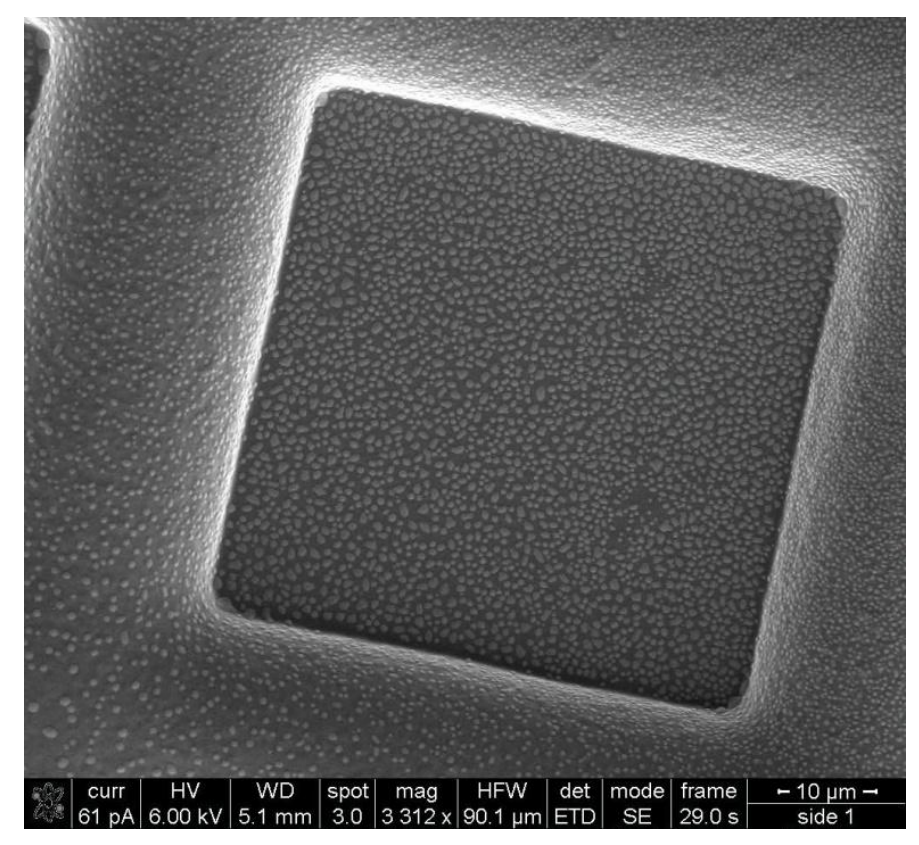

Figure 12 - SEM Image of Heppe Medical Chitosan Particles

\section{Size Measurement of Chitosan Particles}

a. Low Molecular Weight Chitosan Particles. CS and TPP concentration were varied; in order, to determine the optimal range needed to fabricate CS particles with 
desired nanometer diameter features. From the literature, it was seen that different concentrations of CS and TPP determined the sizes of CS particles fabricated (Calvo, Remunan, J.L.V., \& Alonso, 1997). It was observed that the smallest average diameter, $236.9 \pm 38.8 \mathrm{~nm}$, was obtained from the concentrations of $0.25 \%$ (w/v) CS and $0.25 \%$ (w/v) TPP (Figure 13 and Table II). As CS concentration increased, higher TPP concentrations were needed to produce smaller CS particles. However, if too high of a TPP concentration was used, larger average diameters $(0.25 \%$ and $0.5 \% \mathrm{CS})$ resulted, most likely due to the repulsion of charges since there are more cations available. The 1 $\%$ CS was the exception for there appeared to be little variation in average diameter no matter the TPP concentration. The smallest particles for $1 \% \mathrm{CS}$ were found at $1 \% \mathrm{TPP}$, $1239.3 \pm 419.9 \mathrm{~nm}$. The last important thing to notice was that the variation in data was smaller for lower CS and TPP concentrations (Figure 13).

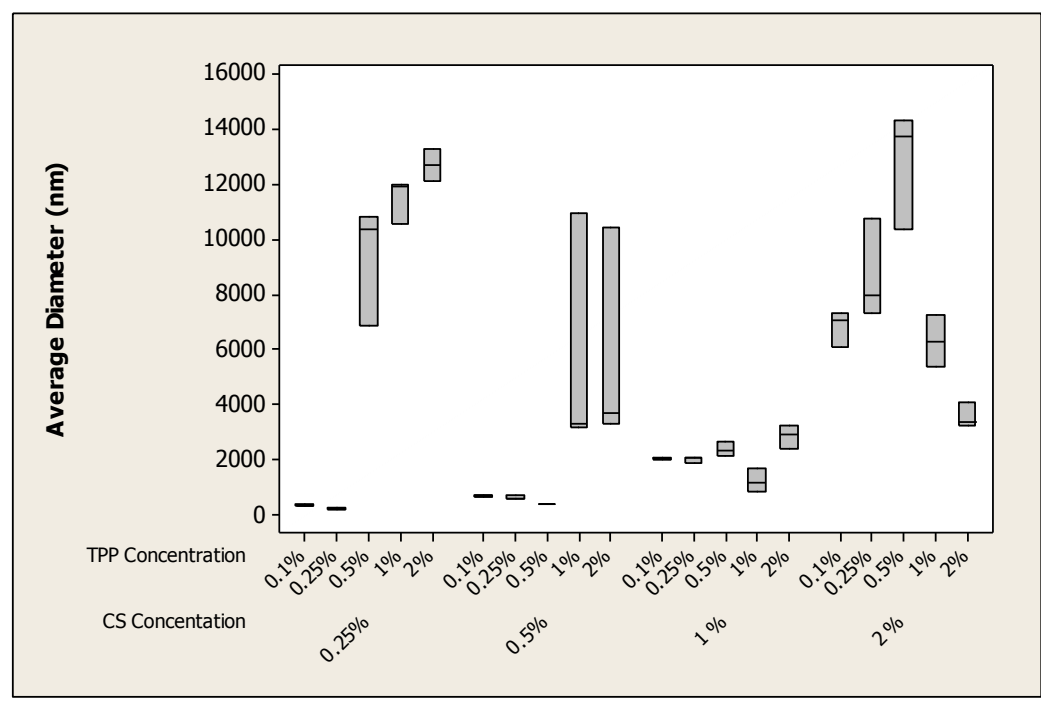

Figure 13 - Size of Chitosan Particles Based on Varying Concentrations (w/v) of LMW CS and TPP

TABLE II AVERAGE SIZE OF CS-TPP PARTICLES (nm) LMW CS Concentration 


\begin{tabular}{|c|c|c|c|c|} 
TPP Concentration & $0.25 \%$ & $0.50 \%$ & $1 \%$ & $2 \%$ \\
\hline $0.10 \%$ & $335.8 \pm 41.8$ & $695.7 \pm 20.2$ & $2049.3 \pm 38.8$ & $6805.7 \pm 626.3$ \\
\hline $0.25 \%$ & $236.9 \pm 38.8$ & $635.1 \pm 79.8$ & $2011.3 \pm 133.9$ & $8640 \pm 1812$ \\
\hline $0.50 \%$ & $9317 \pm 2164.2$ & $404.5 \pm 13.2$ & $2376 \pm 237.8$ & $12770 \pm 2130.3$ \\
\hline $1 \%$ & $11456.7 \pm 820.4$ & $5790 \pm 4434.3$ & $1239.3 \pm 419.9$ & $6291 \pm 903.3$ \\
\hline $2 \%$ & $12668.9 \pm 571.9$ & $5813.7 \pm 4002.5$ & $2847.7 \pm 436.2$ & $3556.3 \pm 467.77$ \\
\hline
\end{tabular}

The study was repeated to further evaluate the correlation of LMW CS and TPP concentrations; in addition, to particle size. The study was specifically repeated for CS and TPP concentrations between $0.25-0.5 \% \mathrm{w} / \mathrm{v}$ and 0.1 and $0.5 \% \mathrm{w} / \mathrm{v}$, respectively (Table III). The data showed that the studies were not statistically different, as the p-value was always greater than 0.05 .

\begin{tabular}{|c|c|c|c|}
\hline \multicolumn{4}{|c|}{ TABLE III } \\
\hline \multicolumn{2}{|c|}{ COMPARISON OF AVERAGE DIAMETER OF LMW CHITOSAN PARTICLES } \\
CS \% & TPP \% & $\begin{array}{c}\text { First Study } \\
\text { Size (nm) }\end{array}$ & Second Study Size (nm) \\
\hline 0.25 & 0.1 & $311.8 \pm 26.1$ & $335.8 \pm 41.8$ \\
\hline 0.25 & 0.25 & $186.2 \pm 9.8$ & $236.9 \pm 38.8$ \\
\hline 0.5 & 0.1 & $640.1 \pm 34.1$ & $695.7 \pm 20.2$ \\
\hline 0.5 & 0.25 & $540.3 \pm 37.5$ & $635.1 \pm 79.8$ \\
\hline 0.5 & 0.5 & $311.5 \pm 35.4$ & $404.5 \pm 13.2$ \\
\hline 1 & 1 & $997.6 \pm 117.9$ & $1239.3 \pm 419.9$ \\
\hline
\end{tabular}

It was determined the final concentrations would be $0.5 \%$ LMW CS and $0.5 \%$ TPP for all subsequent studies due to its smaller CS particle size, smaller CS particle size deviation and theoretical ability to encapsulate more NAGase due to longer chitosan chains (Table III). The average diameters were also less than $1 \mu \mathrm{m}, 311.5 \pm 35.4$ and $404.5 \pm 13.2$, meaning they should be able to diffuse through the fluid filled channels of the biofilms (Drury, Stewart, \& Characklis, 1993). As mentioned in the introduction, this is extremely important to treat CRBSIs, since the bacteria that cause these infections produce a biofilm, which acts as a protective matrix against antibiotics. Therefore, if 
these CS particles could not enter the channels they would not be able to specifically deliver the enzyme to degrade the biofilms.

b. High Molecular Weight Chitosan Particles. In order to encapsulate a protein, it is assumed that a higher molecular weight chitosan would be needed, since there should be more linear CS chain length for the protein to get trapped in. Therefore, an additional study was completed to determine the concentrations for HMW CS and TPP concentrations needed to fabricate optimal sized particles (Figure 14 and Table IV). However, the CS particle diameters were far greater than in the previous studies where LMW CS was used. An explanation for the high particle size was that the chitosan was forming fibers, in addition to CS particles, as observed under light microscopy. Therefore, either the concentration of TPP needed to be increased/decreased or the molecular weight of the chitosan decreased in order to have better ionic interactions. To have more control over the diameter, chitosan produced within narrower manufacturing guidelines, such as deacetylation degree and molecular weight was purchased for further studies.

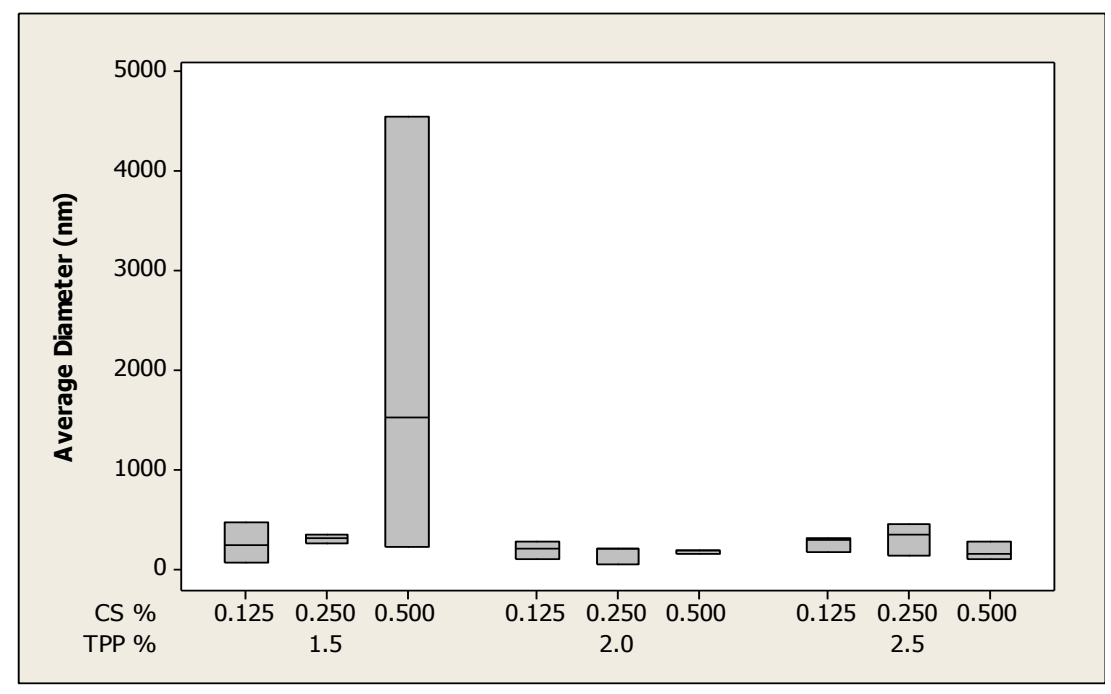


Figure 14 - Size of Chitosan Particles Based on Varying Concentrations (w/v) of HMW CS and TPP

\begin{tabular}{|c|c|c|c|}
\hline \multicolumn{4}{|c|}{ TABLE IV } \\
\hline \multicolumn{4}{|c|}{ HMW CS Concentration } \\
\hline APERAGE SIZE OF HMW CS-TPP PARTICLES (NM) \\
\hline & \multicolumn{3}{|c|}{$.5 \%$} \\
\hline $1.5 \%$ & $250.7 \pm 206.3$ & $304.9 \pm 44.1$ & $2099.9 \pm 2217.6$ \\
\hline $2 \%$ & $189.6 \pm 81.7$ & $143.7 \pm 83.2$ & $163.7 \pm 20.8$ \\
\hline $2.5 \%$ & $249.5 \pm 77.1$ & $305.7 \pm 157.3$ & $168.9 \pm 92.3$ \\
\hline
\end{tabular}

c. Heppe Medical Chitosan Particles. Three different parameters were analyzed; in order, to determine the optimal production zone of CS particles. The three parameters included: molecular weight of chitosan $\left(\mathrm{CS}_{150}, \mathrm{CS}_{250}, \mathrm{CS}_{350} \mathrm{kDa}\right), \mathrm{CS}$ concentrations $(0.125,0.25,0.5$ and $1 \%)$ and TPP concentration $(0.1,0.250 .5,1.0$ and $1.5 \%)$. It was observed that the $1 \% \mathrm{CS}_{250}$ and $\mathrm{CS}_{350}$ was too viscous and when mixed with TPP it automatically caused the chitosan to aggregate. Therefore, these values are not included in the following figures. As can be seen in the chart below the average diameter was again very large as compared to the HMW CS particles. CS fibers were again confirmed 
via light microscopy.

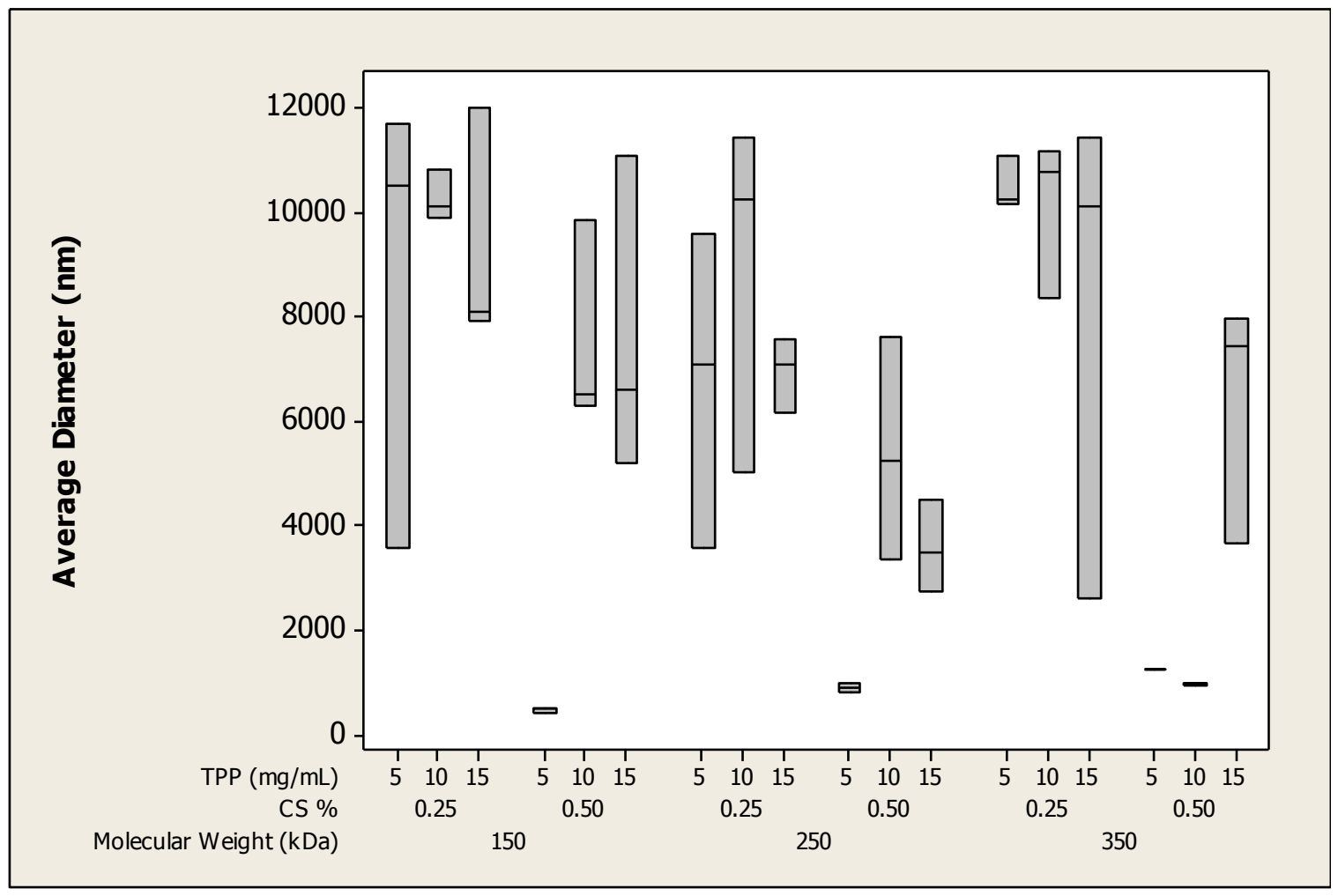

Figure 15 - Size of Chitosan Particles Based on Varying Concentrations (w/v) of Heppe

\section{Medical CS and TPP}

Based on this data, further studies were conducted, which included a $0.125 \%$ CS

solution at the varying molecular weights and a 0.1 or $0.25 \%$ TPP solution. For all formulations with TPP concentrations equal to $0.25 \%$, it was observed that chitosan fibers were still being formed via light microscopy. As the TPP concentration decreased, the number and size of the fibers also decreased. However, CS particles without fibers was seen when composed of a $0.1 \% \mathrm{TPP}$ and $0.125 \%, \mathrm{CS}_{250}$ solutions. These CS particles had an average diameter of 198 to $226 \mathrm{~nm}$ (Table V). In addition it was observed that these particles had a moderate polydispersity of $0.262-0.475$, which is typical of natural made polymers. 


\begin{tabular}{|c|c|}
\hline \multicolumn{2}{|c|}{ TABLE V } \\
\hline \multicolumn{2}{|c|}{ AVERAGE SIZE FOR $0.125 \% \mathrm{CS}+0.1 \%$ TPP } \\
\hline $\mathrm{MW}(\mathrm{kDa})$ & Size $(\mathrm{nm})$ \\
\hline $\mathrm{CS}_{150}$ & $198.5 \pm 13.3$ \\
\hline $\mathrm{CS}_{250}$ & $212.1 \pm 32.2$ \\
\hline $\mathrm{CS}_{350}$ & $226.1 \pm 20.3$ \\
\hline
\end{tabular}

When analyzing the size distribution of the CS particles among the different molecular weights the smallest variation in CS particle diameters was found to be the lower molecular weight $\mathrm{CS}_{150}$ (Figure 16). However, the earlier issues about the ability to encapsulate protein are still pertinent. Therefore, the $\mathrm{CS}_{250}$ was used in future studies, since the average diameter was not statistically different from other CS molecular weights.

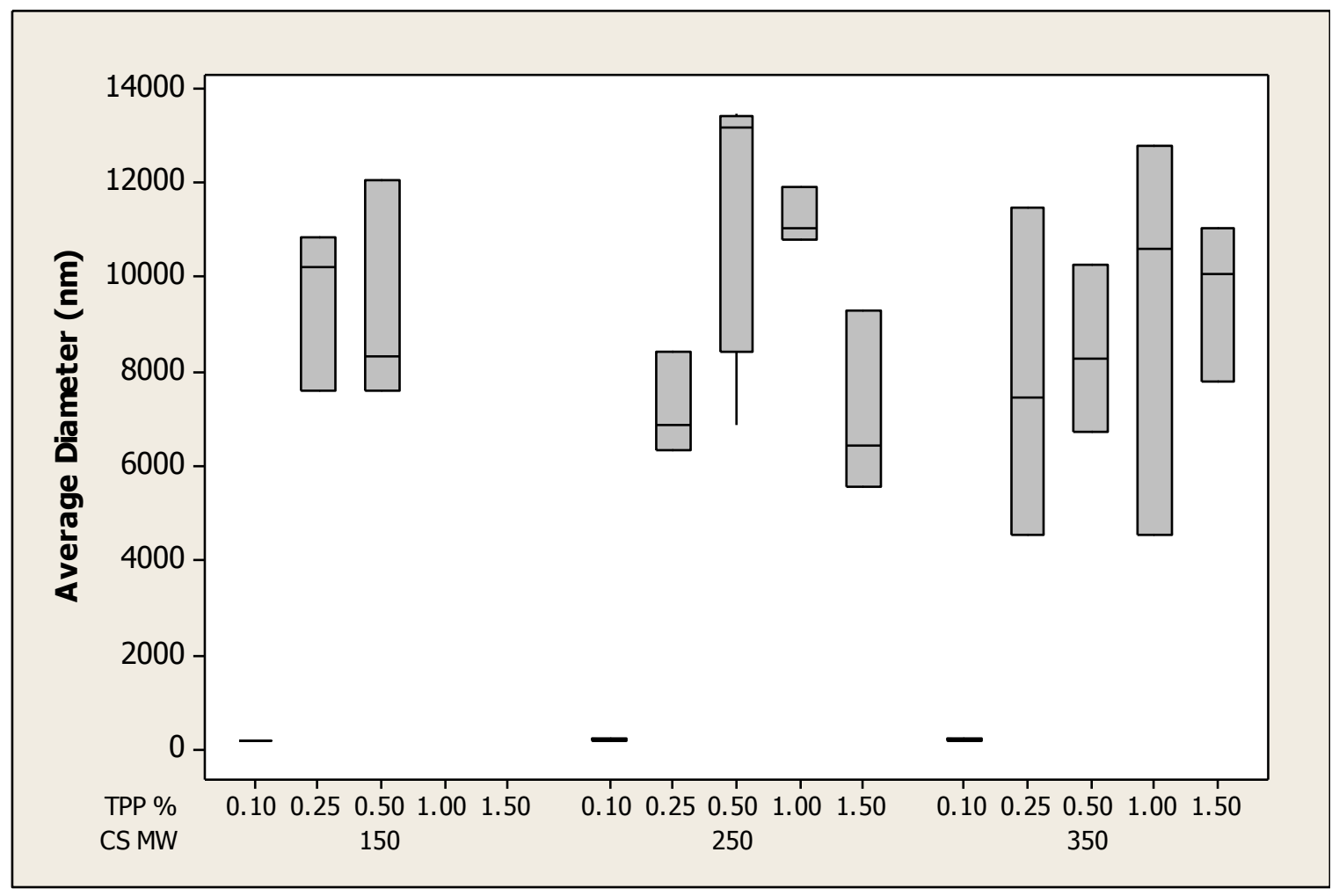

Figure 16 - Size of Chitosan Particles Based on Varying Concentrations (w/v) of Heppe Medical $0.125 \%$ CS and TPP 


\section{d. Different pH TPP Solution Effect on Chitosan Particle Size. The pH of the}

TPP solution was reduced in later studies based on literature that stated the CS particle size would decrease. By adjusting the $\mathrm{pH}$ of the TPP solution before mixing with LMW CS, one can modulate the concentration of anions which in turn can influence the interaction between the TPP and CS. By adjusting the $\mathrm{pH}$ between 4 and 9 (native concentration of TPP in water), it was observed that the average size of the CS particles formed can change. It is important to note that the original $\mathrm{pH}$ of the TPP solution was approximately $\mathrm{pH}$ 9. As seen in Figure 17, the TPP solution adjusted to $\mathrm{pH} 4.5$ exhibited the smallest average diameter CS particle $(318.0 \pm 32.7 \mathrm{~nm})$ with little variability.

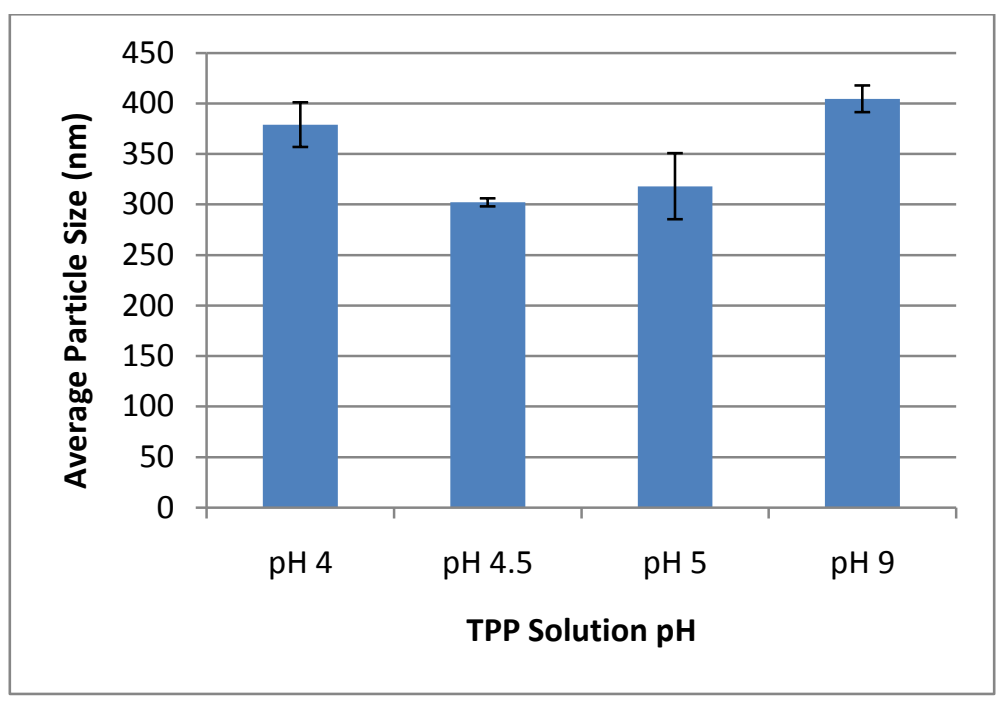

Figure 17 - Average Size of Chitosan Particles Based on Varying pH of TPP Solution

A lower TPP solution $\mathrm{pH}$ needs to be maintained, since at higher $\mathrm{pH}$ 's the size starts to increase. This is due to the competing hydroxyl and phosphoric ions that are trying to interact with the amine sites of the chitosan. But at lower $\mathrm{pH}$ 's the hydrogen ions are either interacting with the phosphoric ions or repelling the amine sites of chitosan. Hence, at a certain midpoint the $\mathrm{pH}$ will have an optimal amount of ions from 
the TPP to interact with the amine sites of the chitosan to form CS particles. It is expected that the Heppe Medical Chitosan particles will act in a similar manor since the same chemistry is still being utilized.

\section{Zeta Potential Measurement of Chitosan Particles}

The zeta potential measurement is important for several reasons. First it confirms the mucoadhesive properties of chitosan. This is important because the residence time of the CS particles is increased at the biofilm; therefore, it is possible for longer NAGase release to occur. Secondly, knowing the charge can also help determine how the NAGase or BSA is interacting with the CS particles during encapsulation and release studies.

Thirdly, knowing the charge can help explain swelling characteristics, especially when placed in buffers with different $\mathrm{pH}$ 's. Fourthly, the charge can affect the interaction between CS particles. For example, the more negatively or positively charged the CS particles are then the more they will repel each other. Finally, the zeta potential can help determine the stability of the CS particles.

a. Low Molecular Weight Chitosan Particles. The zeta-potential was measured in conjugation with the LMW CS size study at time point 0 . The results are shown in Table VI.

\begin{tabular}{|c|c|c|c|c|}
\hline \multicolumn{5}{|c|}{ TABLE VI } \\
\hline ZETA POTENTIAL $(\mathrm{mV})$ OF LMW CS-TPP PARTICLES \\
\hline & \multicolumn{5}{c|}{ LMW CS Concentration } \\
\hline TPP Concentration & $0.25 \%$ & $0.50 \%$ & $1 \%$ & $2 \%$ \\
\hline $0.10 \%$ & $37.8 \pm 1.1$ & $38.4 \pm 2.1$ & $35.4 \pm 5.4$ & $34.3 \pm 6.5$ \\
\hline $0.25 \%$ & $20.33 \pm .7$ & $37.93 \pm 2.5$ & $38.47 \pm 1.7$ & $31.5 \pm 6$ \\
\hline $0.50 \%$ & $5.86 \pm .5$ & $19.7 \pm .5$ & $37.5 \pm 1.6$ & $19.93 \pm 6.8$ \\
\hline $1 \%$ & $2.91 \pm .1$ & $7.37 \pm .6$ & $25.47 \pm 2$ & $-7.71 \pm 10.1$ \\
\hline $2 \%$ & $3.21 \pm 6.4$ & $6.64 \pm 7.4$ & $-2.02 \pm .9$ & $-3.3 \pm 1.4$ \\
\hline
\end{tabular}


The results show that as the concentration of TPP increases the zeta potential decreases. As the concentration of CS increases the zeta potential increases. Meaning, that both trends are a result of there being either more or less negatively charged, phosphate groups, of the TPP or positively charged, amino groups, of the CS particles. Hence, at certain TPP and CS concentrations there will be complete ionic interactions; however, if either concentration is increased or decreased there will be a charge excess. This is better shown in the interaction plot of Figure 18.

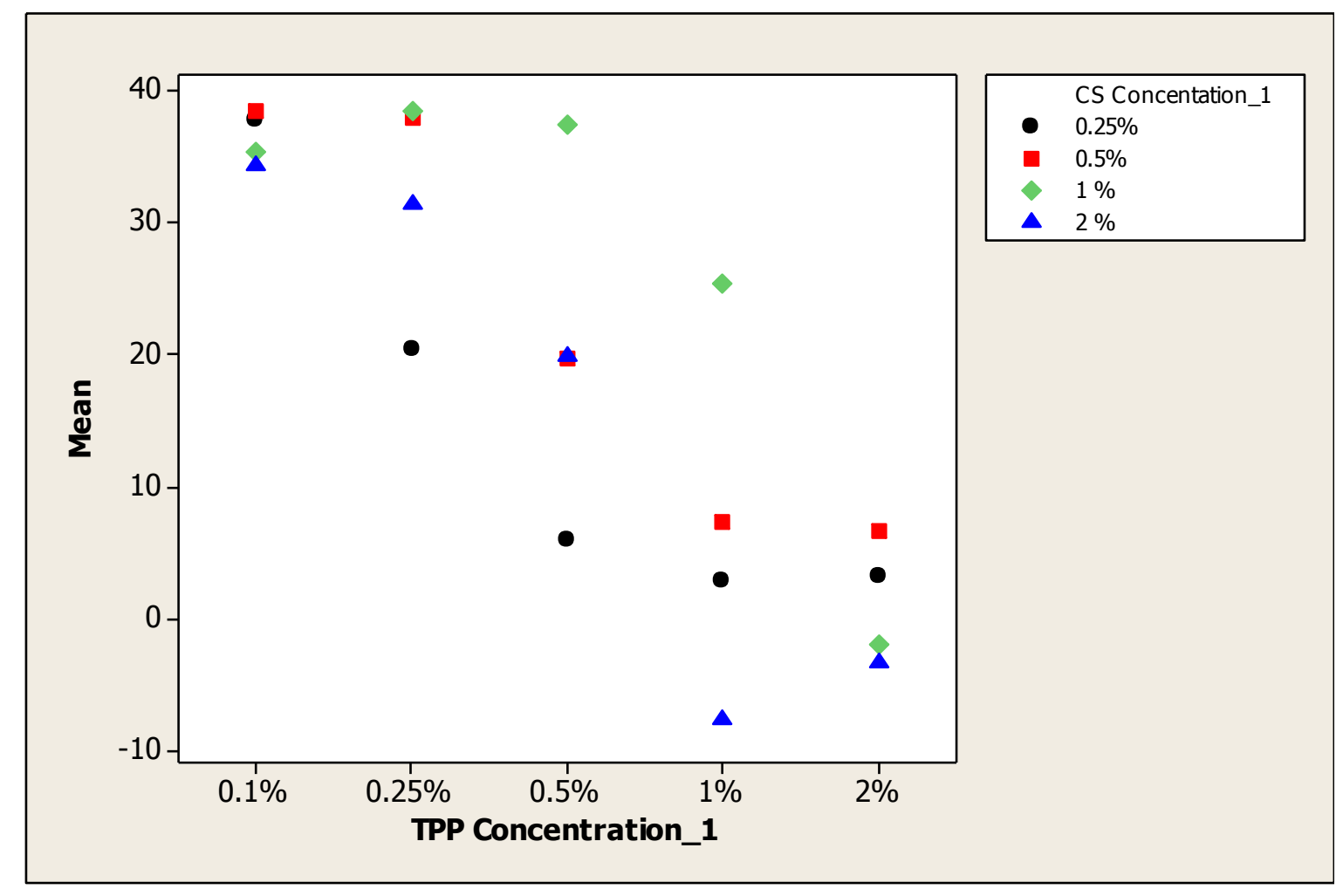

Figure 18 - Zeta Potential at Varying Concentrations of Chitosan and TPP

In conjugation with the previous study, zeta potential was also found during the second size study. The table below shows both studies had similar zeta potentials with no significant statistical difference. The zeta potential for the chosen optimal, CS particles composed of $0.5 \%$ TPP and $0.5 \%$ LMW CS solutions was between 19.7-22 mV. 


\begin{tabular}{|c|c|c|c|}
\hline \multicolumn{4}{|c|}{ TABLE VII } \\
\multicolumn{4}{|c|}{ AVERAGE ZETA POTENTIAL $(\mathrm{mV})$ OF CHITOSAN PARTICLE COMPARISON } \\
\hline CS $\%$ & TPP $\%$ & First Study Size $\left(\mathrm{d}^{*} \mathrm{~nm}\right)$ & Second Study Size $\left(\mathrm{d}^{*} \mathrm{~nm}\right)$ \\
\hline 0.25 & 0.1 & $37.8 \pm 1.1$ & $37.5 \pm 3.9$ \\
\hline 0.25 & 0.25 & $20.3 \pm 0.7$ & $19.9 \pm 1.1$ \\
\hline 0.5 & 0.1 & $38.4 \pm 2.1$ & $39.9 \pm 1.3$ \\
\hline 0.5 & 0.25 & $37.9 \pm 2.5$ & $37.8 \pm 1.0$ \\
\hline 0.5 & 0.5 & $19.7 \pm 0.5$ & $21.7 \pm 1.3$ \\
\hline 1 & 1 & $25.5 \pm 1.0$ & $33.4 \pm 1.7$ \\
\hline
\end{tabular}

Lastly, the zeta potential was recorded overtime during the first swelling study. This was important to establish the stability of the CS particles overtime. It was observed that while in buffered solution the zeta potential of the particles did not change overtime $(p>0.05)$ (Table VIII). However, there was significant difference $(p=0.000-0.014)$ in D.I. water between Day 1 and 10, Day 1 and 29 and Day 10 and 29. This difference could be because DI water was not buffered; therefore, the components can disassociate readily hence changing greatly the zeta potential overtime. Also it is important to note that at $\mathrm{pH}$ 5 the zeta potential is close to neutral $\mathrm{pH}$ since the buffer is more likely reaching the $\mathrm{CS}$ particles isoelectric point.

\begin{tabular}{|c|c|c|c|c|}
\hline \multicolumn{5}{|c|}{ TABLE VIII } \\
\hline \multicolumn{5}{|c|}{ Time Point Taken } \\
\hline Solvent & Day 1 & Day 4 & Day 10 & Day 29 \\
\hline D.I. Water & $32.8 \pm .6$ & $18.6 \pm 7.8$ & $26.2 \pm 0.6$ & $27.7 \pm 0.4$ \\
\hline $\mathrm{pH} 7$ & $13.3 \pm 2.3$ & $13.2 \pm 1.1$ & $13.4 \pm 2.4$ & $14.5 \pm 1.9$ \\
\hline $\mathrm{pH} 5$ & $3.0 \pm 0.2$ & $2.9 \pm 0.7$ & $2.8 \pm 0.4$ & $2.9 \pm 0.1$ \\
\hline $\mathrm{pH} 2$ & $12.2 \pm 8.8$ & $15.4 \pm 2.4$ & $19.2 \pm 4.5$ & $18.2 \pm 1.8$ \\
\hline
\end{tabular}

b. Heppe Medical Chitosan Particles. The zeta potential for the CS particles made with Heppe Medical chitosan is listed in Table IX. It is important to note the CS particles were composed of $0.1 \% \mathrm{TPP} \mathrm{pH} 4.5$ and $0.125 \% \mathrm{CS}_{250}$ solutions. The average zeta 
potential was $33.9 \pm 2.8 \mathrm{mV}$, which was comparable to the LMW CS particles. The positive charge was expected due the higher concentration of CS as compared to the TPP being used; as well as, as the low pH environment that the CS particles are fabricated in.

\begin{tabular}{|c|c|}
\hline \multicolumn{2}{|c|}{ TABLE IX } \\
ZETA POTENTIAL HEPPE MEDICAL CHITOSAN PARTICLES \\
\hline Sample \# & Zeta Potential $(\mathrm{mV})$ \\
\hline 1 & 32.2 \\
\hline 2 & 32.3 \\
\hline 3 & 37.1 \\
\hline
\end{tabular}

The zeta potential measurements taken show a trend based on the concentration of CS and TPP used. For the main CS particles used the zeta potential was found to be positive. This will allow for the CS particles to be mucoadhesive. It was also found for the LMW CS particles that they were stable over time when placed in a buffered solution. It was also determined that the buffer's $\mathrm{pH}$ affects the zeta potential of the CS particles. Finally, knowing the charge should help provide insight as to how the NAGase and BSA interact with the CS particles in encapsulation and release studies.

\section{Determination of Chitosan Particle Separation Method}

Determining the best procedure for CS particle separation is critical for several reasons. The main reason is to remove them from the fluid they were fabricated in. This will be important so that they can be used for future studies in which they will need to be resuspended in different fluids. The solution they were fabricated in might also have unnecessary artifacts that could interfere with future tests such as assays or when drying the CS particles for SEM. Secondly, CS particle separation could be critical for future storage when they might need to be suspended in sucrose for freezer storage, etc. Thirdly, separation is important in order to concentrate the particles for a higher amount of them. 
a. Low Molecular Weight Chitosan Particles. Two different methods were tried for CS particle separation, including rotary evaporation (rotavap) and high speed centrifugation. The rotavap method showed no statistical difference between the size before and after the liquid has evaporated (Table X). However, due to the low volumes of CS particles normally produced (approximately $7 \mathrm{~mL}$ ), the set-up proved too complicated and the entire process was very time consuming. There were also a lot of steps between the dialyzing and actual rotavap procedure that make it highly possible there was a significant loss of CS particles.

\begin{tabular}{|c|c|c|c|}
\hline \multicolumn{4}{|c|}{ TABLE X } \\
\hline \multicolumn{3}{|c|}{ ROTAVAP LMW CHITOSAN PARTICLE AVRAGE DIAMETER (NM) } \\
\hline CS \% & TPP \% & Before Rotavap & After Rotavap \\
\hline 0.5 & 0.5 & $327.6 \pm 14.9$ & $302.1 \pm 3.9$ \\
\hline 0.25 & 0.25 & $216.5 \pm 12.1$ & $329.6 \pm 48.4$ \\
\hline
\end{tabular}

Therefore, high speed centrifugation was pursued. The original CS particle diameter size was $320.2 \pm 34 \mathrm{~nm}$. The results (Table XI) show that centrifugation at $18,510 \mathrm{x}$ g's for 30 minutes at $10^{\circ} \mathrm{C}$ was found to have the smallest diameter increase of approximately $20.7 \%$. It is also important to note that the CS particles were also centrifuged for 10 minutes but the time was too low for proper separation (data is not shown). It was determined that as centrifugation times and speeds increase, aggregation starts to occur as seen in CS particles centrifuged at 28,903, 39,830 and 51,430 x g's (Table XI). The aggregation is most likely a result of the poor mechanical properties of the CS particles. Therefore, future studies were completed that looked at different crosslinkers and changing $\mathrm{pH}$ 's of solutions to increase mechanical strength. Lastly, in Table XI it can be seen that CS particles were centrifuged with and without glycerin. The glycerin was used to try to prevent CS particle centrifugation. However, it was 
determined to have little effect as the size between both groups is not statistically different. For all studies performed with $0.5 \%$ TPP and $0.5 \%$ LMW CS, the centrifugation parameters will be $18510 \mathrm{x}$ g's for 30 minutes at $10{ }^{\circ} \mathrm{C}$.

\begin{tabular}{|c|c|c|}
\hline \multicolumn{3}{|c|}{ TABLE XI } \\
PARTICLE SIZE (NM) $0.5 \%$ CS-0.5 \% TPP FOR 30 MINUTES AT $10{ }^{\circ} \mathrm{C}$ \\
\hline Speed (g) & No Glycerin & Glycerin \\
\hline 1950 & 886.7 & 647.5 \\
\hline 8230 & $622.4 \pm 14.1$ & $642.2 \pm 47.9$ \\
\hline 12860 & 559.2 & 518.3 \\
\hline 18510 & $443 \pm 13.3$ & $468.3 \pm 7.4$ \\
\hline 28930 & $879.3 \pm 75.5$ & $1182.7 \pm 210.9$ \\
\hline 39830 & 939.5 & 719.4 \\
\hline 51430 & $3144.7 \pm 258$ & $3550 \pm 366.4$ \\
\hline
\end{tabular}

A change was made in the fabrication process, in which genipin was added to the CS particles as a second crosslinking agent to increase mechanical strength of the particles (Note: For additional information on the explanation of genipin use see Section $\mathrm{B}$ on different crosslinkers.) The $\mathrm{pH}$ of the TPP solution was also changed to 4.5 , to control the number of protonated ions on the TPP to fabricate smaller CS particles with a tighter polydispersity. It should be of note that studies just crosslinked with genipin and no change in $\mathrm{pH}$ of the TPP solution are shown in section B crosslinker studies. As mentioned previously, genipin was added to change the mechanical properties of the CS particles; therefore, the first centrifugation times and speeds were no longer acceptable. When the study was repeated it was found that the CS particles spun at 40,000 $\mathrm{x}$ g's for 30 minutes at $10{ }^{\circ} \mathrm{C}$ showed the smallest increase in CS particle diameter size as compared to the original diameter (See Table VII). It should be noted that LMW CS particles centrifuged at approximately the same time and speed had an increase in size of approximately $200 \mathrm{~nm}$ (See Table XI and Table XII). A trend is also shown for the 30 
minute centrifugation time, in that as centrifugation speeds increase the diameter increases. This trend is not apparent for the 60 minute centrifugation time. The increase in centrifugation speed was expected because the changes in the fabrication process helped to increase the surface mechanical strength with further crosslinking. Based on the following data all CS particles composed of a $0.5 \%$ TPP pH 4.5 and $0.5 \%$ LMW CS solutions crosslinked with $0.5-1 \mathrm{mM}$ genipin solution will be centrifuged at 40,000 x g's for 30 minutes at $10^{\circ} \mathrm{C}$.

\begin{tabular}{|c|c|c|c|}
\hline \multicolumn{4}{|c|}{ TABLE XII } \\
\hline $\begin{array}{c}\text { CEntrifugation Force } \\
(\mathrm{g} \text { 's) }\end{array}$ & Time (minutes) & $\begin{array}{c}\text { Average Size } \\
(\mathrm{d} * \mathrm{~nm})\end{array}$ & $\begin{array}{c}\text { \% Increase in Size } \\
\text { from Before } \\
\text { Centrifugation }\end{array}$ \\
\hline Before Centrifugation & 0 & $\mathbf{3 3 6 . 3} \pm \mathbf{2 2 . 3}$ & $\mathbf{0}$ \\
\hline 40000 & 30 & $572.5 \pm 49.5$ & 41.3 \\
\hline 20000 & 30 & $678.0 \pm 78.8$ & 50.4 \\
\hline 10000 & 30 & $794.9 \pm 155.6$ & 57.7 \\
\hline 40000 & 60 & $660.3 \pm 68.2$ & 49.1 \\
\hline 20000 & 60 & $611.1 \pm 72.4$ & 45 \\
\hline 10000 & 60 & $644.8 \pm 106.4$ & 47.8 \\
\hline
\end{tabular}

b. Heppe Medical Chitosan Particles. For the Heppe Medical Chitosan particles, composed of $0.1 \%$ TPP and $0.125 \% \mathrm{CS}_{250}$, a centrifugation study needed to be repeated. This was due to the different type of chitosan being used; as well as, CS and TPP concentrations. The following speeds and times were chosen based on previous studies. The initial size before centrifugation was $212.1 \pm 23.2 \mathrm{~nm}$ for 30 minutes and $262.3 \pm$ $42.6 \mathrm{~nm}$ for 60 minutes. It can be seen in Figure 19 that there was an increase in average size of the particles that were centrifuged for 30 minutes at all speeds. The same trend for the 60 minute time interval was followed until the centrifugation speed of $39,830 \mathrm{x}$ g's; however, due to error seen in the standard deviation the trend could still persist. It is also 
important to note that the CS particle size after centrifugation is within the range of the CS particle sizes before centrifugation. Based on this data all CS particles composed of Heppe Medical Chitosan at a $0.1 \%$ TPP and $0.125 \%$ CS solution will be centrifuged at $39,830 \mathrm{x}$ g's for 60 minutes at $10{ }^{\circ} \mathrm{C}$.

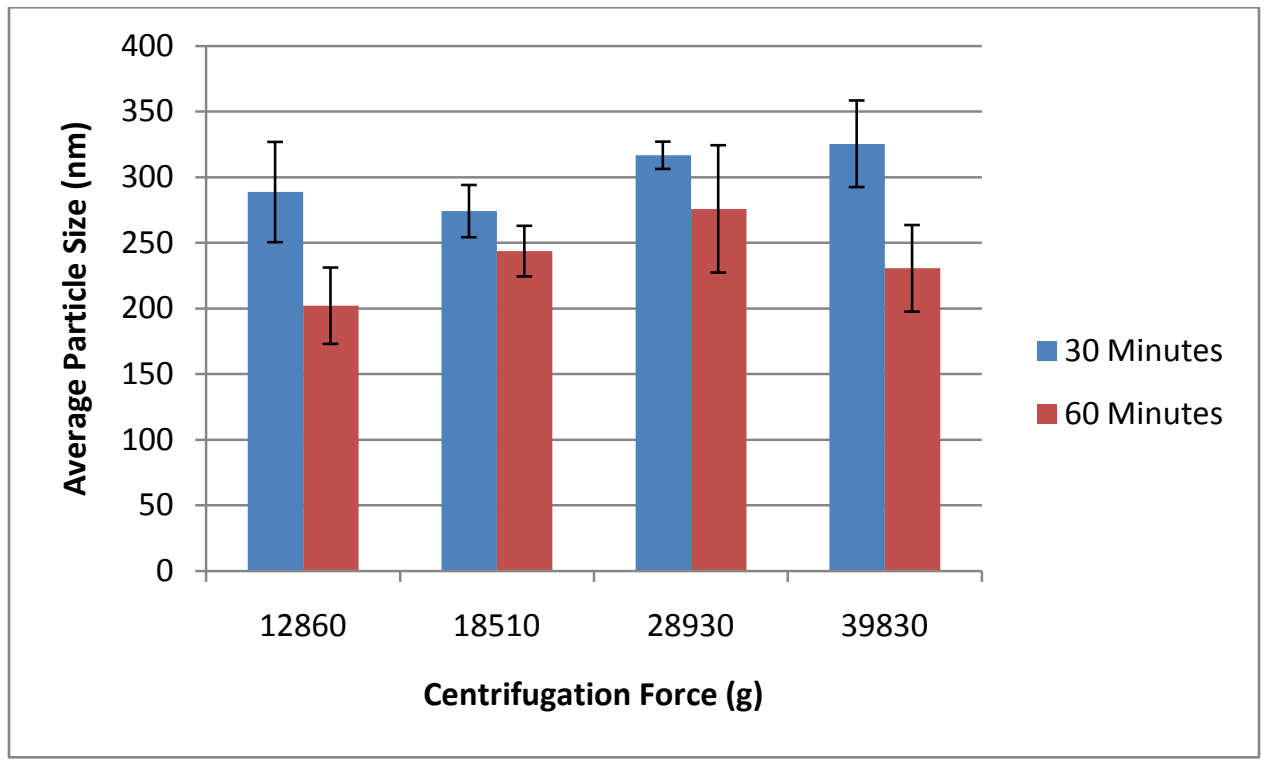

Figure 19 - Centrifugation Study for Heppe Medical Chitosan

As mentioned previously, the best method for CS particle separation was high speed centrifugation. However, times and speeds varied greatly, depending on the CS particle fabrication process. It is important to note that no one centrifugation time and speed resulted in ideal conditions. Meaning, not all of the CS particles were removed from the supernatant and the pellet of CS particles was not easily resuspendable if at all. The main limiting factor for not achieving ideal separation was due to the poor mechanical strength of the CS particles; therefore, new methods to look at increasing mechanical properties of the CS particles needs to be completed. However, it was proven that CS particles can be separated, which is extremely important for future swelling and release studies. 


\section{Morphological Characterization of Chitosan Particles}

a. Low Molecular Weight Chitosan Particles. Morphological features were characterized using SEM. The size of the LMW CS particles in the SEM images were measured using Image $\mathbf{J}$ and were compared to DLS techniques (Table XIII). It was seen that the diameters were of comparable diameter.

\begin{tabular}{|c|c|c|c|}
\hline \multicolumn{4}{|c|}{ TABLE XIII } \\
CHITOSAN PARTICLE SIZE COMPARISON BETWEEN SEM AND DLS \\
MEASUREMENTS \\
\hline CS \% & TPP \% & SEM Size (nm) & DLS Size (nm) \\
\hline 0.25 & 0.25 & 182.66 & 236.87 \\
\hline 0.5 & 0.5 & 328.47 & 404.5 \\
\hline 1 & 1 & 978.01 & 1239.3 \\
\hline
\end{tabular}

For the $0.25 \%$ TPP- $0.25 \%$ CS the particles formed larger non-spherical aggregates (Figure 20). These formations could be a result of there being more chitosan than TPP so they didn't fully crosslink. For the $0.5 \%$ TPP- $0.5 \%$ CS particles they are spherical and the size is more uniformly distributed (Figure 21). Lastly, for the $1 \%$ TPP$1 \% \mathrm{CS}$ particles there appeared to be a larger distribution of the diameters of CS particles; however, that could be due to the lower magnification (Figure 22). It is also important to note that there is a residue circling the dried CS particles, which is probably an artifact from the air drying process. 


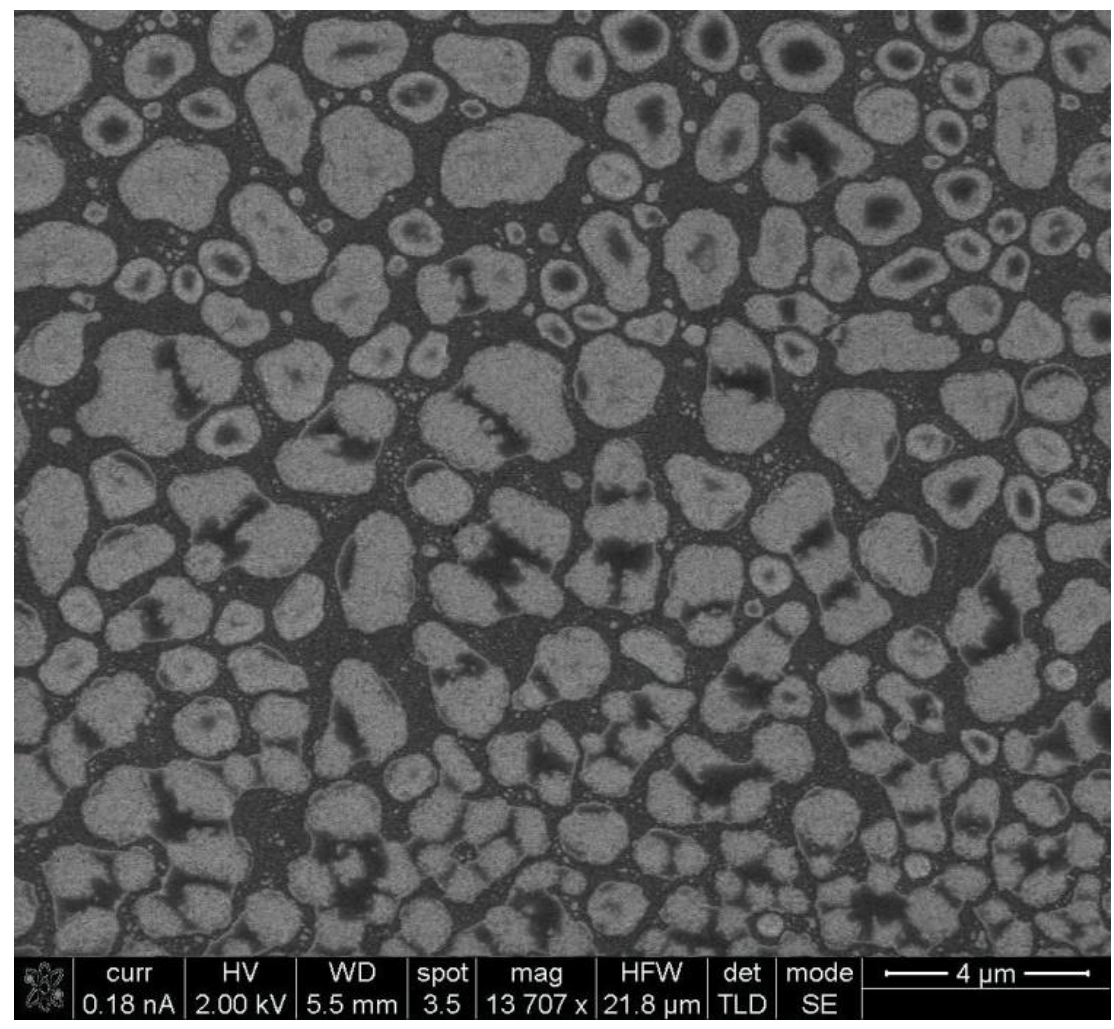

Figure 20 - SEM Image of Chitosan Particles (0.25 \% TPP-0.25 \% LMW CS)

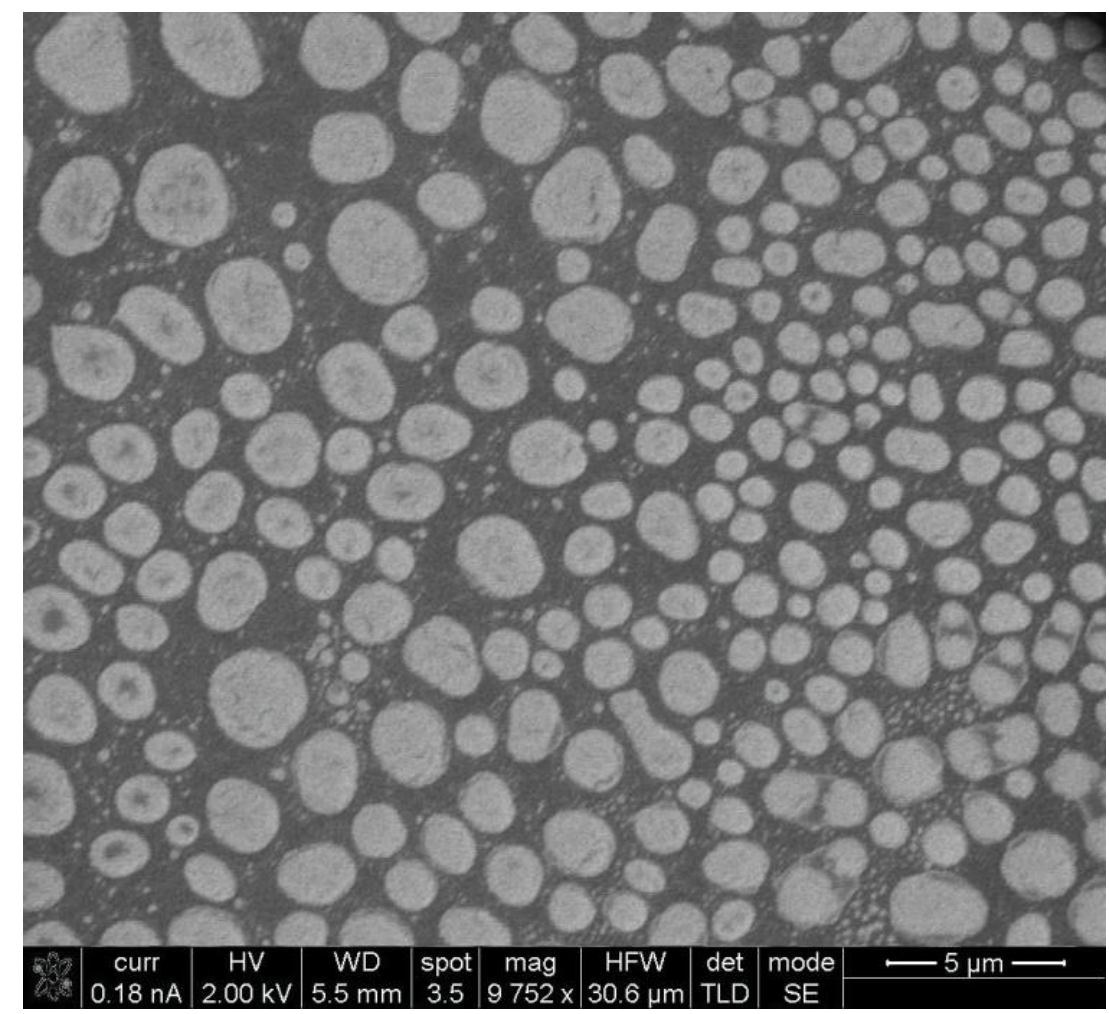

Figure 21 - SEM Image of CS Particles (0.5 \% TPP-0.5 \% LMW CS) 


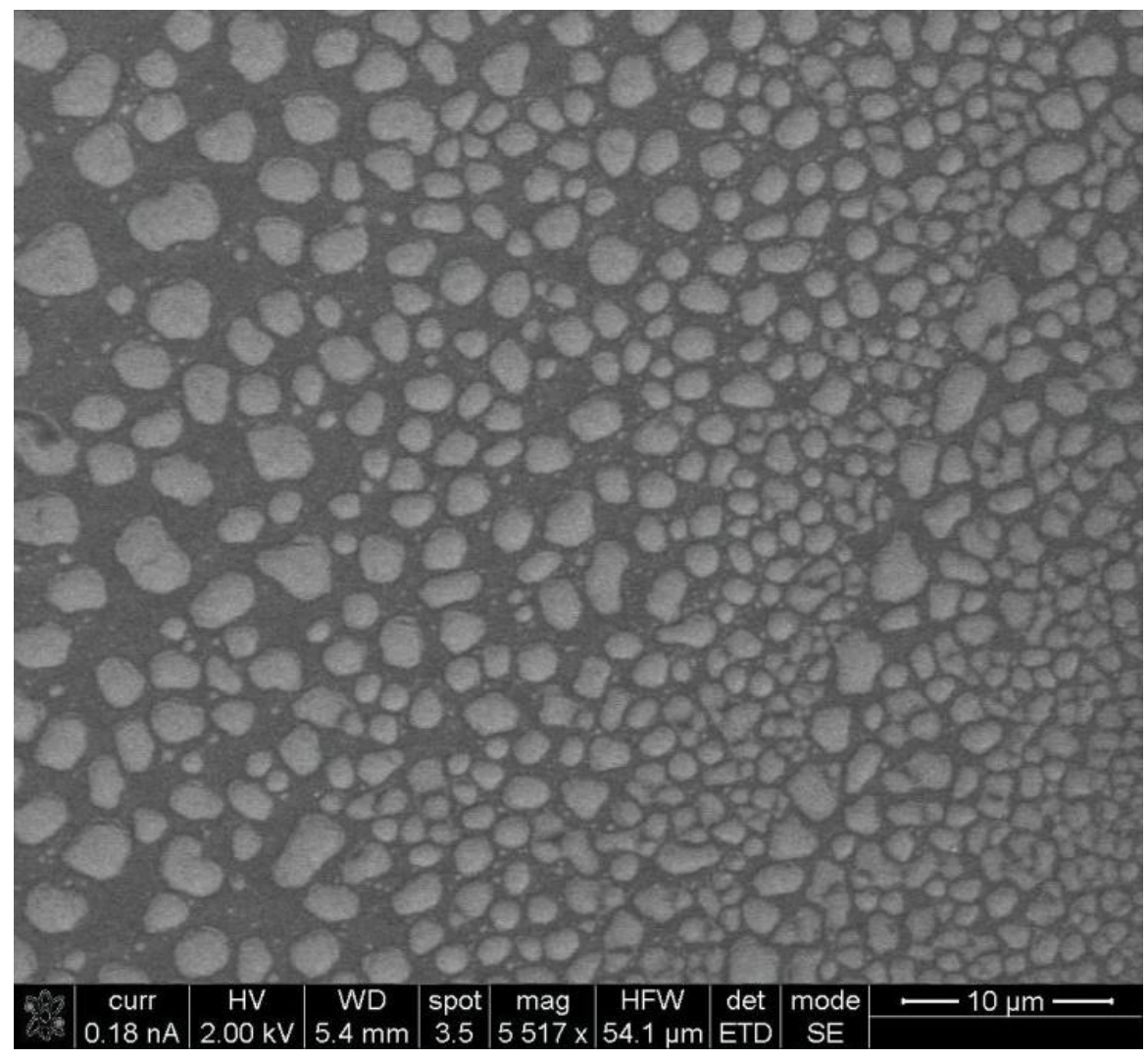

Figure 22 - SEM Image of CS Particles (1\% TPP-1 \% LMW CS)

b. Heppe Medical Chitosan Particles. To determine the morphological

characteristics of Heppe Medical Chitosan Particles, SEM imagines were again taken (Figure 23). However, this time a different microscope was utilized, which exhibited a better resolution of $2 \mu \mathrm{m}$. The average size, found manually using Image $\mathrm{J}$, from three different imagines was $332.8 \pm 52.9 \mathrm{~nm}$. This was comparable to the average size of $315.2 \mathrm{~nm}$ found via DLS. The polydispersity was also found to be $29.9 \pm 2.8 \%$, which is well within the acceptable range of natural polymers. Lastly, the images show that the CS particles are spherical. 


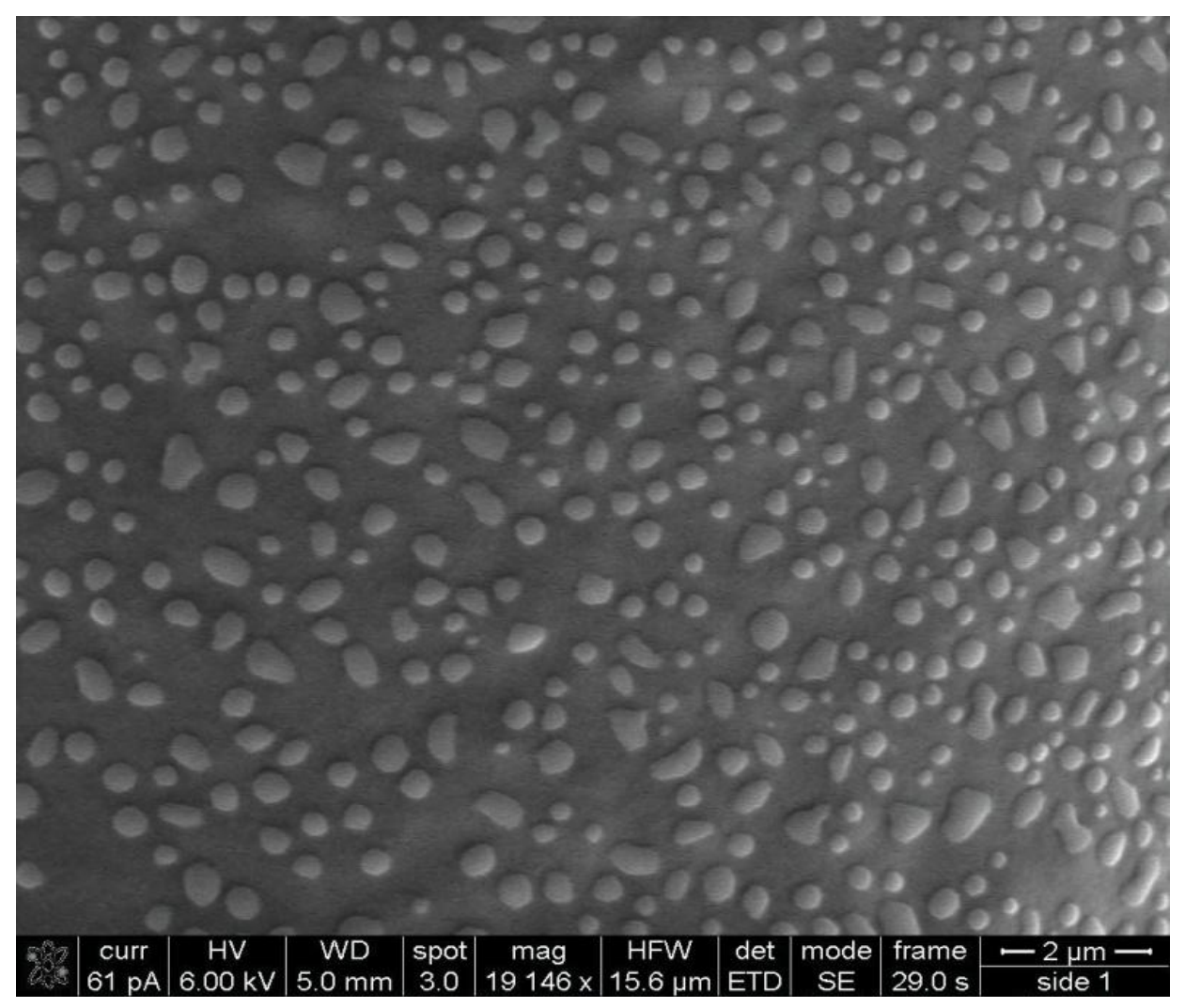

Figure 23 - SEM Image of Heppe Medical Chitosan Particles

\section{5. $\mathrm{pH}$ Swelling Study Effect on Chitosan Particles}

In order to describe the release profile of the encapsulated protein, swelling studies were performed. The desired profile includes a burst release in low $\mathrm{pH}(\mathrm{pH} 5)$ environments, but controlled release in neutral environments. The release is required at $\mathrm{pH} 5$ or lower due to the bioactivity of the enzyme of interest, NAGase. The release profiles were compared to buffered environments at $\mathrm{pH} 2$ and 7, as well as, in D.I. water.

a. Low Molecular Weight Chitosan Particles. The first swelling study involved LMW CS particles, which were placed in 3 different $\mathrm{pH}$ buffers and D.I. water as a control (See Figure 24). The CS particles size before centrifugation was $503.9 \mathrm{~nm}$ and after was $871.5 \pm 66.2 \mathrm{~nm}$. The control showed that there was no swelling over time and the diameters of the CS particles were similar to the diameters of those after 
centrifugation (Table XI). This is due to the similarity of the D.I. water to the solution that the CS particles were first fabricated in.

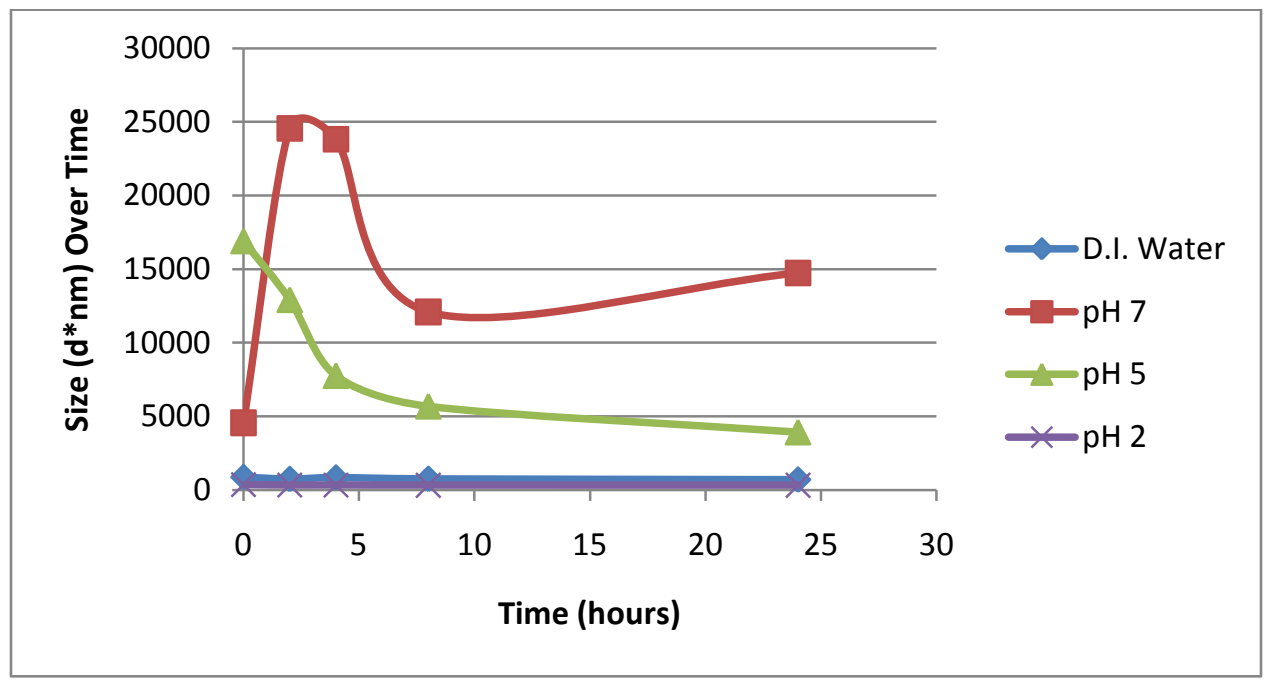

Figure 24 - Chitosan Particle Diameter During the First 24 Hours

The CS particles suspended in PBS ( $\mathrm{pH}$ 2) showed a noticeable decrease in CS particle diameter size at hour 0 , which remained constant over time. This significant size decrease was probably caused by the total dissolution of CS particles at time point 0 . The CS particles resuspended in sodium citrate $(\mathrm{pH} 5)$ showed an immediate increase in diameter, which slowly decreased over time eventually plateauing at around $3000 \mathrm{~nm}$. The CS particles resuspended in PBS (pH 7), initially swelled at $5000 \mathrm{~nm}$, followed by a dramatic increase to $23810.6 \mathrm{~nm}$ at hour 4 . However, within 24 hours the CS particles began to shrink and plateau around 48 hours to $3500 \mathrm{~nm}$ (Figure 25). 


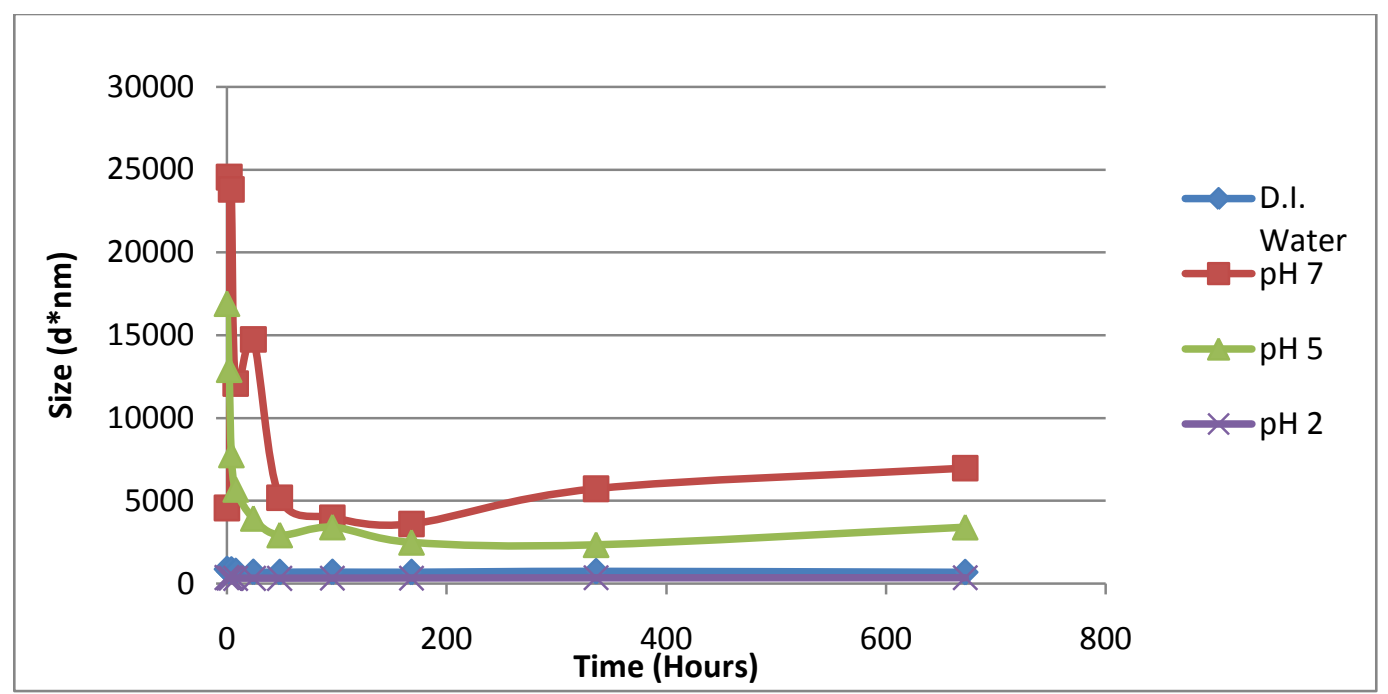

Figure 25 - Chitosan Particle Diameter Over 1 Month

b. $\mathrm{pH}$ Back Titration Study. For the $\mathrm{pH}$ back titration study it was found that CS particles swell rapidly in sodium citrate buffer ( $\mathrm{pH}$ 5), followed within 10 minutes by PBS ( $\mathrm{pH} 7)$. For the CS particles resuspended in PBS ( $\mathrm{pH} 8$ ), the size remained similar to the size of the CS particles measured before centrifugation, showing no swelling. When the CS particles are resuspended in PBS $(\mathrm{pH} 7)$ and then the $\mathrm{pH}$ is adjusted no reversible trend is seen. The CS particles whose solution was adjusted to $\mathrm{pH} 5$ do not show a similar increase in size as compared to the CS particles that were immediately resuspended in sodium citrate buffer ( $\mathrm{pH}$ 5). This may be due to the fact that the CS particles suspended in PBS (pH 7) have already undergone rapid swelling as seen in the change in size from 0 minutes to 10 minutes in Table XIV below. Therefore, the swelling has already greatly increased the surface area of the CS particles, thereby exposing their ionic bonds even more so that when the $\mathrm{pH}$ is adjusted lower, dissolution is actually occurring. Lastly, for the CS particles resuspended in PBS ( $\mathrm{pH} 7$ ) whose $\mathrm{pH}$ was adjusted to 8 showed that swelling that had already occurred is not reversible. However, it did appear that further swelling was prohibited in that the size did not reach the diameter of the CS particles 
whose $\mathrm{pH}$ was adjusted to 5 . It is important to note that this study was important to try to establish if the CS particles had an exact $\mathrm{pH}$ at which they swell or don't swell. It appears that it may be so but more future work at varying $\mathrm{pH}$ 's needs to be completed.

\begin{tabular}{|c|c|c|}
\hline \multicolumn{3}{|c|}{ TABLE XIV } \\
pH BACK TITRATION STUDY \\
\hline Name & Average $(\mathrm{nm})$ & Stdev $(\mathrm{nm})$ \\
\hline Before Centrifugation & 238.4 & 13.0 \\
\hline $\mathrm{pH}$ 5 resuspended & 4578 & 540.8 \\
\hline $\mathrm{pH} 7$ resuspended & 831.8 & 344.7 \\
\hline $\mathrm{pH}$ 8 resuspended & 231.9 & 33.4 \\
\hline $\mathrm{pH} 7$ adjusted to $\mathrm{pH} 5$ (0.01 N HCL) & 2011.33 & 244.1 \\
\hline $\mathrm{pH} 7$ Adjusted to $\mathrm{pH} 5 \mathrm{w} /$ Sodium Citrate & 2921 & N/A \\
\hline $\mathrm{pH} 7$ 10 minutes after resuspended & 3152 & 420.3 \\
\hline $\mathrm{pH} 7$ adjusted to $\mathrm{pH} 8$ (0.01 N NaOH) & 1356.5 & 191.6 \\
\hline
\end{tabular}

\section{B. Modulation of Chitosan Particles Via Crosslinking}

The addition of different crosslinkers was applied for two main reasons. The first reason was to try to reduce CS particle aggregation during centrifugation by increasing the mechanical strength with different crosslinkers and techniques. The second reason was to try to better control the swelling and protein release properties of the CS particles in different $\mathrm{pH}$ environments. Therefore, centrifugation and swelling studies were completed to determine how the different crosslinkers affected these CS particle characteristics.

\section{Trisodium Citrate}

The first additional crosslinker added was sodium citrate (Figure 26), which crosslinks through ionic interactions similar to TPP (Shu \& Zhu, 2002). 


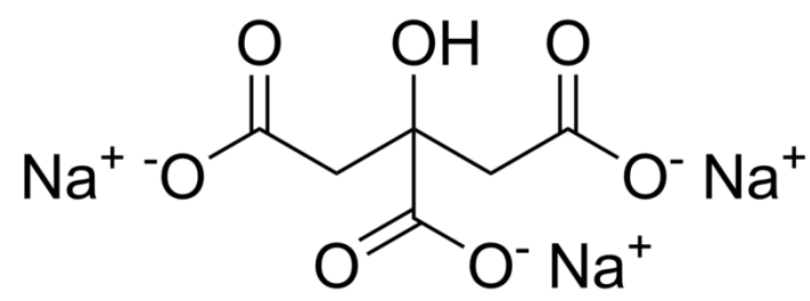

Figure 26 - Chemical Structure of Trisodium Citrate

The sodium citrate was mixed directly into the TPP solution at the same $0.5 \%$ concentration. The initial diameter before centrifugation of the CS particles fabricated only with TPP was $239.3 \mathrm{~nm}$. When sodium citrate was added the average diameter increased to $451.65 \pm 80.1 \mathrm{~nm}$. It is theorized the increase in diameter was due to further ionic crosslinking of still exposed positive chitosan amino groups.

A study on the swelling properties of CS particles crosslinked with the sodium citrate was completed immediately after fabrication and 24 hours later in solutions of various $\mathrm{pH}$. As seen in Table XV, there was an initial swelling at hour 0 for D.I. Water, pH 7 and pH 5 with a slow degradation at hour 24 (Table XV). It is also important to note that there is rapid degradation of CS particles in the low $\mathrm{pH}$ of PBS ( $\mathrm{pH} 2)$. Lastly, a general linear ANOVA showed a significant difference between the effect of buffers on swelling.

\begin{tabular}{|c|c|c|}
\hline \multicolumn{3}{|c|}{ TABLE XV } \\
SWELLING RESPONSE OF CHITOSAN PARTICLES CROSSLINKED WITH \\
SODIUM CITRATE \\
\hline Buffer & Hour 0 Size (nm) & Hour 24 Size (nm) \\
\hline D.I. Water & $2526.333 \pm 242.3$ & $1798.667 \pm 436.6$ \\
\hline pH 7 & $2013.333 \pm 855$ & $1972.333 \pm 793.2$ \\
\hline pH 5 & $2316.667 \pm 605.4$ & $1265.667 \pm 166.6$ \\
\hline pH 2 & $426.367 \pm 22.7$ & $369.600 \pm 17.7$ \\
\hline
\end{tabular}




\section{GPTMS}

In other studies, GPTMS (Figure 27) was also used as a covalent crosslinker because of its proven biocompatibility. It has also been used in other studies, in addition to TPP. In that study, it was proved that CS particles can still be fabricated using the same mild, spontaneous fabrication process, with retention of $\mathrm{pH}$ dependent swelling properties (Pan, $\mathrm{Wu}, \& \mathrm{Wu}, 2009)$.

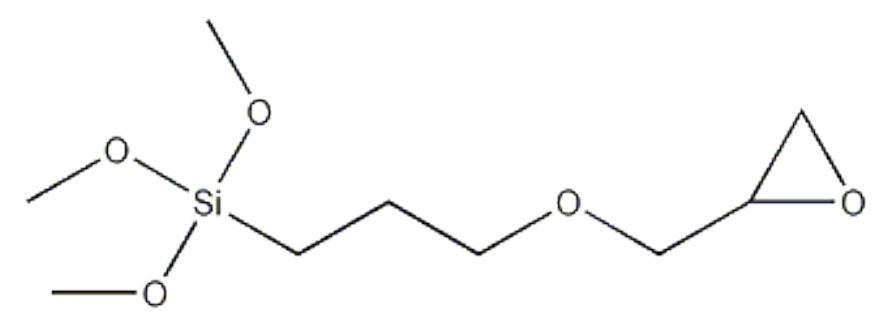

Figure 27 - Chemical Structure of GPTMS

In this study GPTMS was introduced during or after the addition of TPP to the CS solution. The initial size of CS particles when GPTMS was added after ionic gelation fabrication was $394.4 \pm 27.8 \mathrm{~nm}$. When GPTMS was added alongside the TPP the initial size of CS particles was $322.3 \pm 2.9 \mathrm{~nm}$. Compared to CS particles that were allowed to swell in D.I. water there was a marked increase in diameter in buffers at $\mathrm{pH} 7$ and $\mathrm{pH} 5$. As previously seen with the sodium citrate crosslinked CS particles, the GPTMS particles also instantly degraded in low $\mathrm{pH} 2$. As can be observed in Table XVI, there was a significant difference in swelling when comparing the timing of GPTMS addition (pvalue $=0.002)$. When GPTMS was added after ionic gelation there was a marked decrease in diameter (9-74\%) at 24 hours for particles in D.I. Water, $\mathrm{pH} 7$ and $\mathrm{pH} 5$ buffers. This could be a result of a two-fold swelling mechanism in that at hour 0 the TPP ionic interactions are controlling the swelling rate. But at 24 hours GPTMS is preventing further swelling since it crosslinks covalently. 


\begin{tabular}{|c|c|c|c|c|}
\hline \multicolumn{5}{|c|}{ TABLE XVI } \\
\hline \multicolumn{5}{|c|}{ CS PARTICLE DIAMETER (NM) CROSSLINKED WITH GPTMS } \\
\hline Buffer & Hour 0 & Hour 24 & Hour 0 & Hour 24 \\
\hline D.I. Water & $707.7 \pm 6.5$ & $604.3 \pm 8.5$ & $621 \pm 39.4$ & $298.8 \pm 8.5$ \\
\hline pH 7 & $1293.2 \pm 666.4$ & $2756 \pm 612$ & $2496.3 \pm 73.5$ & $1055.1 \pm 612$ \\
\hline pH 5 & $3119.7 \pm 1654.1$ & $2660.3 \pm 894.1$ & $4381.3 \pm 1273.7$ & $1123.7 \pm 894.1$ \\
\hline pH 2 & $362.7 \pm 1210.6$ & $292 \pm 22.4$ & $340.1 \pm 54.5$ & $371.9 \pm 22.4$ \\
\hline
\end{tabular}

\section{Genipin}

The last crosslinker tested was genipin (Figure 28). It was used because it has been proven in numerous literature reports to be 5,000-10,000 times less cytotoxic than glutaraldehyde (Sung, Huang, Huang, \& Tsai, 1999). It has also been used to make chitosan microparticles (Mi, Sung, Shyu, Su, \& Peng, 2003).

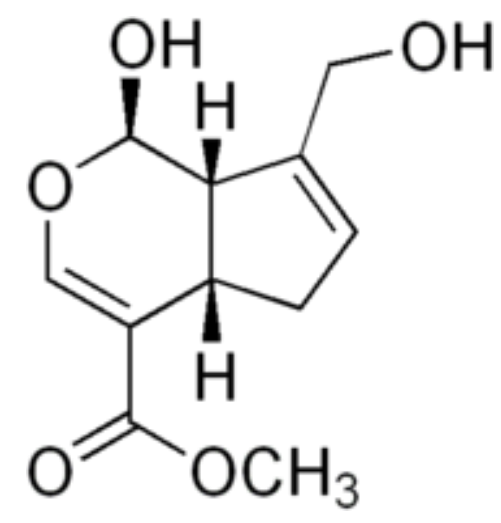

Figure 28 - Chemical Structure of Genipin

The genipin was also added either during or after ionic gelation of the chitosan to TPP. It is important to note that just genipin and chitosan solutions did not form CS particles. The initial size of the CS particles before centrifugation when genipin was added alongside TPP was $372.4 \pm 9.8 \mathrm{~nm}$ and when genipin was added after TPP was $326.8 \pm 74.3 \mathrm{~nm}$. The timing of when genipin was added was a significant factor in determining CS particle size ( $\mathrm{p}$-value $=0.008)$. When the genipin was added after ionic gelation there was rapid swelling in $\mathrm{pH} 7$ and $\mathrm{pH} 5$ buffers followed by continued 
swelling at hour 24. However, when the genipin was added before ionic gelation there was an initial swelling followed by degradation. The difference in swelling profiles is due to the method, in which genipin crosslinks the chitosan (Table XVII). For instance, when genipin is added after ionic gelation it is mainly crosslinking the outer surface of the particles; whereas, genipin added before ionic gelation is crosslinking the same amines as TPP, both the inner and outer diameter of the particles. Therefore, the CS particles, whose outer surface is crosslinked with genipin has an additional cage to take further swelling stresses. It is important to note that CS particles in low $\mathrm{pH}$ buffer $(\mathrm{pH} 2)$ were rapidly degraded at hour 0 , again following the same swelling trends noted in the sodium citrate and GPTMS studies.

\begin{tabular}{|c|c|c|c|c|}
\hline \multicolumn{5}{|c|}{ TABLE XVII } \\
\hline & Genipin After Particle Formation & Genipin During Particle Formation \\
\hline Buffer & Hour 0 $(\mathrm{nm})$ & Hour 24 $(\mathrm{nm})$ & Hour 0 $(\mathrm{nm})$ & Hour 24 $(\mathrm{nm})$ \\
\hline D.I. Water & $459.3 \pm 6.3$ & $392.4 \pm 8.3$ & $708.9 \pm 207.7$ & $599.3 \pm 150.6$ \\
\hline $\mathrm{pH} 7$ & $2279.3 \pm 548.4$ & $5938.3 \pm 279.7$ & $3124.7 \pm 1381.3$ & $2431.3 \pm 473.2$ \\
\hline $\mathrm{pH} \mathrm{5}$ & $3606.3 \pm 136.4$ & $5247.3 \pm 577$ & $2590 \pm 693$ & $1806 \pm 279.6$ \\
\hline $\mathrm{pH} 2$ & $332.3 \pm 22.6$ & $227.4 \pm 12.3$ & $431.8 \pm 62.1$ & $343.267 \pm 8.7$ \\
\hline
\end{tabular}

As previously shown, $\mathrm{pH}$ of the TPP solution can have an effect on the size of the CS particles formed. Hence, the size of CS particles were re-examined twice with the $\mathrm{pH}$ of the TPP solution adjusted to $\mathrm{pH} 4.5$ with genipin crosslinking. The study showed no significant difference $(\mathrm{p}$-value $=0.281)$ of CS particle size between experiments performed on different days (Table XVIII). 


\begin{tabular}{|c|c|c|c|c|}
\hline \multicolumn{5}{|c|}{ TABLE XVIII } \\
\hline & Genipin During Particle Formation & Genipin After Particle Formation \\
\hline & \multicolumn{3}{|c|}{ Hour 0 } & \multicolumn{2}{c|}{ Hour 0 } \\
\hline Name & Experiment 1 & Experiment 2 & Experiment 1 & Experiment 2 \\
\hline D.I. Water & $718.2 \pm 192.7$ & $726.6 \pm 46.5$ & $575.2 \pm 29.7$ & $616.4 \pm 18.1$ \\
\hline pH 7 & $251.5 \pm 12.9$ & $950 \pm 521$ & $1040.4 \pm 823.3$ & $858.5 \pm 446.5$ \\
\hline pH 5 & $2173.7 \pm 1334.3$ & $3244.3 \pm 618.5$ & $4596.7 \pm 687.8$ & $6259.3 \pm 1836.5$ \\
\hline & Hour 24 & \multicolumn{2}{c|}{ Hour 24 } \\
\hline & Experiment 1 & Experiment 2 & Experiment 1 & Experiment 2 \\
\hline D.I. Water & $591.1 \pm 155.3$ & $619.2 \pm 12.5$ & $416.1 \pm 35.4$ & $491.6 \pm 23.4$ \\
\hline pH 7 & $222.8 \pm 17.7$ & $1907.9 \pm 1645.2$ & $3857.7 \pm 836$ & $1760 \pm 915.6$ \\
\hline pH 5 & $5266.7 \pm 1111.5$ & $5243 \pm 1090.7$ & $3598.3 \pm 793.9$ & $5058.3 \pm 817.6$ \\
\hline
\end{tabular}

The table below (Table XIX) lists an overall view of the crosslinker studies for comparison. These characteristics included the original size before and after centrifugation; as well as, the swelling profile. From the table it was gathered that CS particles crosslinked with genipin after ionic gelation with TPP at $\mathrm{pH} 4.5$ provided the necessary CS particle characteristics. Meaning, CS particle's diameter was nano-sized. The CS particles also showed a higher swelling rate in $\mathrm{pH} 5$ than $\mathrm{pH} 7$ at hour 0 , which should better correlate to release of NAGase in the appropriate $\mathrm{pH}$ environment. 


\begin{tabular}{|c|c|c|c|c|c|c|c|c|}
\hline \multicolumn{9}{|c|}{$\begin{array}{c}\text { TABLE XIX } \\
\text { OVERALL CROSSLINKER STUDY }\end{array}$} \\
\hline \multicolumn{9}{|c|}{ Hour 0} \\
\hline Name & $\begin{array}{c}0.5 \% \\
\mathrm{CS}+ \\
0.5 \% \\
\mathrm{TPP} \\
\end{array}$ & $\begin{array}{c}0.5 \% \\
\text { CS + } \\
0.5 \% \\
\text { TPP + } \\
0.5 \% \\
\text { Sodium } \\
\text { Citrate } \\
\end{array}$ & $\begin{array}{c}0.5 \% \\
\text { CS + } \\
0.5 \% \\
\text { TPP + } 5 \\
\% \\
\text { GPTMS } \\
\text { Before } \\
\end{array}$ & $\begin{array}{c}0.5 \% \\
\text { CS + } \\
0.5 \% \\
\text { TPP + } 5 \\
\% \\
\text { GPTMS } \\
\text { After } \\
\end{array}$ & $\begin{array}{c}0.5 \% \\
\mathrm{CS}+0.5 \\
\% \text { TPP } \\
(\mathrm{pH} 9)+ \\
0.5 \mathrm{mM} \\
\text { genipin } \\
\text { Before } \\
\end{array}$ & $\begin{array}{c}0.5 \% \\
\text { CS + 0.5 } \\
\% \text { TPP } \\
(\mathrm{pH} \mathrm{9)+} \\
0.5 \mathrm{mM} \\
\text { genipin } \\
\text { After } \\
\end{array}$ & $\begin{array}{c}0.5 \% \\
\text { CS + 0.5 } \\
\% \text { TPP } \\
(\mathrm{pH} 4.5) \\
+0.5 \\
\text { mM } \\
\text { genipin } \\
\text { Before } \\
\end{array}$ & $\begin{array}{c}0.5 \% \mathrm{CS} \\
+0.5 \% \\
\mathrm{TPP}(\mathrm{pH} \\
4.5)+0.5 \\
\text { mM } \\
\text { genipin } \\
\text { After }\end{array}$ \\
\hline $\begin{array}{c}\text { Original Size } \\
\text { Before } \\
\text { Centrifugation }\end{array}$ & 503.9 & 451.7 & 394.4 & 322.3 & 372.4 & 285.3 & 468.7 & 299.1 \\
\hline Supernatant & N/A & 202.3 & 188.5 & 121.6 & 181.9 & 121.9 & 368.4 & 92.7 \\
\hline D.I. Water & 871.48 & 2526.3 & 707.7 & 621 & 708.9 & 459.3 & 722.4 & 595.8 \\
\hline pH 7 & 4563.4 & 2013.3 & 1293.2 & 2496.3 & 3124.7 & 2279.3 & 600.75 & 949.45 \\
\hline pH 5 & 16912.8 & 2316.7 & 3119.7 & 4381.3 & 2590 & 3606.3 & 2709 & 5428 \\
\hline pH 2 & 360.46 & 426.4 & 362.7 & 340.1 & 431.8 & 332.3 & N/A & N/A \\
\hline \multicolumn{9}{|c|}{ Hour 24} \\
\hline D.I. Water & 702.6 & 1798.7 & 604.3 & 298.8 & 599.3 & 392.4 & 605.15 & 453.85 \\
\hline $\mathrm{pH} 7$ & 14738.6 & 1972.3 & 2756 & 1055.1 & 2431.3 & 5938.3 & 1065.35 & 2808.85 \\
\hline pH 5 & 3934.8 & 1265.7 & 2660.3 & 1123.7 & 1806 & 5247.3 & 5254.86 & 4323.3 \\
\hline $\mathrm{pH} 2$ & 315.8 & 369.6 & 292 & 371.9 & 343.3 & 227.4 & N/A & N/A \\
\hline
\end{tabular}

\section{Heppe Medical Chitosan Crosslinked With Genipin}

For the Heppe Medical chitosan, the effects of genipin both on initial size and centrifugation needed to be study. Centrifugation was important as genipin was added to increase the mechanical properties of the particles. However, its effects were different, as compared to the LMW CS crosslinked with genipin. In fact the data showed that the genipin did not contribute any significant mechanical strengthening properties to the CS particles as seen in Figure 29 below, even when the $\mathrm{pH}$ of the TPP solution was changed. Therefore, the addition of genipin was no longer needed in the Heppe Medical chitosan at the new CS and TPP concentrations. 


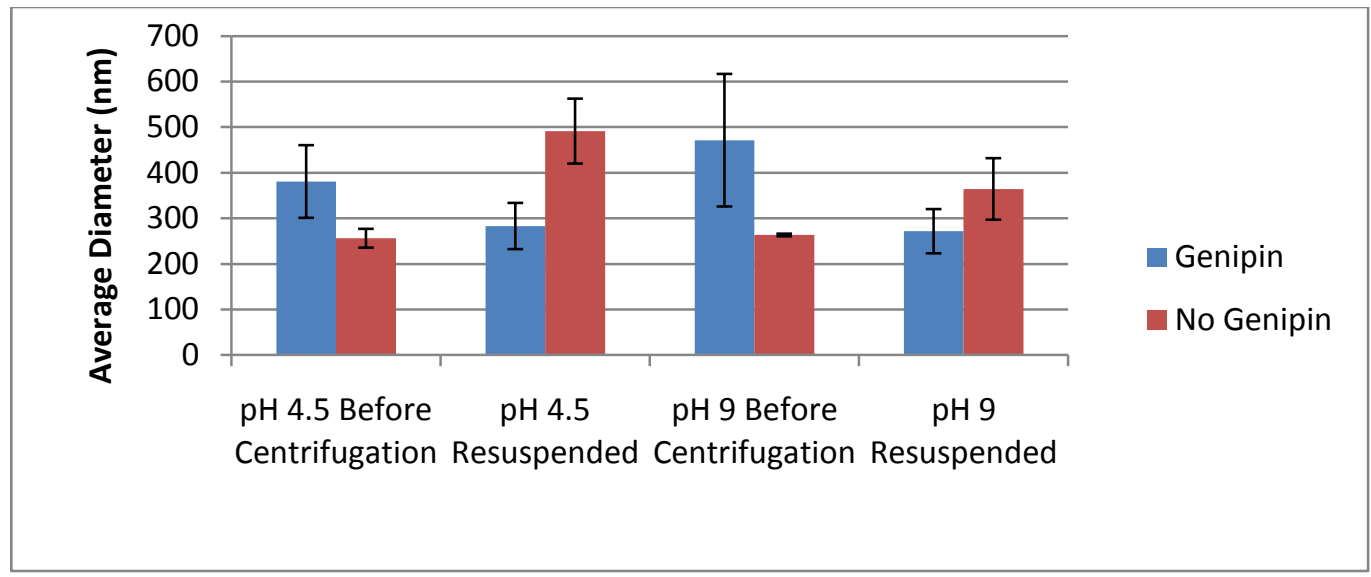

Figure 29 - Heppe Medical Chitosan Particles Crosslinked with Genipin Study

\section{Protein Delivery}

\section{I. $\quad$ BSA Encapsulation in Chitosan Particles}

To determine how much protein is encapsulated within the LMW CS particles, the encapsulation efficiency was calculated for various starting concentrations of BSA. The amount encapsulated was determined by the amount of BSA remaining in the formulation buffer after CS particle formation. This concentration was determined spectroscopically (@280 nm) or using a protein quantification assay. It can be seen in Figure 30 that $0.25 \mathrm{mg} / \mathrm{mL}$ BSA had the highest encapsulation efficiency of $61.1 \pm 35.4$ $\%$. However, a paired t-test showed no statistical difference between any of the BSA concentrations. Hence, there appears to be no trend of BSA concentration vs. encapsulation efficiency within this range. 


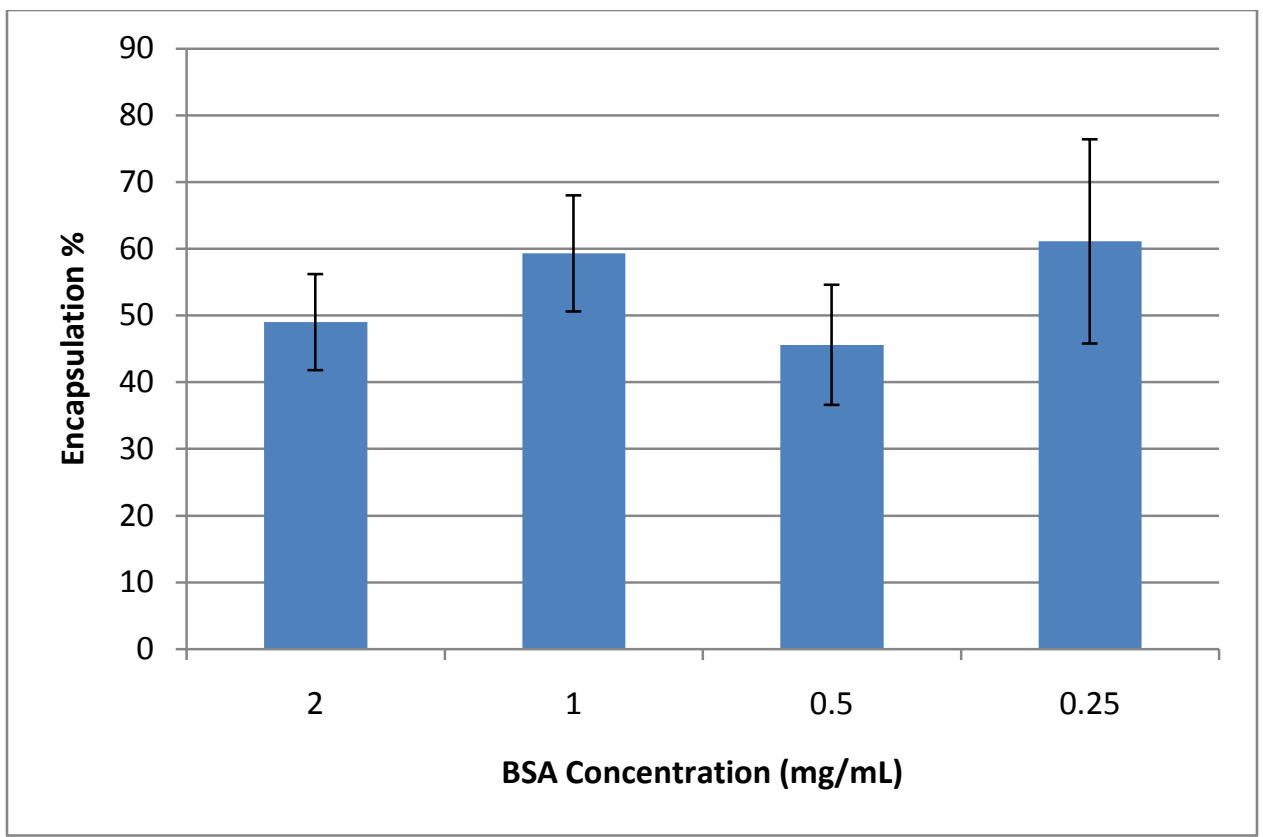

Figure 30 - BSA Encapsulation Efficiency Performed Spectroscopically

A BCA protein quantification assay was also used for encapsulation efficiency

due to its increased sensitivity. The data shows that the encapsulation efficiencies were a lot higher, with $0.5 \mathrm{mg} / \mathrm{mL}$ encapsulated BSA exhibiting the highest rate of encapsulation efficiency as seen in Figure 31. However, again there was no statistical difference between the BSA encapsulated concentrations. It is also important to note that $2 \mathrm{mg} / \mathrm{mL}$ encapsulated BSA was not included in this analysis due to its high signal saturating the plate reader. It is hypothesized that the higher encapsulation efficiencies could be due to the higher sensitivity of the BCA assay. When comparing the encapsulation efficiencies of BSA to NAGase they were found to be similar, equaling approximately $90 \%$. 


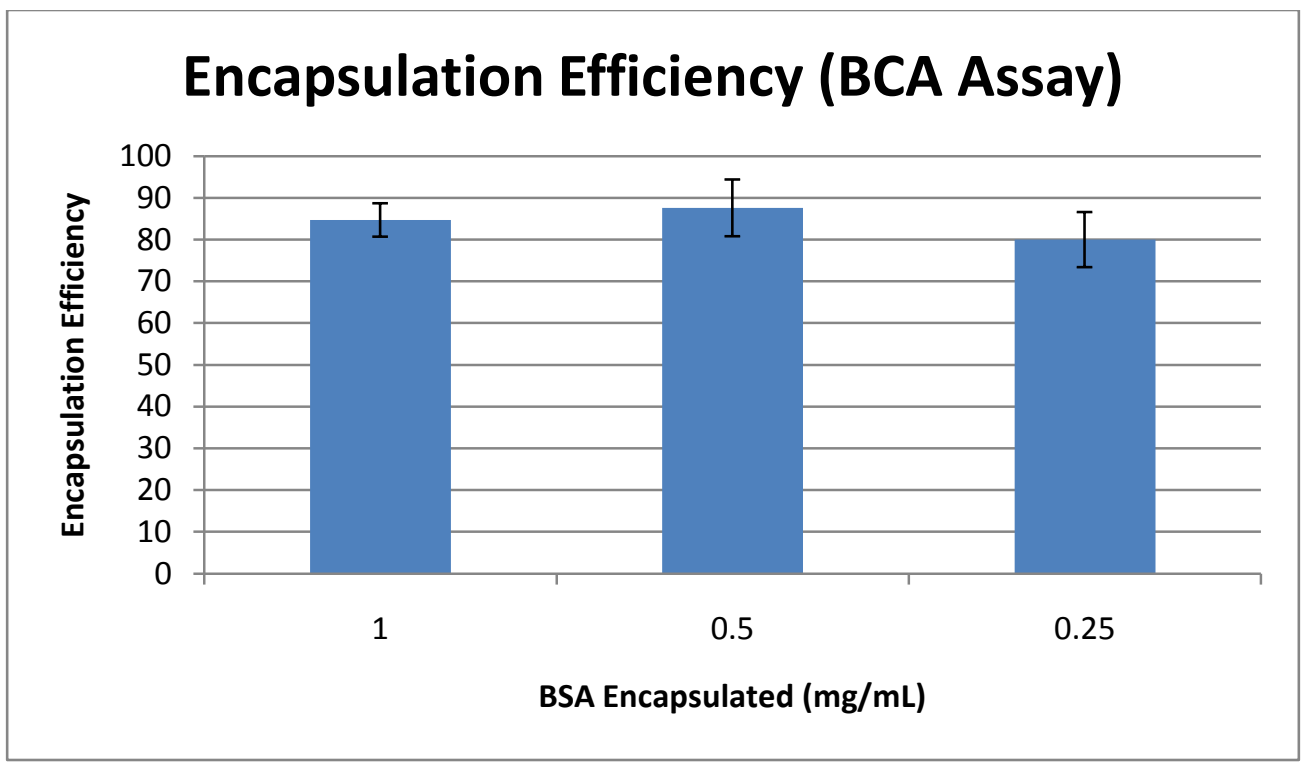

Figure 31 - BSA Encapsulation Efficiency Study Performed Via BCA Assay

In order to visualize protein encapsulated within the CS particles, confocal microscopy was used to image BSA conjugated to FITC within the nanoparticles formed with HMW CS. It can be seen in the images (Figures 32 and 33) that BSA was encapsulated within the CS particles using either method. However, it is also important to note that fibers were observed; however, they did not appear to be encapsulating BSA. 


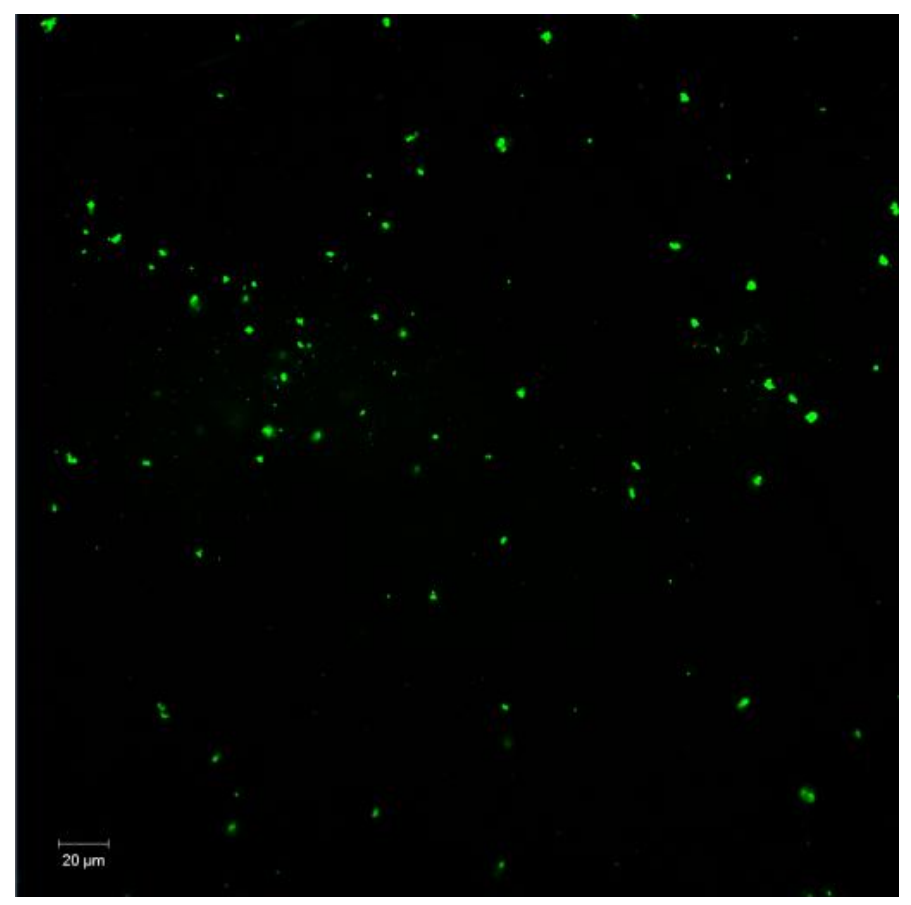

Figure 32 - Confocal Image Encapsulating BSA After Chitosan Particle Formation

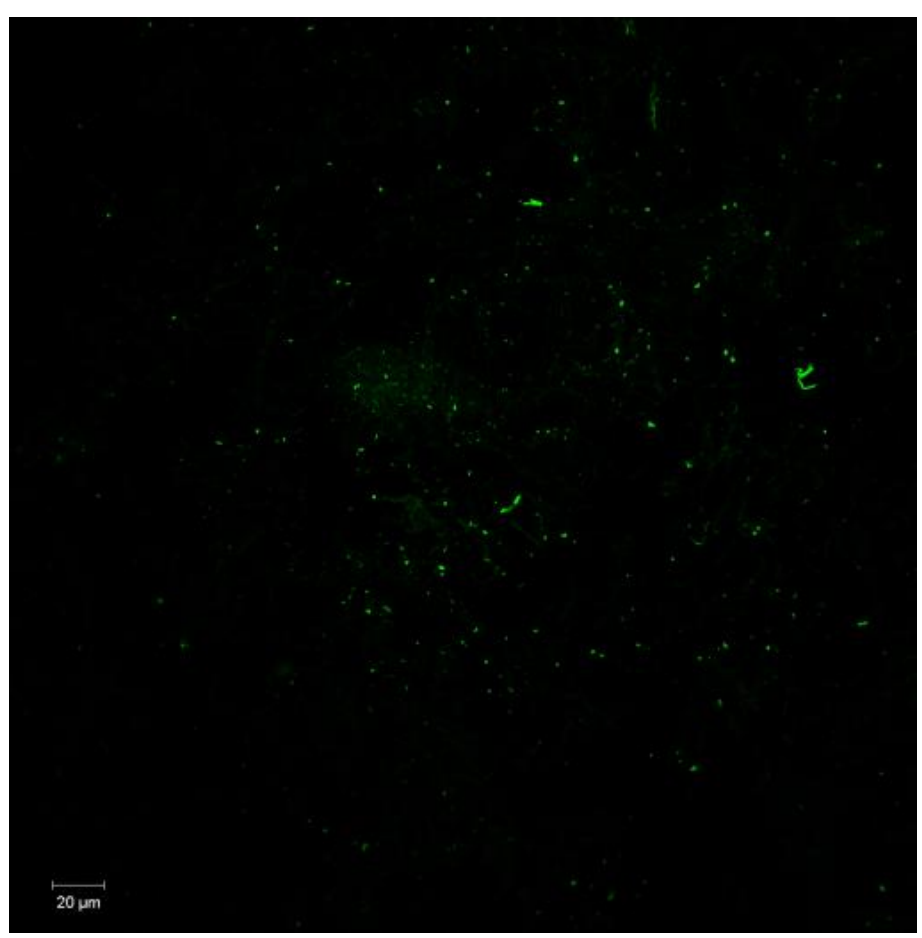

Figure 33 - Confocal Image Encapsulating BSA Before Chitosan Particle Formation 


\section{BSA Release From Chitosan Particles}

a. Low Molecular Weight Chitosan Particles. In order to understand the drug release from $\mathrm{pH}$ dependent CS particles, a swelling study was performed, which encapsulated BSA. Initially, CS particles encapsulating BSA were smaller than the CS particles not encapsulating BSA. They all appear to follow the same trend of having a maximum release at hour 1 (Figure 34 and 35). However, the CS particles encapsulating BSA in PBS ( $\mathrm{pH} 7$ ) did have a steady release from hour 1 to hour 4; instead, of a swelling rate decline. However, it is difficult to confirm that it is not following the same trend due to the large standard deviation. Comparing the data without BSA to the previous study, shows similar hour 1 swelling sizes but this study has less of a pronounced increase at hour 1. Overall, this data shows a greater swelling of CS particles in $\mathrm{pH}$ 5. This is desired; in order, to have a burst release of the NAGase into a pH 5 environment where it has optimal activity.

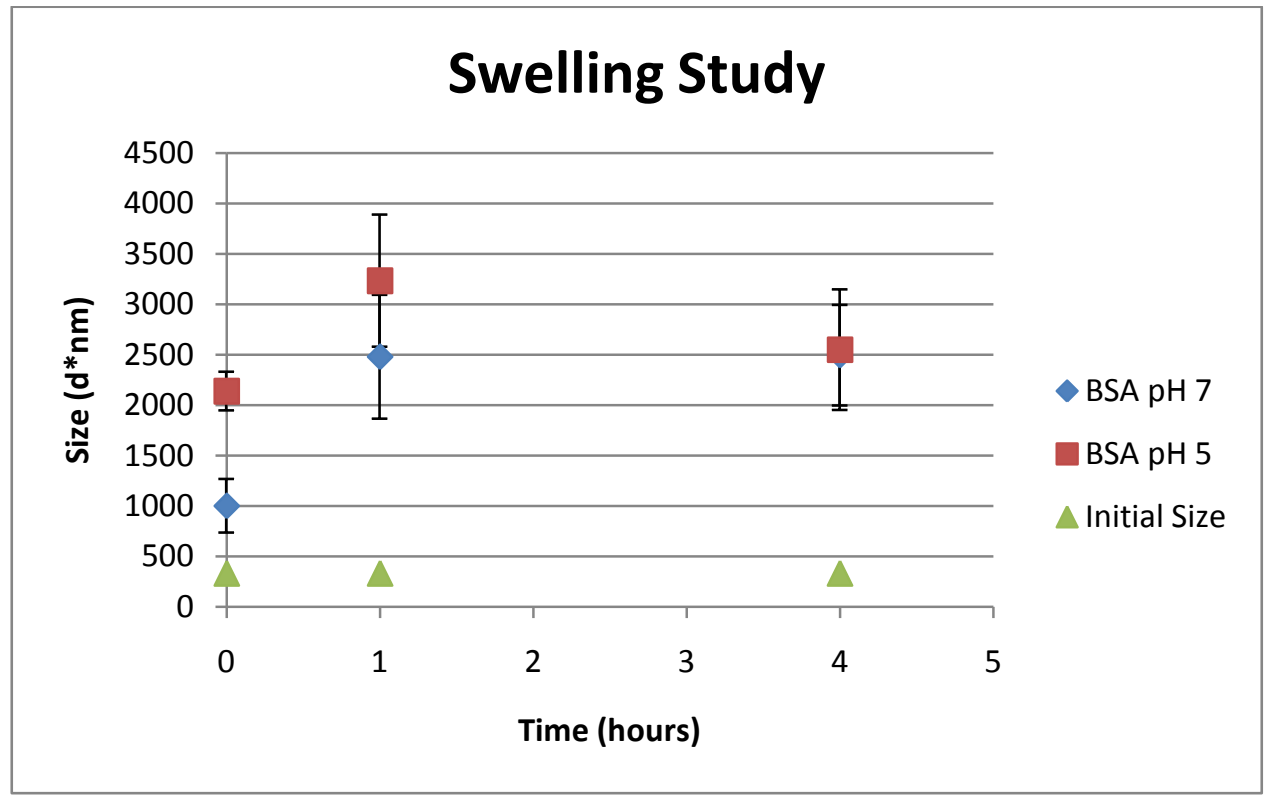

Figure 34 - Swelling Study of Chitosan Particles Encapsulating BSA 


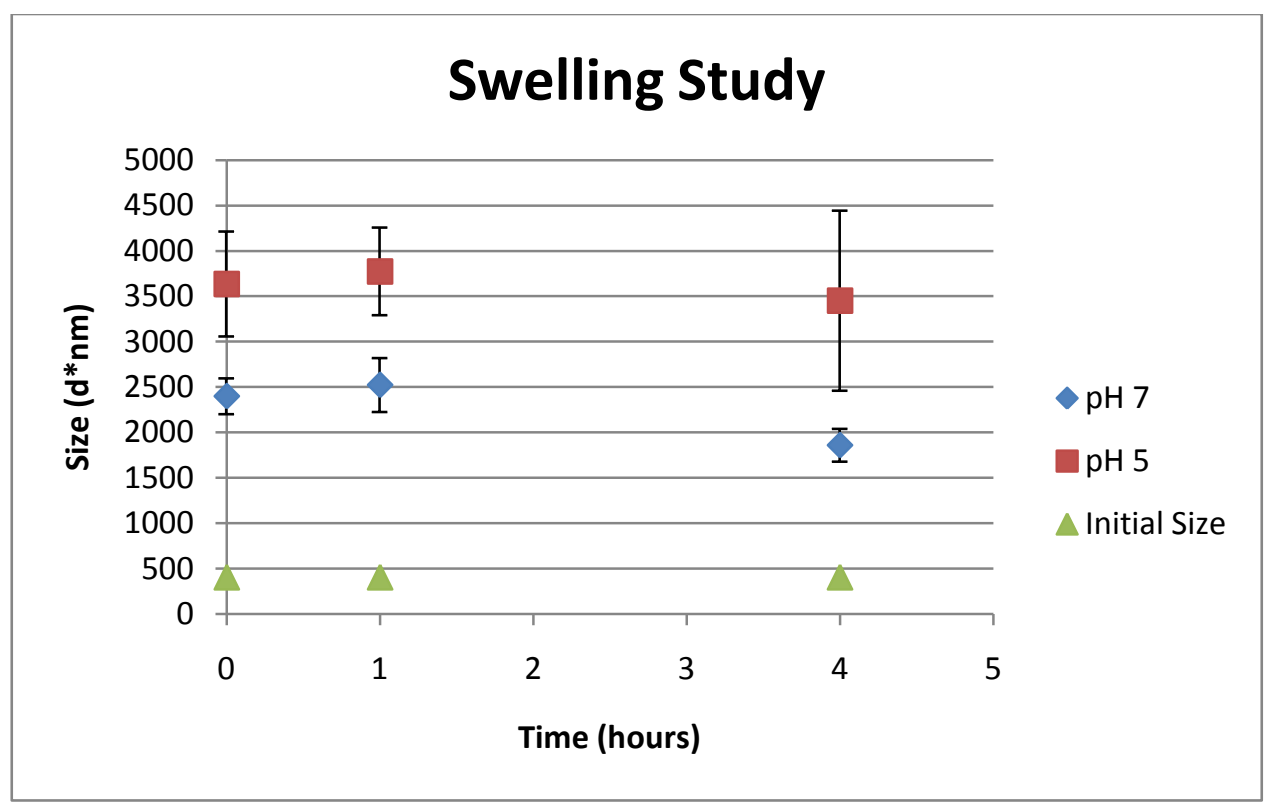

Figure 35 - Swelling Study of Chitosan Particles Not Encapsulating BSA

The release of BSA from LMW CS particles is reported in Figure 36. It is important to note that $1 \mathrm{mg} / \mathrm{mL}$ BSA was dissolved in the CS solution before CS particle formation. In this formulation, BSA release was not detectable until 24 hours; however, given the large standard deviation the statement may be false. In addition, there was no difference seen between the different $\mathrm{pH}$ environments. The delayed release is most likely due to the genipin crosslinking the BSA instead of just the chitosan. From this study it was determined that the encapsulation method of the BSA needed to be further explored; as well as, a more optimal measurement system that is not as affected by chitosan. Therefore, BSA will be encapsulated after CS particle formation if using genipin and a fluorometer will be used to measure BSA concentration. 


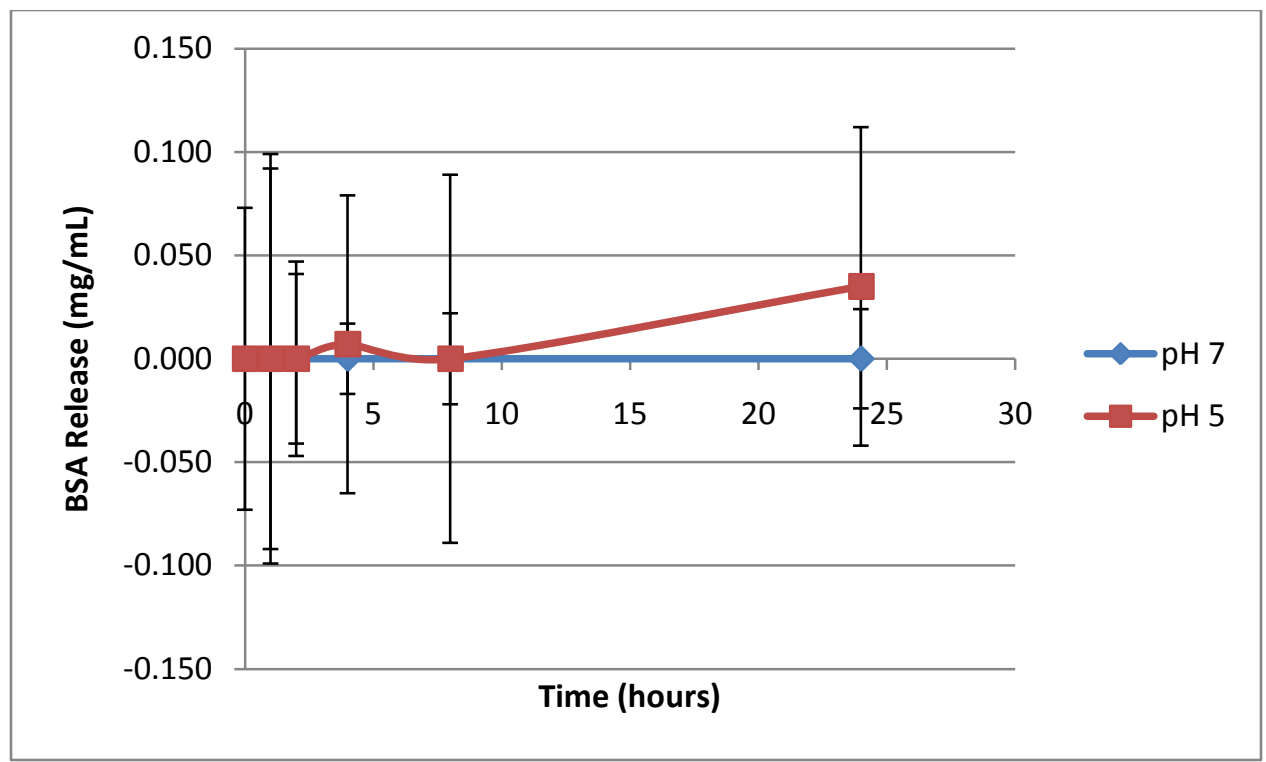

Figure 36 - Release Study of Chitosan Particles Measured Spectroscopically

In the next release study, BSA conjugated with FITC was encapsulated after CS particle formation at a concentration of $0.25 \mathrm{mg} / \mathrm{mL}$. The encapsulation efficiency was approximately $79.3 \pm 4 \%$. The study showed a positive release for every time point but the BSA release rate was extremely low compared to the encapsulation efficiency (Figure 37). Also the release profiles were extremely different for both buffers and do not follow the swelling trends. There is also no clear distinction in which buffer showed a higher BSA release rate. Lastly, with the BSA being encapsulated afterwards there is no burst release and the release rate appears to be reaching 0 within 8 hours. 


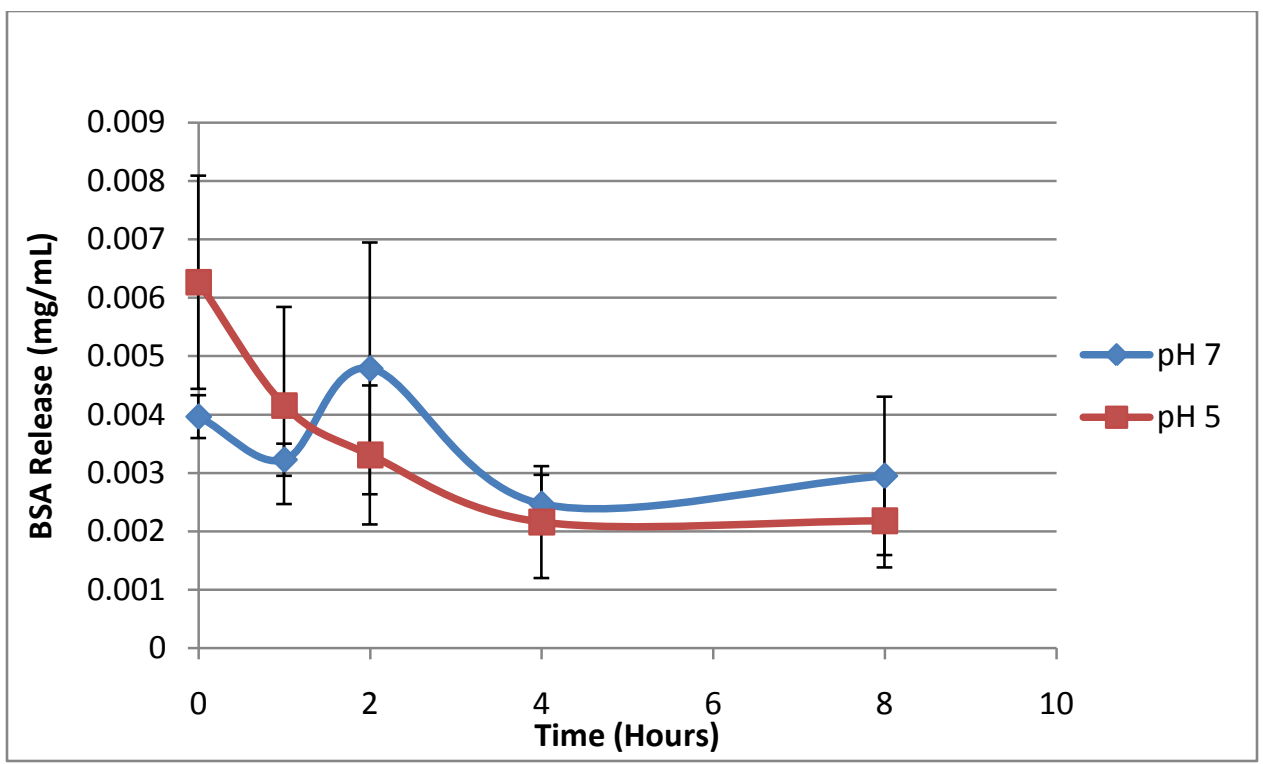

Figure 37 - Release Study of Non-Stirred BSA Measured by Fluorometer

The next release study was similar to the previous study; however, the sample was continuously stirred at room temperature and only $0.125 \mathrm{mg} / \mathrm{mL}$ BSA was encapsulated. The encapsulation efficiency was $37.1 \pm 18.1 \%$, meaning $0.046 \mathrm{mg} / \mathrm{mL}$ was encapsulated. When looking at hour 0 (Figure 38) approximately $4 \%$ of the BSA that was encapsulated was released. This is most likely just the BSA loosely encapsulated on the surface being removed for measurement. After hour 0 there appears to be no release in sodium citrate buffer while BSA release in $\mathrm{pH} 7$ remains constant. 


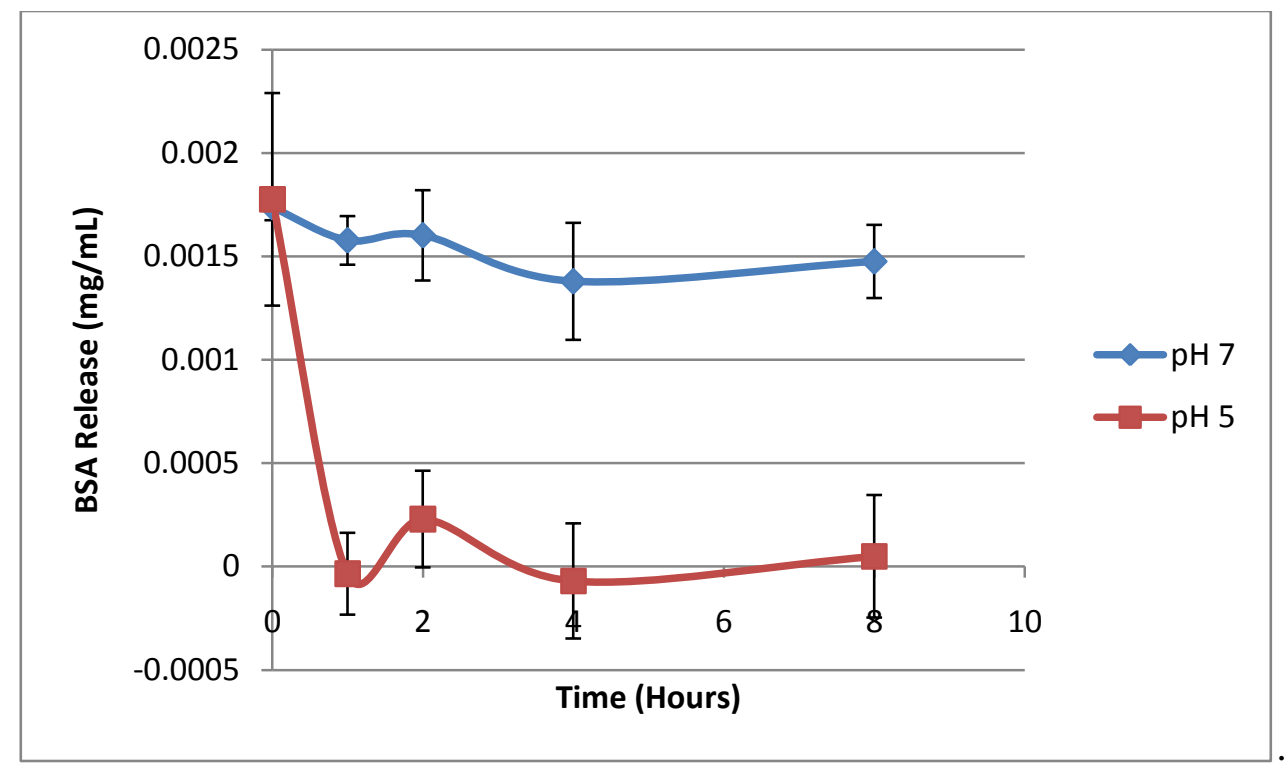

Figure 38 - Release Study of Stirred BSA Measured by Fluorometer

Compared to the first fluorometer study that was not stirred the BSA release was approximately $30 \%$ lower. This was expected due to the difficulty of establishing a standard curve of BSA+FITC. Therefore, more studies must be done to optimize BSA release but what is important is that both exhibited a burst release in a $\mathrm{pH} 5$ buffer. This is important as it relates to a specific aim of the project, which is the CS particles provide a burst in a pH 5 environment so that NAGase is within its optimal activity range.

b. Heppe Medical Chitosan Particles. The next swelling study was performed using Heppe Medical Chitosan. Figure 39 shows a significant increase in CS particle diameters in sodium citrate buffer $(\mathrm{pH} 5)$ at hour 0 when compared to $\mathrm{pH} 7$. The diameter of the CS particles in sodium citrate buffer than drastically decreases to match the CS particles suspended in PBS ( $\mathrm{pH} 7)$. It was also observed that the encapsulation method of the enzyme, whether it is before or after CS particle formation, does not affect the swelling size of the CS particles. As time increases it is expected, based on previous results (data not shown) that the CS particles will remain the same size. Lastly, the 
swelling behavior at $\mathrm{pH} 5$ is desired, since an increase in CS particle diameter should correlate to a burst release of the NAGase. This is again desired because the main release will occur within the target that is the biofilm and in an environment at which NAGase has optimal activity.

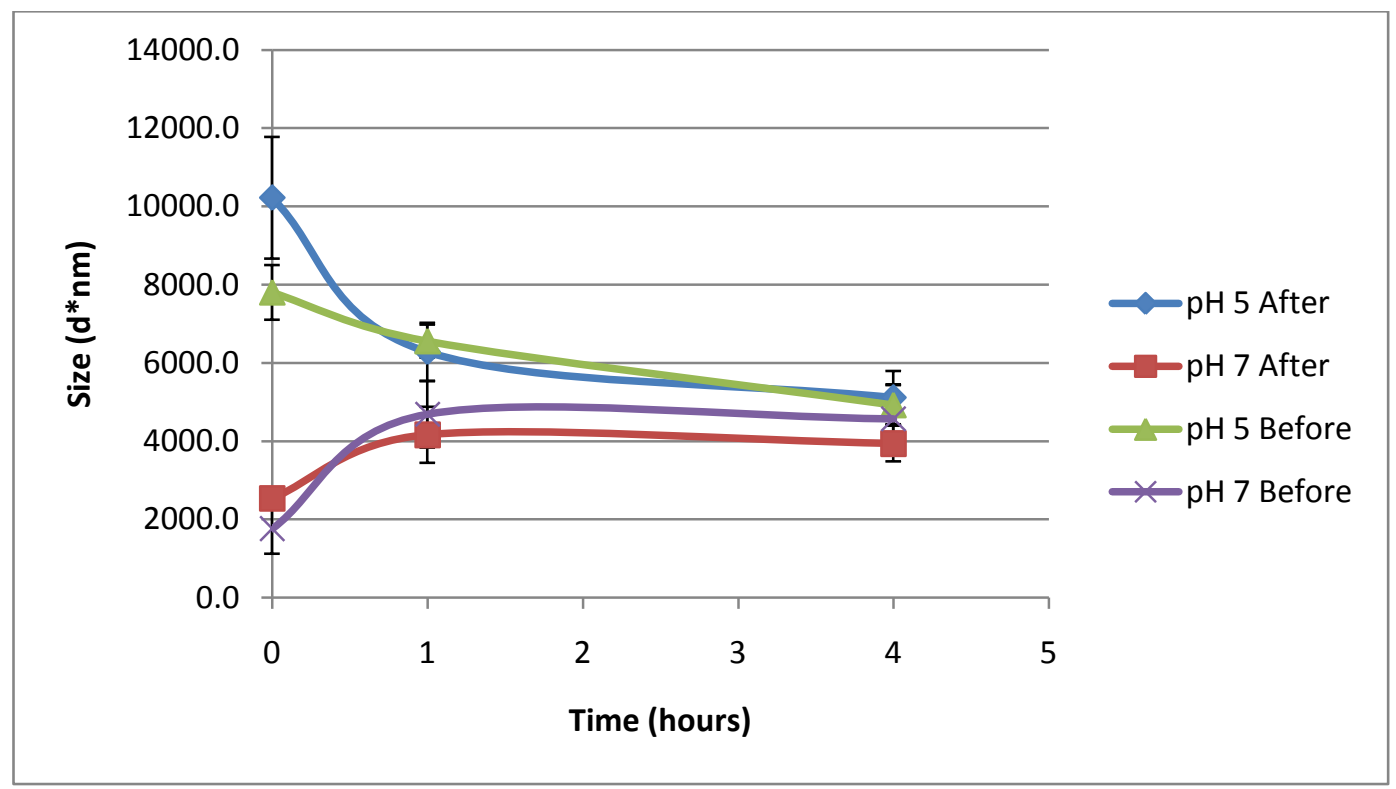

Figure 39 - Heppe Medical Chitosan Particle Swelling Study

For release of BSA from Heppe Medical Chitosan particles a BCA assay was completed; however, due to the molarity of the sodium citrate buffer it had a negative reaction with the kit (data not shown). Therefore, future studies could reduce the molarity of the sodium citrate to levels the BCA assay kit can handle or find a new $\mathrm{pH} 5$ buffer system. It is important to note that a fluorometer release study was not pursued to the difficultly of establishing a standard curve.

\section{NAGase Release from CS Particles}

In the NAGase release study, the encapsulation efficiency was calculated to be $88.89 \pm .36 \%$ but only $1 \%$ of the NAGase was released within 8 hours. While the 
therapeutic dose to degrade the biofilm is not yet established it is hypothesized that this amount of release it too low. The graph also does not correlate to the high concentration burst release of NAGase needed. The study also showed higher release at hour 0 followed by a decrease in release at each time point until stabilization at hour 4 was established (Figure 40). As expected, due to the swelling studies, the release rate of NAGase is higher for CS particles suspended in sodium citrate buffer ( $\mathrm{pH}$ 5) as compared to those suspended in phosphate buffer saline ( $\mathrm{pH} 7$ ). However, the release profile does not follow the expected trend in that the release starts to decrease after hour 0 . This was not expected because CS encapsulated NAGase particles were immediately resuspended in a buffer and then divided into $100 \mu \mathrm{L}$ for each time sample; therefore, the curve should remain the same as hour 0 or the release should increase overtime. Possible reasons as to why this might occur; include, the protein adsorbing to the plate it is contained in or the NAGase using chitosan as a substrate instead of the 4-Nitrophenyl N-acetyl- $\beta$-Dglucosaminide substrate that is used to determine NAGase activity. However, the important objective of this study was proven in that there was release of the NAGase but then must be optimized in future studies. 


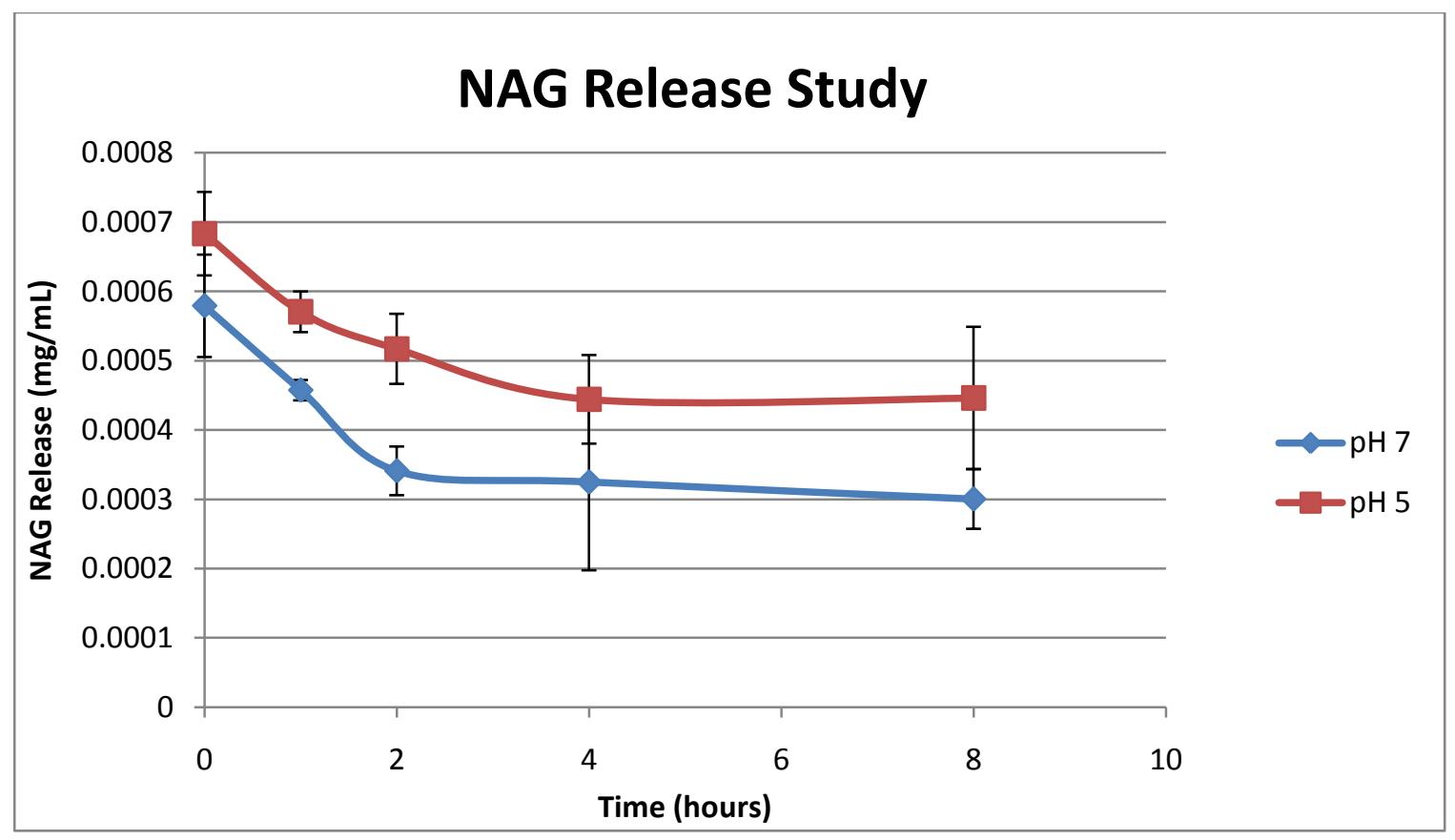

Figure 40 - NAGase Release Study 


\section{CONCLUSIONS}

The research showed that CS nanoparticles can be created through ionic crosslinking with a polyanion, tripolyphosphate. When developing the CS particles as a drug delivery vehicle for a biofilm degrading enzyme, NAGase, it was important that the following parameters be optimized, including: size of CS particles, $\mathrm{pH}$ dependent swelling properties of CS particles, encapsulation efficiency of NAGase and release kinetics of NAGase. These parameters were important to optimize for the function of the CS nanoparticles. Meaning, they must be small enough to fit within the voids and microchannels of the biofilm. They must also exhibit a $\mathrm{pH}$ controlled release of NAGase that is activated by the lower $\mathrm{pH}$ waste pockets of the biofilm. Finally, NAGase release must be established so that it can start to degrade biofilms.

For the CS particles to fit its function, the following parameters were optimized through design of experiments that looked at the concentration of CS and TPP solutions used during particle fabrication, the molecular weight of chitosan used, deacetylation \%, $\mathrm{pH}$ of TPP solution, etc. Another measurement conducted was the zeta potential of the CS particles, which showed they exhibit a positive charge, reaffirming its mucoadhesive properties. The best separation method was found to be high speed centrifugation. The times and speeds were later optimized to achieve the highest CS particle efficiency 
return. However, it was shown through high speed centrifugation that the CS particles have low mechanical strength. Therefore, studies examining different crosslinkers were conducted, such as genipin. Several different imagining techniques were also done to confirm that the CS particles were approximately $200-400 \mathrm{~nm}$ in diameter and were spherical in shape with a small polydistribution. As mentioned earlier, the $\mathrm{pH}$ dependence of the CS particles was established but the sensitivity at $\mathrm{pH} 5$ and $\mathrm{pH} 7$ differed little. Encapsulation was also proven via confocal microscopy and assays with both a model protein BSA and NAGase. Finally, release of BSA and NAGase was shown at both pH 5 and $\mathrm{pH}$ 7. In conclusion, the concept for developing CS nanoparticles to deliver a biofilm degrading enzyme, NAGase, was established; however, future work to better optimize and prove its efficacy still need to be determined. 


\section{RECOMMENDATIONS}

While the concept to develop CS particles encapsulating NAGase for biofilm degradation was proven there are still several parameters that must be optimized. These parameters; include, increasing mechanical properties of the CS particles, better tailoring $\mathrm{pH}$-dependent swelling properties for targeted release, and showing higher release of NAGase. In addition, better imagining is also needed; in order, to view the size, shape and morphology of the CS particles. Finally, release of the NAGase from CS particles must also be proven to cause biofilm degradation. It is the ultimate goal that this drug delivery system can be used alongside an antibiotic releasing silk catheter coating.

The first parameter that needs to be better optimized is the mechanical strength of

the CS particles. There was some work into this area through the addition of crosslinkers, such as sodium citrate, GTPMS and genipin, with genipin showing the best results. Unfortunately, due to time the genipin coating was never fully optimized for the Heppe Medical Chitosan. Therefore, this path should be continued with particular focus on only crosslinking the outside membrane of the CS particles. With this technique it will be important to reestablish swelling and release profiles with and without the use of the genipin coating. If no improvement in mechanical strength is seen during the 
centrifugation process then moving to a different CS particle fabrication process, such as single emulsion or nanoprecitation might be pursued.

The second parameter that needs to be optimized is the $\mathrm{pH}$-dependent swelling properties of the CS particles. This parameter is critical because the entire drug delivery system is dependent on having a controlled release of the NAGase due to changes in $\mathrm{pH}$ of the biofilm microenvironments. In this study, which used ionic gelation to create CS particles, it was found that the $\mathrm{pH}$ triggered release mechanism was not as specific for $\mathrm{pH}$ 5 as was described in the literature. Meaning, CS particles suspended in $\mathrm{pH} 7$ also showed increased swelling, which quickly reached the same swelling levels as pH 5 within 15 minutes of each other. However, when CS particles were suspended in a $\mathrm{pH} 8$ solution, no swelling occurred. This showed that the CS particles could be controlled via a $\mathrm{pH}$ change but the specific $\mathrm{pH}$ value at which swelling is triggered still needs to be better established. Answering this question is essential because the CS particles need to retain most of their payload until they reach the appropriate target. Methods to improve swelling rates rely heavily on understanding the underlying chemical kinetics. Therefore, attention must be paid to the $\mathrm{pH}$ and concentration of the TPP and CS solution before nanoparticle fabrication; as well as, what is occurring during the ionic gelation process. If this cannot be better controlled, a different CS particle fabrication process might need to be pursued.

The third parameter that needs to be optimized is the release of NAGase from CS nanoparticles. In this paper, it was shown that the encapsulation efficiency of NAGase in CS particles was roughly $90 \%$. However, in a NAGase release study it was shown that only $1 \%$ of the NAGase was released within 8 hours. While the therapeutic index has not 
yet been established for biofilm degradation, the amount released in hour 1 is most likely too low. Therefore, a better understanding of why the release is low needs to be determined. Possible answers; include NAGase absorbing to the plate during the release study or NAGase using chitosan as the substrate instead of the substrate found within the activity kit. It will be essential to demonstrate a higher release of NAGase from CS particles before studies involving actual biofilms can be completed.

Due to a combination of factors CS particles were often difficult to image. The main reason was due to the polymeric nature of CS particles, meaning there were fewer to no electrons to excite. Different methods to improve this, such as gold sputter coating, phosphotungstic staining and copper TEM grids were also tried with no results. Another reason for the difficultly in imagining was because the CS particles had to be dried before first, which caused clumping and crystallization of the sample to occur. Methods such as rotary evaporation, vacuum drying and critical flash dryer were tried to reduce the drying effects with no results. Another problem resulted from using lower concentrations of CS and TPP solutions during particle fabrication because they showed a lower CS particle fabrication efficiency. This made it difficult to image individual particles via methods like AFM even when centrifugation was done. Lastly, problems with the resolution of the equipment, especially the SEMs, also occurred due to the small size of the CS particles. One method that provides promise for future SEM and TEM imagining is graphene support TEM films because they have a lower dynamical interference; thereby, reducing background noise. The second promising technique for future confocal imaging is the use of FluoromountG from Southern Biotech. This product is a thick, viscous substance that 
helps to diminish the photobleaching of the fluorescence's while helping the CS particles to better retain their structure during the drying process.

Lastly, it is important to show that the CS particles encapsulating NAGase degrade biofilms through a $\mathrm{pH}$ dependent release. Once proven, the therapeutic dosage must be established. Once characterization of drug delivery system is completed within the in-vitro model, it will need to be joined to antibiotic containing silk film. The in-vitro work must then be repeated again to prove that there is a decrease in antibiotic resistance.

In conclusion, this paper proves that the initial design is possible for this particular application. It is also important to note that through the course of research a lot more questions were raised that still need to be answered; in order, to optimize and prove the efficacy of the design. However, by answering all the questions mentioned above a better and more tailored drug delivery vehicle can be created. 


\section{LIST OF REFERENCES}

Agnihotru, A. S., Mallikarjuna, N. N., \& Aminabhavi, M. T. (2004). Recent advances on chitosan-based micro- and nanoparticle in dryg delivery. Journal of Controlled Release , $5-28$.

Al-fattani, M. A., \& Douglas, J. L. (2006). Biofilm matrix of CAndida albicans and Candida tropicalis: chemical composition and role in drug resistance. Journal of Medical Microbiology, 999-1008.

Berger, J., Reist, M., Mayer, J. M., Felt, O., Peppas, N. A., \& Gurny, R. (2004). Structure and interactions in covalently and ionically crosslinked chitosan hydrogels for biomedical applications. European Journal of Pharmaceutics and Biopharmaceutics , 19-34.

Bodmeier, R., Chen, H., \& Paeratakul, O. (1989). A novel approach to the delivery of microparticles or nanoparticles. Pharmaceutical Research , 413-417. Calvo, P., Remunan, C., J.L.V., J., \& Alonso, M. J. (1997). Novel hydrophilic chitosanpolyethylene oxide nanoparticles as protein carriers. Journal of Applied Polymer Science , 125-132.

Cicalini, S., Palmieri, F., \& Petrosillo, N. (2004). Clinical review: New technologies for prevention of intravascular catheter-related infections. Critical Care, 157-162. 
Donlan, R. M. (2001). Biofilms and device-associated infections. Emerging Infectious Diseases, 277-81.

Drury, W. J., Stewart, P. S., \& Characklis, W. G. (1993). Transport of 1-mum latex particles in pseudomonas aeruginosa biofilms. Biotechnology and Bioengineering , 1117.

Dwyer, A. (2008). Surface-Treated Catheters-A Review. Reducing Tunneled Hemodialysis Catheter Morbidity, 542-546.

Gan, Q., Wang, T., Cochrane, C., \& McCarron, P. (2005). Modulation of surface charge, particle size and morphological properties of chitosan-TPP nanoparticles intended for gene delivery. Colloids and Surfaces, 65-73.

Gan, Q., Wang, T., Cochrane, C., \& McCarron, P. (2005). Modulation of surface charge, particle size and morphological properties of chitosan-TPP nanoparticles intended for gene delivery. Colloids and Surfaces, 65-73.

Goede M.D., M. R., \& Coopersmith M.D., C. M. (2009). Catheter-Related Bloodstream Infection. Surgical Clinical North America , 463-74.

Karchmer, A. W. (2009). Bloodstream infections: the problem and the challenge. International Journal of Antimicrobial Agents , 52-54.

Ko, J. A., Park, H. J., Hwang, S. J., Park, J. S., \& Lee, J. S. (2002). Preparation and characterization of chitosan microparticles intended for controlled drug delivery. International Journal of Pharmaceutics , 165-174.

Li, S. C., \& Li, Y. T. (1970). Studies on the glycosidases of jack bean mean. 3. Crystallization and properties of beta-N-acetylhexosaminidase. Journal of Biological Chemistry, 5153-60. 
Liu, X. F., Guan, Y. L., Yang, D. Z., Li, Z., \& Yao, K. D. (2000). Antibacterial action of chitosan and carboxymethylated chitosan. Journal of Applied Polymer Science, 13241335.

Logghe, C., Van Ossel, C., D'Hoore, W., Ezzedine, H., Wauters, G., \& Haxhe, J. J. (1997). Evaluation of chlorohexidine and silver sulfadiazine impreganated central venous catheters for the prevention of bloodstream infection in leukaemia patients : a randomized controlled trial. Journal of Hospital Infection , 145-156.

Mack, D., Fischer, W., Krokotsch, A., Leopold, K., Hartmann, R., Egge, H., et al. (1996). The intercellular adhesin involved in biofilm accumulation of Staphylococcus epidermidis is a linear beta-1,6-linked glucosaminoglycan: purification and structural analysis. Journal of Bacteriology , 175-183.

Mack, D., Fisher, W., Krokotsh, A., Leopold, K., Hartmann, R., Egge, H., et al. (1996). The intercellular adhesin involved in biofilm accumulation of Staphylococcus epidermidis is a linear beta-1,6-linked glucosaminoglycan: purification and strucutal analysis. Journal of Bacteriology , 175-183.

Maira-Litran, T., Kropec, A., Abeygunawardana, C., Joyce, J., Mark Iii, G., Goldmann, D. A., et al. (2002). Immunochemical Properties of the Staphylococcal Poly-NAcetylglucosamine Surface Polysaccharide. Infection and Immunity , 4433-4440. Marciante, K. D., Veenstra, D. L., Lipsky, B. A., \& Saint, S. (2003). Which antimicrobial impregnated central should we use? Modeling the costs and outcomes of antimicrobial catheter use. American Journal of Infection Control , 1-8. 
Mi, F.-L., Sung, H.-W., Shyu, S.-S., Su, C.-C., \& Peng, C.-K. (2003). Synthesis and characterization of biodegradable TPP/genipin co-crosslinked chitosan gel beads. Polymer , 6521-6530.

O'Grady M.D., N. P., Alexander, M., Dellinger M.D., E. P., Gerberding M.D., M. J., Heard M.D., S. O., Maki M.D., D. G., et al. (2002, August 9). Guidelines for the Prevention of Intravascular Catheter-Related Infections. Retrieved January 18, 2010, from Centers for Disease Control and Prevention: http://www.cdc.gov/mmwr/preview/mmwrhtml/rr5110a1.htm Ohya, Y., Shiratani, M., Kobayashi, H., \& Ouchi, T. (1994). Release behavior of 5fluorourasil from chitosan-gel nanospheres immoblilizing 5-fluorouracil coated with polysaccharides and their cell specific cytotoxicity. Pure Applications Chemistry , 629642.

Pan, W. A., Wu, B. B., \& Wu, M. J. (2009). Chitosan nanoparticles crosslinked by glycidoxypropyltrimethoxysilane for $\mathrm{pH}$ triggered release of protein. Chinese Chemical Letters , 79-83.

Prabaharan, M., \& Mano, J. F. (2005). Chitosan-Based Particles as Controlled Drug Delivery Systems. Drug Delivery, 41-57.

Raad, I. I., \& Hanna, H. A. (2002). Intravascular catheter-related infections. New horizons and recent advances. Archives of Internal Medicine, 871-878.

Raad, I., Costerton, W., Sabharwal, U., Sacilowski, M., Anaissie, E., \& Bodey, G. P. (1993). Ultrastructural analysis of indwelling vascular catheters: a quantitative relationship between luminal colonization and duration of placement. Journal of Infectious Diseases , 400-7. 
Shu, X. Z., \& Zhu, K. J. (2002). Controlled drug release properties of ionically crosslinked chitosan beads: the influence of anion structure. International Journal Pharmacology, 217-225.

Stewart, P. S., \& Costerton, J. W. (2001). Antibiotic resistance of bacteria in biofilms. The Lancet , 135-138.

Sung, H. W., Huang, R. N., Huang, L. L., \& Tsai, C. C. (1999). In vitro evaluation of cytotoxicity of a naturally occurring cross-linking reagent for biological tissue fixation. Journal of Biomaterial Science, Polymer Edition , 63-78.

Sutherland, I. W. (2001). The biofil matrix-an immobilized but dynamic microbial environment. Trends in Microbiology, 222-227.

Watzke, H. J., \& Dieschbourg, C. (1994). Novel silica-biopolymer nanacomposites: the silica sol-gel process in biopolymer organogels. Advances in Colloid and Interface Science, 1-14.

Xu, Y., \& Du, Y. (2003). Effect of molecular structure of chitosan on protein delivery properties of chitosan nanoparticles. International Journal of Pharmaceutics , 215-226. 
VITA

Lindsay Strotman received her Bachelor's Degree of Science in Bioengineering with highest honors from the University of Louisville in 2009. Within that time she completed three co op rotations. The first was with Ethicon, Inc. (Johnson and Johnson) in fall 2007 in Somerville, NJ, where she worked as an R\&D co-op in Women's Health and Urology. The second two co-op rotations where completed in summer 2008 and spring 2009 at Ethicon-Endo Surgery, Inc. in Cincinnati, OH. There she again served as an R\&D co-op in both ultrasonic energy and endoscopy. In-between co-op periods she served as a Supplemental Instructor for Linear Algebra. She also worked in the lab of Andrea Gobin, $\mathrm{PhD}$. researching chitosan as a potential drug delivery vehicle for a biofilm degrading enzyme. This lab experience led to writing and receiving funding for an undergraduate research grant; as well as, travel to research conferences. Next, she will be attending the University of Wisconsin in Madison, WI to complete her doctorial studies under David Beebe, PhD. 